\title{
Quantification of the spatial and temporal evolution of stratified shear instabilities at high Reynolds number using quantitative acoustic scattering techniques
}

\author{
by \\ Jonathan Randall Fincke \\ B.S., University of New Hampshire (2011) \\ Submitted in partial fulfillment of the requirements for the degree of \\ Master of Science in Oceanographic Engineering \\ at the \\ MASSACHUSETTS INSTITUTE OF TECHNOLOGY \\ and the \\ WOODS HOLE OCEANOGRAPHIC INSTITUTION
}

February 2015

(C) Jonathan Randall Fincke, MMXV. All rights reserved

The author here by grants to MIT and WHOI permission to reproduce and to distribute publicly

paper and electronic copies of this thesis document in whole or in part in any medium now known or hereafter created.

Author

Department of Mechanical Engineering and Department of Applied Ocean Science and Engineering January 16, 2015

Certified by

Dr. Andone Lavery

Thesis Supervisor

Woods Hole Oceanographic Institution

Certified by

Dr. W. Rockwell Geyer

Thesis Supervisor

Woods Hole Oceanographic Institution

Accepted by

Dr. Henrik Schimdt

Professor of Mechanical and Ocean Engineering

Massachusetts Institute of Technology

Accepted by

Dr. David Hardt

Professor of Mechanical Engineering

Chair, Committee on Graduate Students - Mechanical Engineering

Massachusetts Institute of Technology 


\title{
Quantification of the spatial and temporal evolution of stratified shear instabilities at high Reynolds number using quantitative acoustic scattering techniques
}

\author{
by \\ Jonathan Randall Fincke \\ B.S., University of New Hampshire (2011) \\ Submitted to the Department of Mechanical Engineering and Department of \\ Applied Ocean Science and Engineering on January $16^{\text {th }} 2015$, in partial \\ fulfillment of the requirements for the degree of Masters' of Science in \\ Mechanical Engineering
}

\begin{abstract}
The spatial and temporal evolution of stratified shear instabilities is quantified in a highly stratified and energetic estuary. The measurements are made using high-resolution acoustic backscatter from an array composed of six calibrated broadband transducers connected to a sixchannel high-frequency $(120-600 \mathrm{kHz})$ broadband acoustic backscatter system. The array was mounted on the bottom of the estuary and looking upward. The spatial and temporal evolution of the waves is described in terms of their wavelength, amplitude and turbulent dissipation as a function of space and time. The observed waves reach an arrested growth stage nearly 10 times faster than laboratory and numerical experiments performed at much lower Reynolds number. High turbulent dissipation rates are observed within the braid regions of the waves, consistent with the rapid transition to arrested growth. Further, it appears that the waves do not undergo periodic doubling and do not collapse once their maximum amplitude is reached. Under some conditions long internal waves may provide the perturbation that decreases the gradient Richardson number so as to initiate shear instability. The initial Richardson number for the observed instabilities is likely between 0.1 and 0.2 based on the slope and growth rate of the shear instabilities.

Thesis Supervisor: Andone Lavery Title: Associate Scientist with Tenure Woods Hole Oceanographic Institution

Thesis Supervisor: W. Rockwell Geyer Title: Senior Scientist Woods Hole Oceanographic Institution
\end{abstract}




\section{Dedication}

This thesis is dedicated to my Fiancé, Hannah, my parents, Randall and Nancy, and brothers, Greg and Chris, for their unconditional support and understanding throughout the years preceding and during my pursuit of this degree. I don't know how this would have come to be without them. 


\section{Acknowledgments}

I would like to thank my Advisers Dr. Andone C. Lavery and Dr. W. Rockwell Geyer for their support, patience and advice as I navigated my way through courses, field work and analysis of the data we collected. Their ability to push their students' and themselves to do the highest quality research possible is something I will be forever thankful for. It has been a pleasure working with them both.

Thank you to my MIT and WHOI friends Matt, Sheida, Jen, Thomas, Tom and many others who have made the journey through classes and research so much more fun.

To my good friends Will and Greg, thank you for being there to hang out whenever necessary.

This thesis would not have been possible without support from the Department of Defense (DoD) through the National Defense Science \& Engineering Graduate Fellowship (NDSEG) Program and the Office of Naval Research (through ONR Grant \#N00014-11-10058) 


\section{Contents}

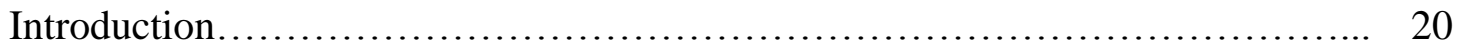

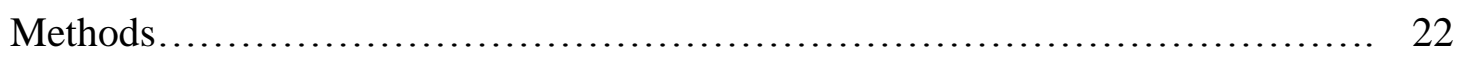

Results......................................................... 31

Discussion...................................................... 41

Conclusions.................................................... 49

Appendix 1: Additional figures.................................... 58

Appendix 2: Additional observations................................. 71

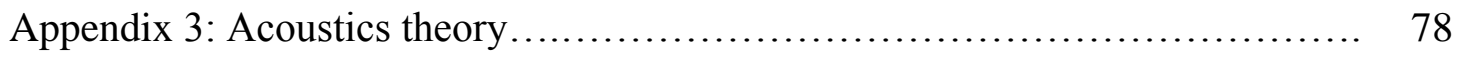

Appendix 4: Stratified flow and turbulence theory $\ldots \ldots \ldots \ldots \ldots \ldots \ldots \ldots \ldots \ldots \ldots \ldots$

Appendix 5: Array design.......................................... 93 


\section{List of Figures}

Figure 1. Google Earth image of the deployment location in the lower Connecticut

River estuary.

Figure 2. Schematic of the bottom-mounted, six-transducer, high-frequency, broadband acoustic backscatter array

Figure 3. Acoustic image from transducer 6 showing a packet of stratified shear instabilities that occurred during the morning ebb tide on December $7^{\text {th }}$

(Observation A).

Figure 4. Acoustic image from transducer 6 during the morning ebb tide on December $7^{\text {th }}$ (Observation A) with the output of the edge detection algorithm marking the packet of shear instabilities

Figure 5. Acoustic images of shear instabilities from all six transducers on the array

Figure 6. Peak-to-peak amplitude of the stratified shear instabilities shown in

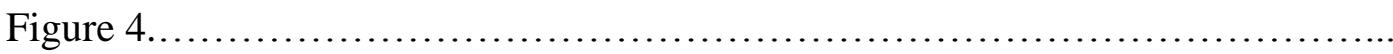

Figure 7. Velocity, $R i$, and density profiles for the stratified shear instability from Observation A (Figure 2). a.) velocity profile obtained from the ADCP on the array, b.) Density profile, inferred from the CTD data. c.) Estimates of $R i$ using the profiles from a.) and b.)

Figure 8. Four echograms showing the growing shear instabilities from

Observations B through E. a-d) Shear instability analyzed from three different days during similar phases of the ebb tide

Figure 9. a.) Amplitude vs. $t \backslash \tau$ for all the stratified shear instabilities from the 
December 2012 experiment in the Connecticut River presented in this paper. b.) same data as in a.) but with all the curves starting at the same time and varying yaxis ranges for each Observation

Figure 10. Spectral classification of the backscattering source. a) Acoustic image from transducer 4 with the black outline illustrating the section of data analyzed. b) Result of the spectral analysis, the spectrum is consistent with backscatter from stratified turbulence, the dashed line is the theoretical slope for volume backscatter from stratified turbulence in the viscous convective sub-range

Figure 11. Data selected from transducer 1 and transducer 6 to make estimates of the change in backscatter strength.

Figure 12. Relationship between wave slope and $R i_{o}$ from Thorpe [1973] and Wroblewski et al. [2007] and references therein.

Figure 13. Acoustic image from transducer 1 on 7 December $2012 \ldots \ldots \ldots \ldots \ldots \ldots \ldots$

Figure 14. $R i$ as function of distance from the trough of an internal wave.

Figure 15. Model predictions from Corcos and Sherman (1976) plotted with the stratified shear instabilities from Observation A showing rough agreement in the time scale of the growth phase

Figure 16. Schematic of the box model used to estimate total energy dissipation....

Figure 1A1. Tidal amplitude with the time of Observations A-E marked with dots.

Figure 2A1. Velocity, $R i$, and density profiles for the stratified shear instabilities from Observation B (Figure 3A1 ............................................ 
Figure 3A1. Acoustic images from all six transducers on the array for Observation

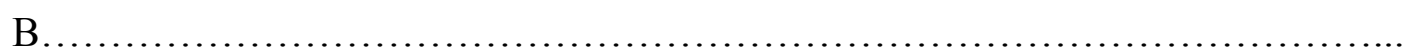

Figure 4A1. Data selected from transducer 1 and transducer 6 to make estimates in the change backscatter strength and spectral classification of the backscattering source. a) The section of data analyzed from transducer one outlined by the black line. b) The section of data analyzed from transducer six outlined by the black line. c) Result of the spectral analysis, the spectrum is consistent with backscatter from stratified turbulence. Transducers three and four were used to create this spectrum.. Figure 5A1. Velocity, $R i$, and density profiles for the stratified shear instabilities from Observation C (Figure 6A1).

Figure 6A1. Acoustic images from all six transducers on the array from Observation C.

Figure 7A1. Data selected from transducer 1and transducer 6 to make estimates in the change backscatter strength and the backscatter spectrum estimated from transducers 3 and 4. a) The section of data analyzed from transducer 1 outlined by the black line. b) The section of data analyzed from transducer 6 outlined by the black line. c) Result of the spectral analysis, the spectrum is consistent with backscatter from stratified turbulence. Transducers 3 and 4 were used to create this spectrum.

Figure 8A1. Velocity, $R i$, and density profiles for the stratified shear instabilities from Observation D (Figure 9A1)

Figure 9A1. Acoustic images from all six transducers on the array from Observation D 
Figure 10A1. Data selected from transducer 1and transducer 6 to make estimates in the change backscatter strength and the backscatter spectrum estimated from transducers 3 and 4. a ) The section of data analyzed from transducer 1 outlined by the black line. b) The section of data analyzed from transducer 6 outlined by the black line. c) Result of the spectral analysis, the spectrum is consistent with backscatter from stratified turbulence. Transducers 3 and 4 were used to create

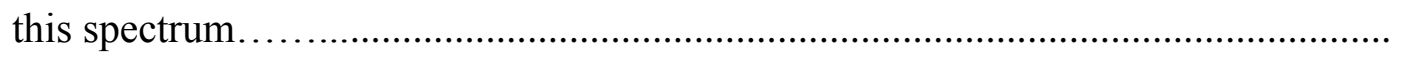

Figure 11A1. Velocity, $R i$, and density profiles for the stratified shear instabilities from Observation E (Figure 12A1) .................................. 66

Figure 12A1. Acoustic images from all six transducers on the array from Observation E........................................................

Figure 13A1. Data selected from transducer 1and transducer 6 to make estimates in the change backscatter strength and the backscatter spectrum estimated from transducers 3 and 4. a) The section of data analyzed from transducer 1 outlined by the black line. b) The section of data analyzed from transducer 6 outlined by the black line. c) Result of the spectral analysis, the spectrum is consistent with backscatter from stratified turbulence. Transducers 2 and 4 were used to create this spectrum.

Figure 14A1. Median alignment through the water column of the backscatter array. The array is typically aligned within 10 degrees of the flow during ebb tides and within 15 degrees during flood tides.

Figure 15A1. a) Array alignment throughout the water column as a function of time and. b) The tidal amplitude during the deployment........................ 
Figure 1A2. Velocity profiles for the along array (along channel) direction during the portion of the deployment where the array was parallel to the flow....

Figure 2A2. Tidal amplitude recorded from the CTD fixed to the array. Note the amplitude of the tidal range varies and cycles with a twelve hour period.

Figure 3A2. Density profiles that were obtained by completing CTD casts for five minutes four times an hour during the experiment

Figure 4A2. Acoustic image from Transducer $4(\mathrm{M})$ at 08:36 on 7 December during the middle of the ebb tide....

Figure 5A2. ADCP time series collected while the array was perpendicular to the flow.

Figure 6A2. a.) Mean volume backscatter in $\mathrm{dB}$ re $1 \mu \mathrm{Pa}$ for a 15 minute section of data estimated from transducers 3 and 4. b) Locations within the water column of scattering consistent with stratified turbulence in the viscous convective subrange.

Figure 7A2. Acoustic image from Observation A.

Figure 1A3. Diagram illustrating what different values of $k a$ look like in regards to an arbitrary inhomogeneity

Figure 2A3. Theoretical volume backscatter strength for selected scattering targets in the ocean

Figure 3A3. a) Chirp signal in the time domain. b.) Discrete time Fourier Transform of the chirp signal (blue) and the pulse compressed chirp signal (blue). c.) pulse compressed chirp signal 
Figure 1A4. A stably stratified and sheared environment illustrating the basic flow characteristics in the Connecticut River......................................

Figure 2A4. A theoretical Batchelor spectrum for a passive tracer such as salinity or temperature in a stratified and turbulent flow............................ 91

Figure 1A5. The high frequency broadband acoustic backscatter array............ 94

Figure 2A5. The high frequency broadband acoustic backscatter array dimensions. 95 


\section{List of Tables}

Table 1. Specifications for the 6 transducer mounted on the array............... 25

Table 2. . Estimates of $R i_{\text {local }}, V_{A D C P}, V_{\text {array }}^{\text {wave }}, h_{\text {max }}$ and $\lambda$ inferred from the

combination of data from the array, ADCP and CTD.................................... 34

Table 3. Shear time scales for each Observation........................... 37

Table 4. Change in $\varepsilon$ from transducer 1 to transducer 6 for Observations A

through E............................................................... 40.

Table 5. Results of $R i_{o}$ calculations from both methods for the five stratified

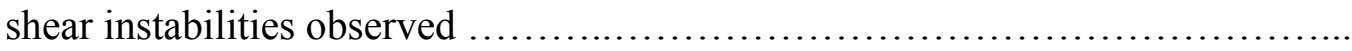




\section{Introduction}

Vertical mixing via stratified shear instability and the resulting stratified turbulence are of central importance to understanding the transport of momentum, passive tracers and heat within the stably stratified ocean and atmosphere [Wunsch and Ferrari, 2004]. Therefore, shear instability has significant implications for vertical exchange processes across a broad range of scales, affecting contaminant dispersal as well as global weather patterns. Shear instabilities occur when the strength of the shear in the flow overcomes the stratification. The balance between shear and stratification is parameterized by the gradient Richardson number $R i=$ $(g / \bar{\rho}) \Delta \rho \Delta z /(\Delta u)^{2}$ where $\mathrm{g}$ is the gravitational constant, $\bar{\rho}$ is the mean density and $\Delta \rho$ is the density difference and $\Delta u$ is the velocity difference across the shear layer of thickness $\Delta z$. In agreement with the theoretical prediction by Miles [1961] stratified shear flow has been shown to be unstable when $R i<1 / 4$ in numerical, laboratory and field experiments [Fernando, 1991; Smyth, 2003; Mashayek and Peltier, 2012; Geyer and Smith, 1987].The spatial and temporal evolution of shear instabilities has been well documented in both numerical and laboratory experiments [Fernando, 1991; Smyth, 2003; Mashayek and Peltier, 2012].

The initial Richardson number, $R i_{o}$ which refers to the $R i$ immediately preceding the growth of a shear instability, is readily documented in laboratory and numerical experiments based on the initially unperturbed stratified shear flow. However in field measurements, the conditions prior to the observation of instabilities usually exhibit significant temporal and spatial variability, such that the estimation of $R i_{o}$ is problematic. Moreover, the spatial scales required to resolve $R i_{o}$ are often smaller than the resolution of the instruments providing stratification and shear measurements. Therefore, obtaining meaningful estimates of $R i_{o}$ and the processes that 
cause it to reach an unstable value is a challenging but important issue that will lead to an improved mechanistic understanding of when and where mixing occurs in natural systems.

A number of theoretical models describing the evolution of shear instabilities have been developed [Miles, 1961; Hazel, 1972; Corcos and Sherman, 1976; Carpenter et al., 2013]. Among these, the Corcos and Sherman [1976] model predicts the high $R e$ two dimensional evolution of shear instability via the final amplitude, growth time and location of the strongest mixing. This model predicts that at high $R e$ shear instabilities arrest their growth faster and the majority of the mixing occurs within the braids.

Many geophysical flows reach a $R e$ much greater than the value attainable in laboratory or numerical experiments. Numerous studies have used acoustic backscatter techniques to image high Re shear instabilities in the field [Seim and Gregg, 1994; Moum at al., 2003; Tedford et al., 2009; Geyer et al., 2010; Lavery et al., 2013]. These studies quantify the occurrence, wavelength and amplitude of shear instability in the field at high Re. Further, Geyer et al. [2010] demonstrated mixing is strongest within the braids of the waves in high $R e$ geophysical flows. However, none of these observations have quantified the evolution, including growth rate, or $R i_{o}$ of individual waves.

This study captures the growth rate and change in turbulence dissipation within the braids of individual waves in the Connecticut River estuary using an array composed of six calibrated broadband transducers connected to a six-channel high-frequency broadband acoustic backscatter system. Unlike previous acoustic observations, which have involved either time series measurements at a single location or spatial shipboard surveys, the downstream spatial separation of transducers on the array employed here allows for quantification of the spatial and temporal evolution of shear instabilities as they advect over the array in the flow direction. 
In addition to quantifying the growth rate of shear instabilities, $R i_{o}$ is inferred using information about the growth rate and two-dimensional structure of the shear instabilities based on previous observations and simulations of [Thorpe, 1973; Smyth, 2003; Fritts et al., 2014]. Insight into the large scale forcing is gained by investigating an acoustic image which captures flow features occurring on time scales longer than the evolution time of shear instabilities. The longer timescale image shows that instability can occur on a pycnocline affected by long internal waves, which could be causing an increase in shear across the pycnocline.

\section{Methods}

Study site

The Connecticut River estuary is a highly stratified and energetic salt-wedge estuary where previous studies have documented the frequent occurrence of shear instabilities (Figure 1) [Geyer et al., 2010; Lavery et al., 2013]. Stratification and shear in the water column is set up by the river outflow over the dense salt wedge as it encroaches up the river estuary. Shear instabilities are generated at the strongly sheared and stratified interface of the salt wedge and fresh river water during ebb and flood tides. Typical turbulent kinetic energy dissipation rates, $\varepsilon\left(\mathrm{m}^{2} / \mathrm{s}^{3}\right)$, in this system can reach $10^{-3} \mathrm{~m}^{2} / \mathrm{s}^{3}$ [Lavery et al., 2013], many orders of magnitude higher than typical oceanic values. At the study site, bed-to-surface differences in velocity and salinity $>1 \mathrm{~m} / \mathrm{s}$ and $25 \mathrm{psu}$, respectively, are observed during the ebb and flood. During the flood the salt-wedge advects up the estuary, resulting in boundary layer turbulence in the salt layer and fresh water outflow above the salt wedge. During the ebb tide the fluid in the salt-wedge is nearly stationary and the freshwater outflow erodes the salt wedge until there is no remaining stratification. 


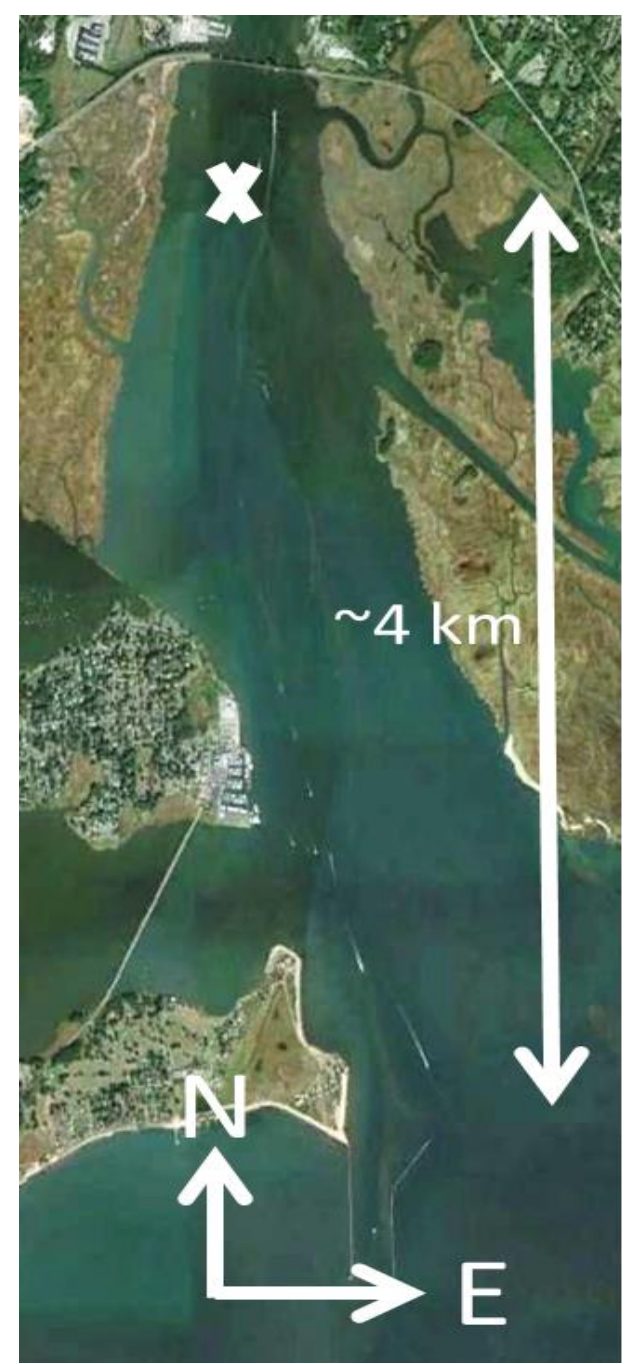

Figure 1. Google Earth image of the deployment location in the lower Connecticut River estuary. The array was deployed at the location of the white $\mathrm{x}$ from 5-8 December 2012. The body of water at the bottom of the figure is Long Island Sound.

\section{Instrumentation}

The suite of instrumentation included an array, consisting of a six-channel calibrated high-frequency broadband acoustic backscatter system with six upward-looking broadband transducers bottom-mounted on a steel frame. Mounted to the frame was an upward-looking 1.2 MHz RDI workhorse acoustic Doppler velocity profiler (ADCP). A RBR conductivity, temperature, and depth (CTD) sensor was deployed by hand from a nearby ship. 


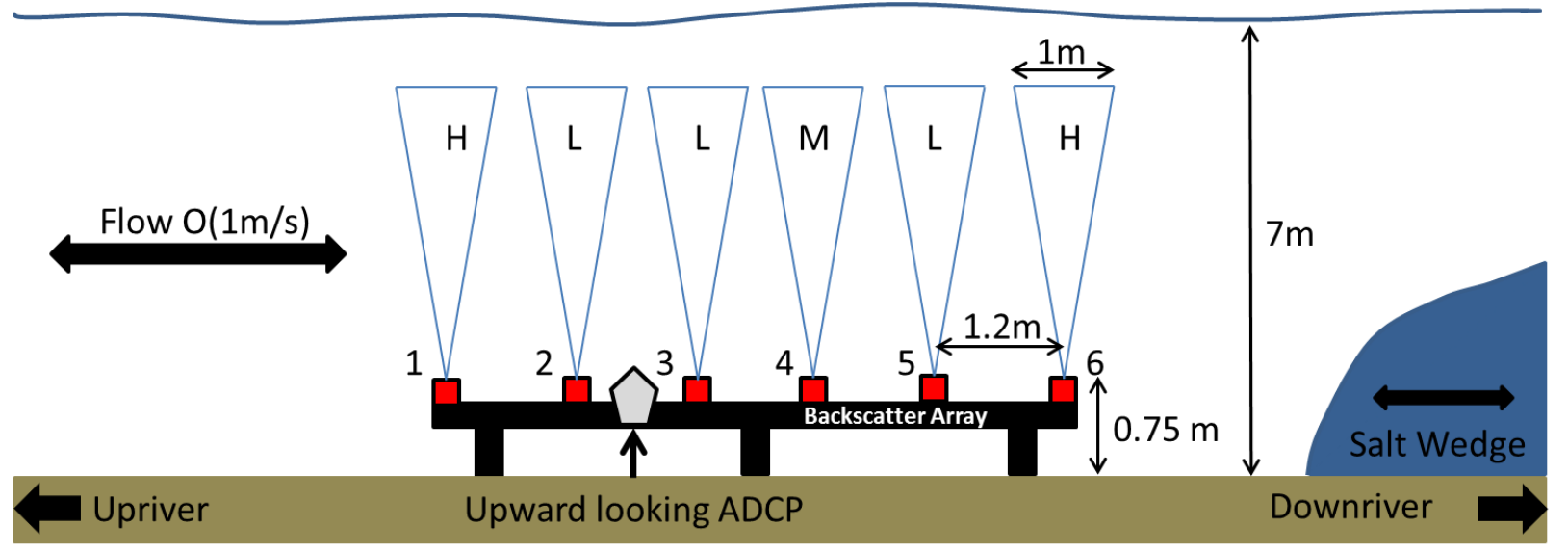

Figure 2. Schematic of the bottom-mounted, six-transducer, high-frequency, broadband acoustic backscatter array. The transducers are labeled 1 through 6 , with transducer 1 being most up-river. The labels $\mathrm{H}, \mathrm{L}$ and $\mathrm{M}$ indicate the frequency band of each transducer (table1). The acoustic sampling volumes, determined by the beam width and frequency of each transducer, do not overlap at any range. The salt wedge advects over the array from transducer 6 to 1 during flood tides and is mixed away over the array during the ebb tides.

The transducers on the array were evenly space 1.2 meters apart along a six-meter long rigid steel frame (Figure 2). The steel frame was aligned with the flow and had weighted feet which secured it to the bed of the river. The acoustic system on the array was cabled to the R/V Connecticut providing power as well as real-time data acquisition.

The broadband transducers, signals and processing techniques result in high vertical range resolution and enhanced target discrimination capabilities compared to narrowband transducers, signals and processing techniques. Narrowband systems have a range resolution dependent on the length of the transmitted signal and sound speed of the acoustic medium. In contrast, by using pulse compression signal processing techniques [Chu and Stanton, 1998], the range resolution of broadband systems is independent of the transmitted signal length and is determined by the bandwidth of the transmitted signal. Three different center frequency transducers were attached to the array (Table 1). In combination, the transducers spanned the frequency range of $160-590 \mathrm{kHz}$, with some gaps. For the purposes of this paper, the 160-270 $\mathrm{kHz}$ transducers are designated as $\mathrm{L}$, the $220-300 \mathrm{kHz}$ transducers are designated as $\mathrm{M}$, and the 430-590 kHz transducers are designated as $\mathrm{H}$ (Table 1, Figure 2). In terms of enhanced target 
discrimination, while narrow bandwidth systems result in volume backscatter strength at one frequency, broadband systems provide volume backscatter strength information over a continuous range of frequencies from each transducer. Combing the backscatter strength information from each transducer associated with the system results in a nearly continuous coverage of backscatter strength over the total bandwidth $(160-590 \mathrm{kHz})$ of the system.

Table 1. Specifications for the 6 transducer mounted on the array.

\begin{tabular}{|c|c|c|c|c|c|c|}
\hline $\begin{array}{c}\text { Transducer } \\
\text { Number }\end{array}$ & 1 & 2 & 3 & 4 & 5 & 6 \\
\hline $\begin{array}{c}\text { Frequency } \\
\text { Band }\end{array}$ & $\mathrm{H}$ & $\mathrm{L}$ & $\mathrm{L}$ & $\mathbf{M}$ & $\mathrm{L}$ & $\mathrm{H}$ \\
\hline $\begin{array}{c}\text { Frequency } \\
\text { Range } \\
(\mathrm{kHz})\end{array}$ & $\begin{array}{c}450 \\
- \\
590 \\
\end{array}$ & $\begin{array}{c}160 \\
- \\
270 \\
\end{array}$ & $\begin{array}{c}160 \\
- \\
270 \\
\end{array}$ & $\begin{array}{c}220 \\
- \\
330 \\
\end{array}$ & $\begin{array}{c}160 \\
- \\
270 \\
\end{array}$ & $\begin{array}{c}450 \\
- \\
590 \\
\end{array}$ \\
\hline $\begin{array}{l}\text { Full Beam } \\
\text { Width } \\
\text { (degrees) }\end{array}$ & 7.2 & 9.3 & 9.3 & 10 & 9.3 & 7.2 \\
\hline
\end{tabular}

An acoustic image (Figure 3) consists of the envelope of the pulse compression signal processing for successive pings from a single transducer plotted as a function of range. A ping consists of a transducer transmitting a $500 \mu$ s chirp and recording the received backscatter signal for approximately $15 \mathrm{~ms}$ after transmitting. Each ping provides full water column coverage of the scattering processes at $1.5-3.0 \mathrm{~cm}$ range resolution, depending on the bandwidth of the transmitted signal. During the experiment each transducer pinged at $7 \mathrm{~Hz}$ with a $100 \mathrm{~ms}$ between transducers 4, 5 and 6 and 1,2 and 3 transmitting. The volume of water sampled by each transducer depends on the transducer beam width, frequency and range from the transducer. For the ranges involved with this experiment the acoustic sampling volume did not overlap at any range (Figure 2). 


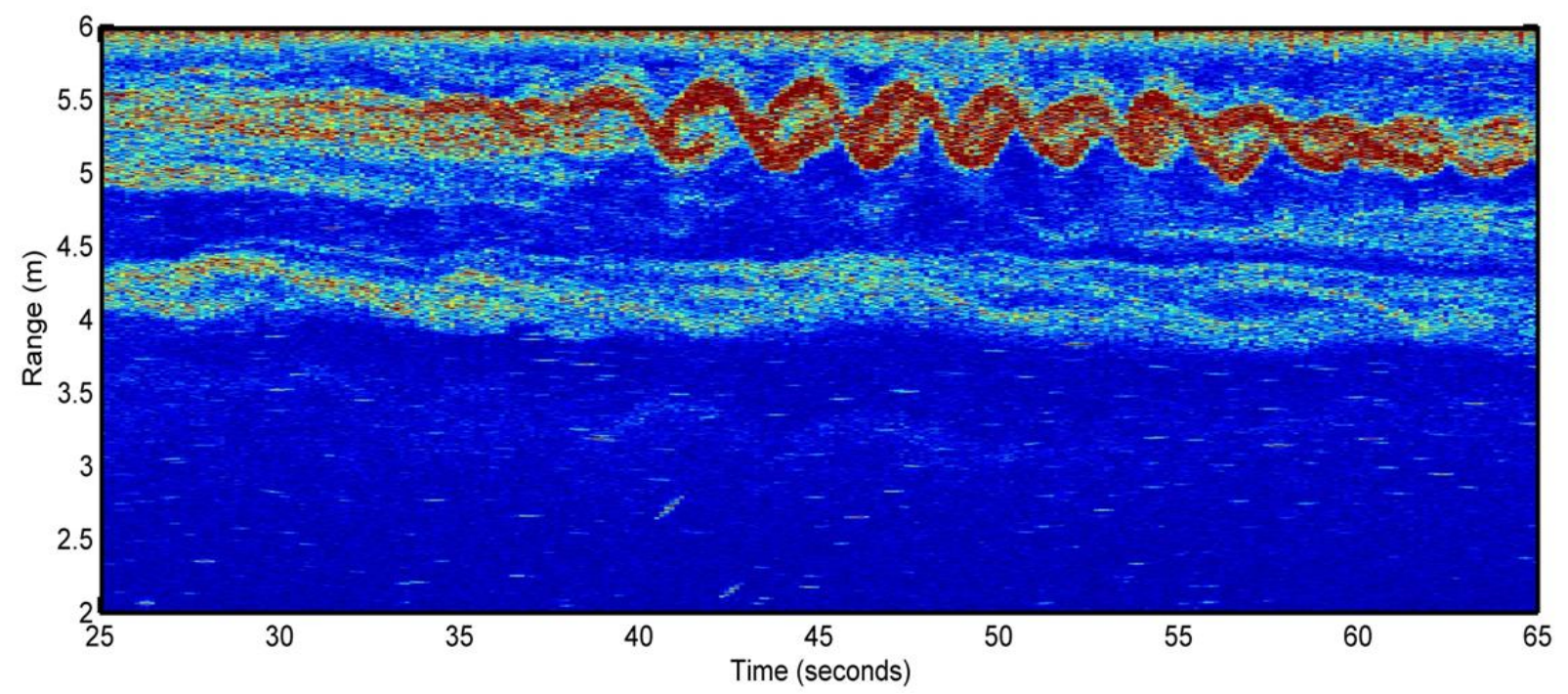

Figure 3. Acoustic image from transducer 6 showing a packet of stratified shear instabilities that occurred during the morning ebb tide on December $7^{\text {th }}$ (Observation A). The range is distance from the backscatter array. The backscatter is high in regions of strong density gradients [Lavery et al., 2013].

Current profiles were recorded every $30 \mathrm{~s}$ with $0.25 \mathrm{~m}$ vertical resolution using the ADCP mounted on the array (Figure 2). Conductivity and temperature profiles were obtained with the CTD deployed by hand from the R/V Connecticut which was anchored $\sim 200 \mathrm{~m}$ to the west of the array. CTD profiles were performed repeatedly for five minutes three times an hour throughout the entire experiment.

\section{Quantification of shear instabilities: kinematics}

The acoustic images (Figure 3) generated at each transducer on the array are used to track the location as well as estimate the amplitude and wavelength of individual shear instability as they advect over the array. Shear instabilities appear as regions of strong backscatter in the acoustic images (Figure 3), displaying a structure similar to what is seen in laboratory and numerical experiments. The amplitude and period of the waves are directly estimated from the acoustic images. Wavelength is determined using measurements of velocity from the ADCP at the location of the shear instabilities in conjunction with the period of the waves estimated from the image in Figure 3. Details on how these different parameters are estimated are forthcoming. 
A custom edge detection algorithm applied to the acoustic images is used to estimate the wavelength and amplitude of the waves as well as track them as they advect over the array.

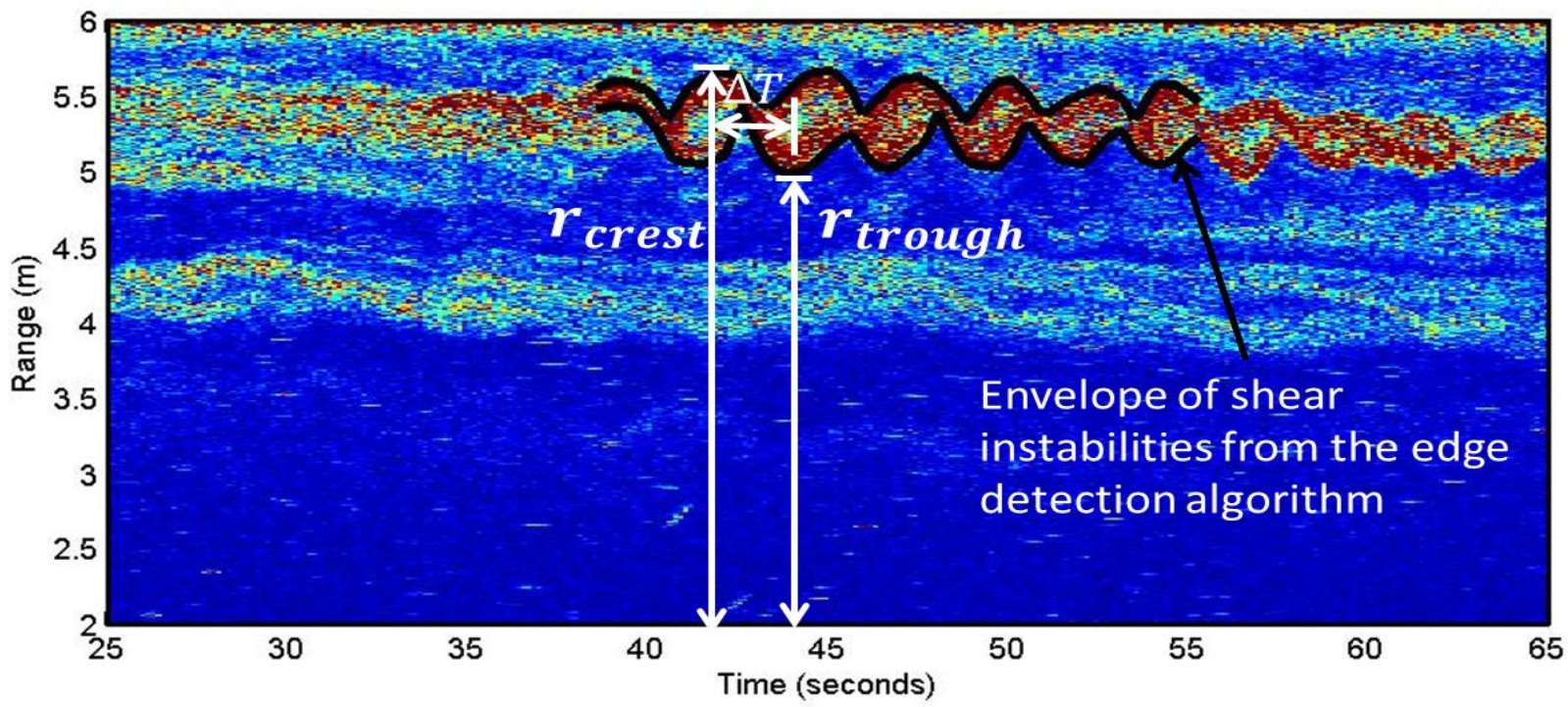

Figure 4. Acoustic image from transducer 6 during the morning ebb tide on December $7^{\text {th }}$ (Observation A) with the output of the edge detection algorithm marking the packet of shear instabilities. The amplitude and wavelength is estimated using the edge detection output at each transducer on the array, this is an example of analysis of an image from one transducer.

The amplitude of the waves is found by taking the difference in the range of the crest and trough of the shear instability (Figure 4). The amplitude of a wave as it passes each transducer is defined as $h=r_{\text {crest }}-r_{\text {trough }}$ (Figure 4). The wavelength is estimated by calculating the time $(\Delta T)$ between the arrival of the crest and trough and multiplying $\Delta T$ by the velocity measured by the $\mathrm{ADCP}$ at the mean range of the shear instability $\left(V_{A D C P}\right)$ i.e., $\lambda=\Delta T V_{A D C P}$. The mean range is defined as $\left(r_{\text {crest }}+r_{\text {trough }}\right) / 2$.

The spatial separation of the transducers on the array makes it possible to quantify the spatial and temporal evolution of individual shear instabilities. The evolution is quantified by measuring $h$ and $\lambda$ for an individual wave at each transducer along the array. This is achieved by tracking the wave from one transducer to the next along the array. The tracking is achieved by cross-correlating the edge detection outputs (Figure 4) from an upstream transducer with a 
downstream transducer and using the lag time at the peak in the correlation to find the location of the shear instability in the image from the downstream transducer. To ensure each wave is accurately tracked the velocity of the shear instability is compared to the velocity measured by the ADCP at the location of the shear instability. Both velocity estimates should be nearly equal. Once the wave is tracked from the upstream transducer to the downstream transducer, its amplitude and wavelength can be estimated again at the downstream transducer. This process of quantifying the wave at one transducer and tracking it to the next is repeated until the wave reaches the end of the array.

As a result of the array's finite length, the evolution of waves can only be well resolved for waves with an evolution time on the order of their advection time past the array. Shear instabilities that evolve slowly compared to their time to advect over the array will appear as if they are not evolving. In contrast, if a wave evolves quickly compared to the advection time past the array, its evolution may be under resolved by the array. Wave evolution is best resolved for waves with wavelengths of 1-3 m using this array.

\section{Quantification of mixing: acoustic inversions}

In addition to providing high resolution images, the broadband electronic system and transducers on the array are calibrated which enables inversions for turbulent kinetic energy dissipation rate $(\varepsilon)$ to be made. The transducers and broadband system on the array were calibrated using standard target techniques following Lavery et al. [2010, 2013]. Enhanced spectral discrimination between the scatterers is achieved by combining high-frequency scattering models with the use of calibrated broadband transducers and broadband system. 
Following Lavery et al. (2013) quantitative inversions for turbulent dissipation and salinity variance can be made once the scattering mechanism has been identified.

The volume backscattering coefficient, with units of inverse length, as function of frequency measured from a single transducer is given by [Lavery et al., 2010]

$$
\sigma_{V}(\omega)=\frac{\left\langle\left|V_{v o l}^{F F T}(\omega)\right|^{2}\right\rangle}{\operatorname{Cal}(w)\left|L_{v o l}(\omega)\right|^{2}}
$$

where $V_{v o l}^{F F T}(\omega)$ is the Fourier transform of a specific range of data within a ping, $\operatorname{Cal}(w)$ is the frequency dependent calibration curve for the given transducer and channel on the electronics system. $\operatorname{Cal}(w)$ corrects for spherical spreading, attenuation associated with the calibration measurements and the response of the transducer and backscatter system and $\left|L_{v o l}(\omega)\right|^{2}$ corrects for spherical spreading and attenuation. $\sigma_{V(\omega)}$ is estimated by selecting a patch of data from the acoustic images and applying Equation 1 to each ping within the patch. A patch consists of tens of consecutive pings with overlapping or identical range gates. An example of a patch of data is the data associated with the waves in Figure 4 within the output of the edge detection algorithm. Estimates of $\sigma_{V}(\omega)$ from multiple transducers (e.g. a $\mathrm{L}$ and $\mathrm{M}$ transducer) are used to create a spectrum that spans a larger fraction of the frequency spectrum which can increase confidence in scattering mechanism discrimination and quantification.

The volume backscatter cross-section as a function of frequency from multiple transducers, referred to as a backscatter spectrum, is estimated from transducers 3 (L) and 4 (M) on the array. In previous studies (Lavery et al. 2013) transducers that ensonify a single volume of fluid are used to estimate $\sigma_{V}(\omega)$ over a broad range of frequencies to enable the resulting backscatter spectrum to be interpreted as a discretely sampled continuous backscatter spectrum [Lavery et al., 2010; Lavery et al., 2013]. However, the transducers on the array are separated by 
$1.2 \mathrm{~m}$ and consequently do not sample the same volume of fluid at the same time. It is known that the flow and therefore the scattering processes are inhomogeneous over $1 \mathrm{~m}$ length scales in the Connecticut River (Figure 4), suggesting a backscatter spectrum formed using pings coincident in time from transducers 3 and 4 cannot be interpreted as a discreetly sampled continuous spectrum. To get around this problem, the backscatter spectrums are formed from volume backscatter associated with a particular coherent structure in the images, such as shear instabilities, which can be tracked from transducer to transducer. The patch of data associated with a particular coherent structure at the upstream transducer is used to estimate $\sigma_{V}(\omega)$ at the upstream transducer. At the downstream transducer, the particular coherent structure is identified and the patch of data associated with the structure is used to estimate $\sigma_{V}(\omega)$ at the downstream transducer. Combining the two estimates of $\sigma_{V}(\omega)$, which cover different frequency bands, results in a backscatter spectrum. Since the data is selected from an advecting coherent structure it is assumed that the same scattering mechanism and nearly the same volume of fluid is sampled at each transducer. Because there is a time delay between ensonfications it must also be assumed that the amplitude of $\sigma_{V}(\omega)$ does not change significantly during the time it takes for the coherent structure to travel between transducers. It is known there are some changes in amplitude but it is assumed they have minimal effects on classifying and quantifying volume backscattering mechanisms.

When the measured backscatter spectrum is consistent with stratified homogeneous and isotropic turbulence, salinity variance and turbulent kinetic energy dissipation rate can be inferred by assuming a known flux Richardson number $R_{f}$ following Lavery et al. [2013]. The assumption of constant $R_{f}$ is still debated, although many investigators of stratified turbulence apply the value obtained by Osborn [1980] of 0.15, which is also used in this study. For the 
transducer frequency bands employed on the array it is expected backscatter will be from stratified turbulence in the viscous convective sub-range. The volume backscattering crosssection as a function of frequency for homogeneous isotropic stratified turbulence in the viscousconvective sub-range is given by Eq. 6 in Lavery et al. [2013]

$$
\sigma_{V}^{v c}=2^{-3} \Psi^{a c} q \chi_{s}\left(\frac{\varepsilon}{v}\right)^{-1 / 2} k
$$

where $\sigma_{V}^{v c}$ is the backscattering cross section per unit volume, with units of inverse length, $\Psi^{a c}$ $\left(1 / p s u^{2}\right)$ parameterizes changes in salinity and temperature due to changes in sound speed and density, as well as changes due to the temperature and salinity co-spectrum [Lavery et al., 2013], $q$ is an empirical constant $(q=0.2)$ [Lavery et al., 2013], $\chi_{s}\left(\mathrm{psu}^{2} / \mathrm{s}\right)$ is the salinity variance and $k(1 / \mathrm{m})$ is the acoustic wave number. Equation 2 can be re-written for $\varepsilon$ assuming a known flux Richardson number, $R_{f}=0.15$, as

$$
\varepsilon=\left(\frac{\sigma_{V}^{v c}}{2^{-3} \Psi^{a c} q k}\right)^{2}\left(\frac{g \beta}{2 R_{f}}\right)^{2}\left(\frac{\partial \bar{S}}{\partial z}\right)^{-2} v^{-1}
$$

where $\beta=(1 / \rho)(\partial \rho / \partial \bar{S}), g$ is the gravitational constant and $\partial \bar{S} / \partial z$ is the mean salinity gradient. Equation 3 is used to estimate the change in $\varepsilon$ as the shear instabilities evolve.

\section{Results}

This study quantifies the spatial and temporal evolution of 26 shear instabilities. The instabilities occurred during the first 1-2 hours of an ebb tide and have wavelengths and amplitudes of 1-3 m and 0.4-0.7 m respectively (Table 2). A typical $R e$ is approximately 50,000. Hundreds of shear instabilities with amplitudes and wavelengths as large as 2.5 and $20 \mathrm{~m}$, respectively, are observed each tidal cycle. The 26 waves, which occurred in five packets (labeled Observations A-E), presented here displayed clear enough evolution as they advected over the array to provide reliable estimates of growth rate. As explained in the Methods section, 
one reason so few of the waves exhibited evolution as they passed over the array is the array's finite length.

Observation A consists of a packet of seven consecutive shear instabilities (Figure 5), which occurred on 7 December 2012, approximately two hours after the ebb tide began. The acoustic images from successive transducers on the array capture the wavelength and amplitude of the waves as they advect over the array. The amplitude of the waves increases from transducer 1(the most upstream) to transducer 6 (the most downstream) (Figure 5). Each wave takes approximately $6 \mathrm{~s}$ or 3 shear time scales $(\tau=\Delta z / \Delta u[s])$ to advect over the array and reach a final amplitude of $0.6 \mathrm{~m}$ (Figure 5). The wavelength of the waves is $2.2 \mathrm{~m}$ with a standard deviation of $0.2 \mathrm{~m}$ (Table 2).

Particular attention should be paid to waves 1 and 2 because of the two distinct phases of evolution they exhibit. Wave 1 demonstrates the growth regime and wave 2 is an example of a wave that stops growing without collapsing. Wave 1 is first observed as what appears to be a scattering layer (Transducer 1 in Figure 5) and wave 2 is first observed as a finite amplitude wave. Wave 1 grows as it advects over the array to a final observed amplitude of $0.5 \mathrm{~m}$ (Figure 6). During its growth, wave 1 demonstrates an increase in amplitude but no periodic doubling or other obvious wave coupling behavior. In contrast to wave 1, the amplitude of wave 2 remains nearly constant as it advects over the array. This will be referred to as the arrested growth regime. Further, wave 2 shows no signs of overturning or breaking (Figure 5).

The evolution of the remaining waves from Observation A can be described as a combination of the evolutions of waves 1 and 2. The zero and/or slowing growth rate of waves three, four and five as they reach the end of the array suggests that they are approaching the same regime as wave 2 (Figure 6). Waves six and seven do not appear to reach the arrested growth 
regime, but this may be because they are growing slightly slower or growing to a larger

amplitude than the other waves in Observation A. Slightly slower growth rate or larger final amplitude would result in less of their evolution being captured by the array. The timescale of growth for all the waves in Observation $\mathrm{A}$ is similar to the growth time scale of wave number 1 (Figure 6).

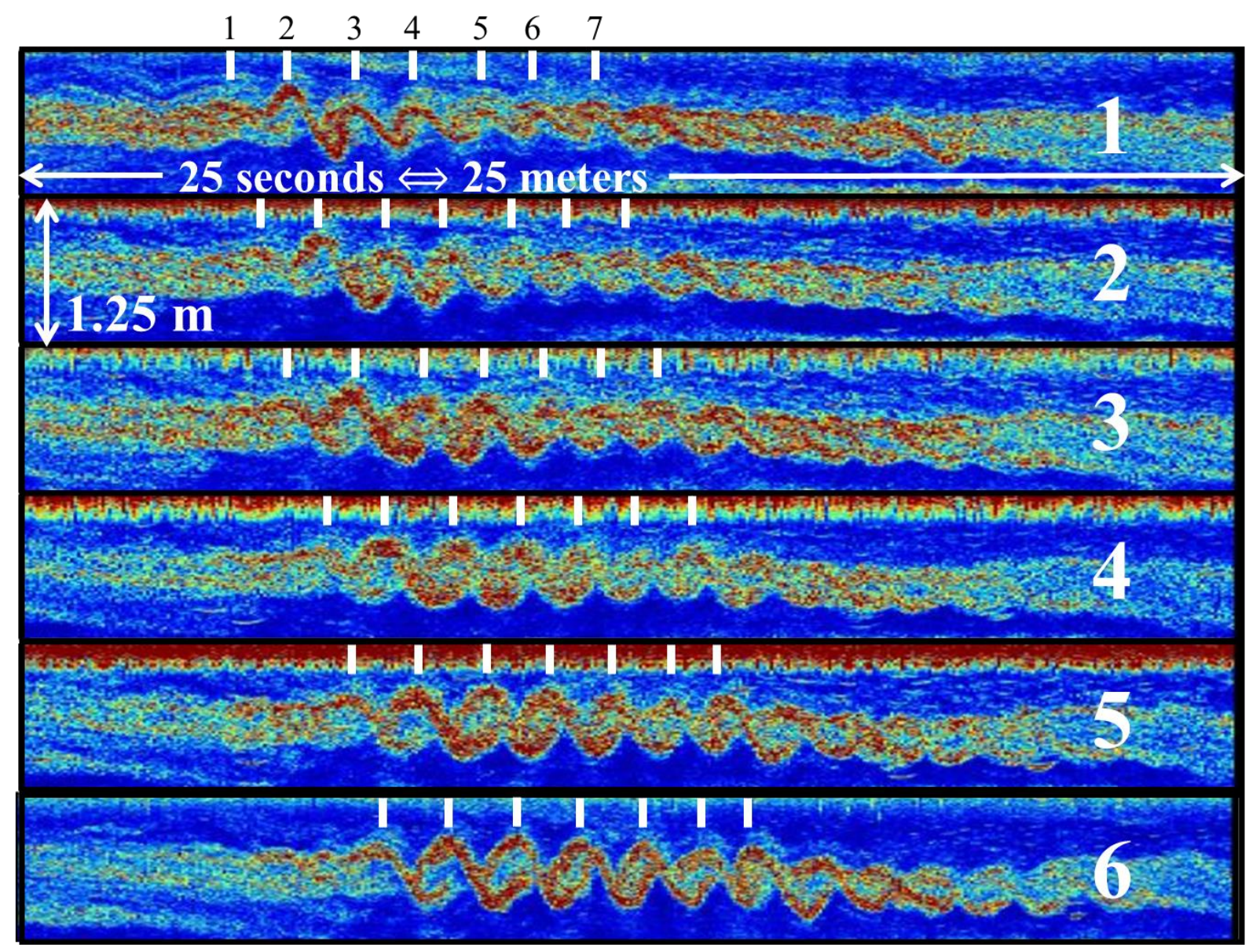

Figure 5. Acoustic images of shear instabilities from all six transducers on the array. The vertical range for each record is $1.25 \mathrm{~m}$ and the horizontal axis is $25 \mathrm{~s}$ in time which corresponds to approximately $25 \mathrm{~m}$ in space using the velocity from the ADCP. The individual waves are labeled 1-7 at the top of the figure. Most of the waves grow as they pass from transducer 1 to 6 (labeled on right hand side). Flow is from right to left and the faster fresher water overlies slower, saltier, denser water at the bottom of the images.

The mean slopes $\left(h_{\max } / \lambda\right)$, where $h_{\max }$ is the maximum amplitude the wave reaches, of the waves in Observation A (Table 2) are in the range of those observed in laboratory, numerical and previous field Observations [Thorpe, 1973; Smyth, 2003; Wroblewski et al., 2007]. It is also 
found that the advection velocity of the waves $\left(V_{\text {array }}^{\text {wave }}\right)$ and velocity measured by the ADCP are in close agreement as expected (Table 2).

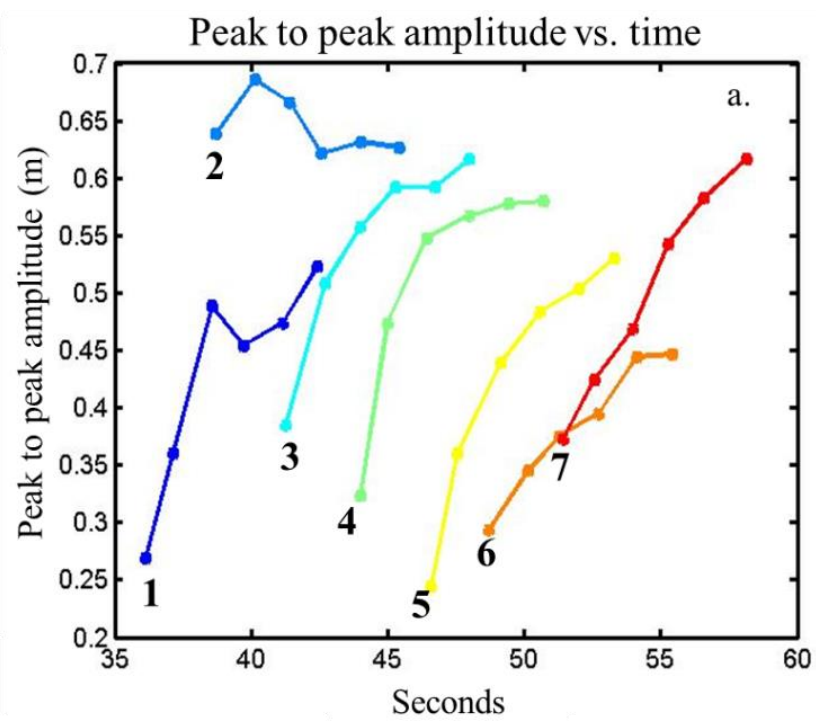

Figure 6. Peak-to-peak amplitude of the stratified shear instabilities shown in Figure 4.

Table 2. Estimates of $R i_{\text {local }}, V_{A D C P}, V_{\text {array }}^{\text {wave }}, h_{\max }$ and $\lambda$ inferred from the combination of data from the array, $\mathrm{ADCP}$ and CTD. The $R i$ estimates are made from the CTD and ADCP Observations. $R i_{\text {local }}$ is $R i$ estimated at the mean range of shear instabilities. $V_{\text {array }}^{\text {wave }}, h_{\max }$ and $\lambda$ are estimated from the acoustic records. Note the close agreement of $V_{A D C P}^{\text {wave }}$ and $V_{\text {array }}^{\text {wave }}$.

\begin{tabular}{|c|c|c|c|c|c|}
\hline Observation & $\mathrm{A}$ & $\mathrm{B}$ & $\mathrm{C}$ & $\mathrm{D}$ & $\mathrm{E}$ \\
\hline time & $2012 / 12 / 07$ & $2012 / 12 / 05$ & $2012 / 12 / 06$ & $2012 / 12 / 05$ & $2012 / 12 / 05$ \\
& $07: 19 \mathrm{EST}$ & $05: 41 \mathrm{EST}$ & $19: 04 \mathrm{EST}$ & $05: 39 \mathrm{EST}$ & $17: 35 \mathrm{EST}$ \\
\hline$R i_{\text {local }}$ & 0.26 & 0.03 & 0.27 & 0.44 & 0.22 \\
\hline$V_{\text {ADCP }}(\mathrm{m} / \mathrm{s})$ & 0.94 & 0.57 & 0.63 & 0.99 & 0.93 \\
\hline$V_{\text {array }}^{\text {wava }}(\mathrm{m} / \mathrm{s})$ & 0.93 & 0.52 & 0.86 & 0.97 & 1.0 \\
\hline$h_{\max }($ mean $)(\mathrm{m})$ & 0.56 & 0.75 & 0.57 & 0.42 & 0.40 \\
\hline$h_{\max }(\mathrm{std})(\mathrm{m})$ & 0.08 & 0.12 & 0.08 & 0.11 & 0.14 \\
\hline$\lambda(\mathrm{m})$ & 2.2 & 4.3 & 1.7 & 2.4 & 1.5 \\
\hline$\lambda(\mathrm{std})(\mathrm{m})$ & 0.2 & 0.5 & 0.2 & 0.3 & 0 \\
\hline$h_{\max }($ mean $) / \lambda$ & 0.28 & 0.18 & 0.33 & 0.18 & 0.27 \\
\hline
\end{tabular}

A density profile was measured $120 \mathrm{~s}$ before the shear instability arrived and a velocity profile was measured $13 \mathrm{~s}$ before the shear instabilities reached the array. The profiles show the water column is strongly sheared and strongly stratified (Figure 7). Estimates of $R i$ from the profiles show that a $R i$ value close to the stability threshold of 0.25 occurs in the upper water 
column at the same range as the shear instability from Observation A (highlighted by the gray box in Figure 7, Figure 4). There are other locations in the $R i$ profile with local minima as well as $R i$ values less than 0.25 . At $4 \mathrm{~m}$ range, $R i$ is less than 0.25 , and shear instability are observed in the acoustic images at this range (Figure 4). However, these waves have a lower backscatter signal making it difficult to track them and therefore are not included in this analysis. At $1 \mathrm{~m}$ range $R i$ is also less than 0.25 , but no shear instability is detected at this range, possibly because stratification is too weak to generate detectable acoustic backscatter (Figure 7).

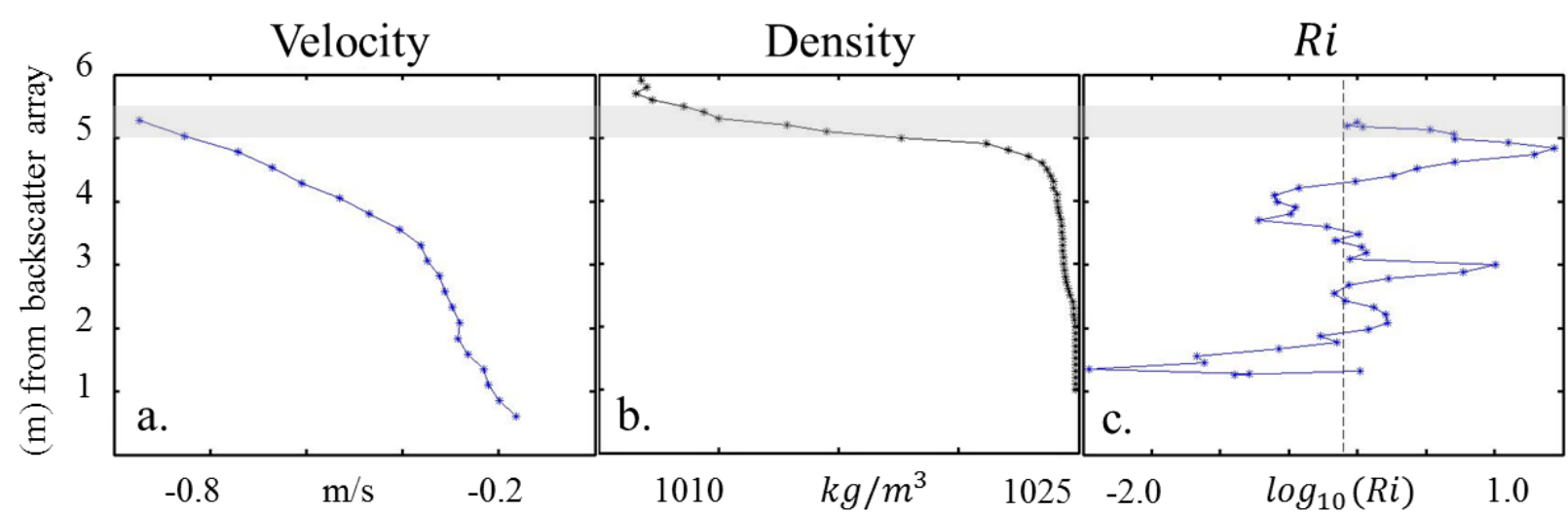

Figure 7. Velocity, $R i$, and density profiles for the stratified shear instability from Observation A (Figure 2). a.) velocity profile obtained from the ADCP on the array, b.) Density profile, inferred from the CTD data. c.) Estimates of $R i$ using the profiles from a.) and b.).

Estimates of $R i$ from the ADCP and CTD do not provide an accurate quantification of $R i_{o}$, because the estimates are made as the shear instabilities are evolving and the ADCP and CTD are not co-located in time or space. Offsets in time and space of the density and velocity profiles almost certainly introduce errors into the estimate of $R i$. Additionally, the range resolution of the ADCP is nearly identical to the shear layer thickness expected for the wavelength of the waves observed which means the velocity profile are too course to estimate $R i_{o}$ for the shear instability.

The majority of the waves in Observations B through E exhibit a combination of the evolution characteristics revealed by waves 1 and 2 from Observation A (Figure 8, 9). As seen in 
Observation A the waves also appear in packets (Figure 8). In addition, Observations B through E have similar $R i$ (obtained from CTD and ADCP data), wavelengths, final amplitude and therefore slope (Table 2). Observations B through E evolve on shear time scales nearly identical to those in Observation A (Table 3) as they initially grow rapidly and slow or stop growing by the time the end of the array is reached (Figure 9, Table 3). Further, the waves do not exhibit collapse or roll-up once their growth has stopped (Figure 9). As the waves grow, there is no sign of periodic doubling either. The two-dimensional structure of waves from Observation B through E exhibit similar characteristics to Observation A (Figure 8). Most notably, the strongest backscatter is located in the braid regions of the waves for Observations B through E. Additional figures in Appendix 1 show more detailed results for Observations B through E.
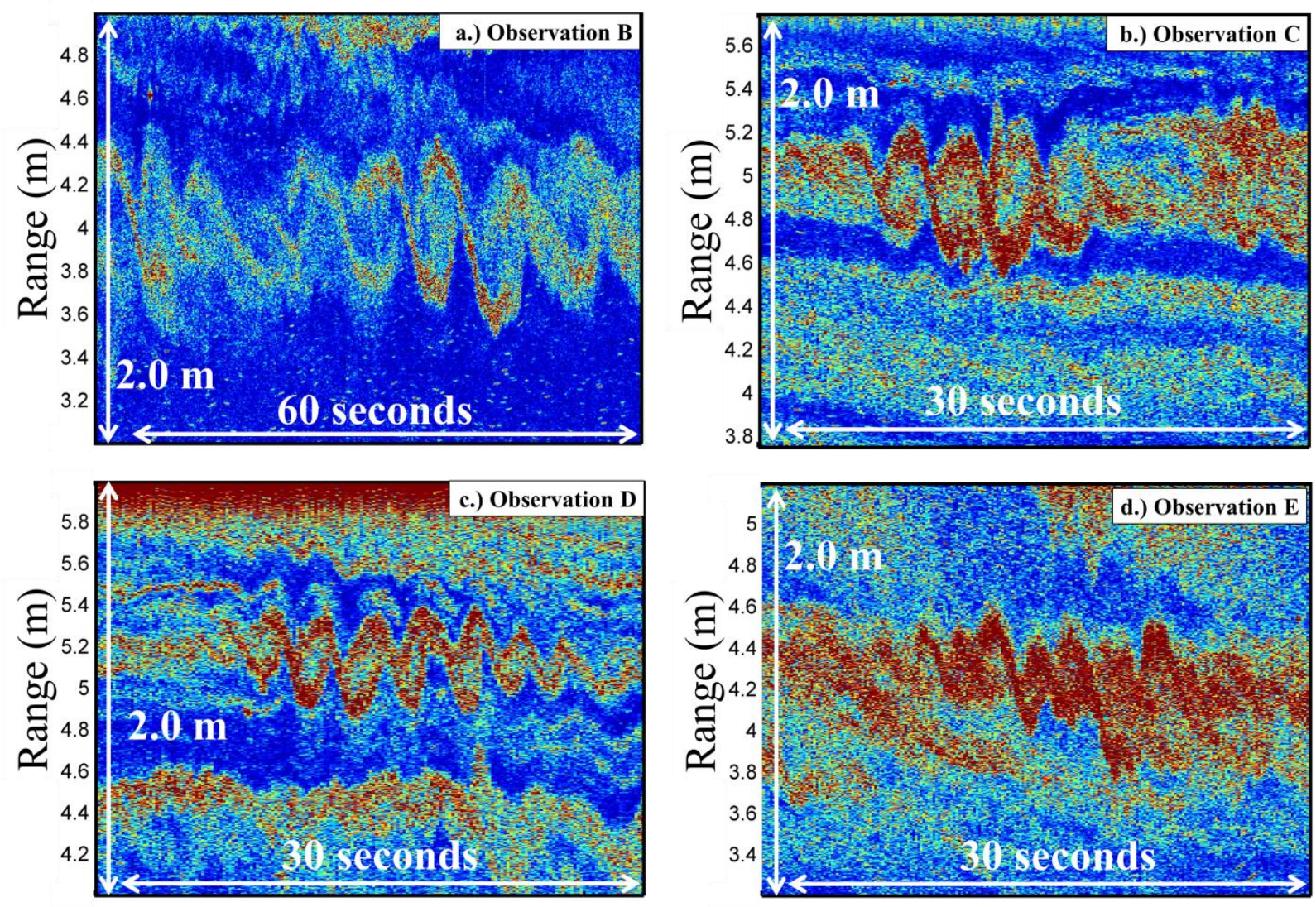

Figure 8. Acoustic images showing the growing shear instabilities from Observations B through E. a-d) Shear instability analyzed from three different days during similar phases of the ebb tide. $R i, h_{\max }$ and $\lambda$ were calculated for each of these examples. It can also be seen that the strongest backscatter occurs in the braids of these shear instabilities. All images are from transducer 6 . 
Table 3. Shear time scales for each Observation. The * indicates $\Delta u$ was not adequately resolved by the ADCP and was deduced from averaging two estimates of $\Delta u$. One estimate is from a linear interpolation of the velocity profile and the other deduced from the $R i_{o}$ estimates made in the discussion section.

\begin{tabular}{|c|c|c|c|c|c|}
\hline Observation & A & B & C & D & E \\
\hline$\tau(\mathrm{s})$ & $2.3^{*}$ & 3.8 & 2.4 & $2.2^{*}$ & 1.7 \\
\hline
\end{tabular}
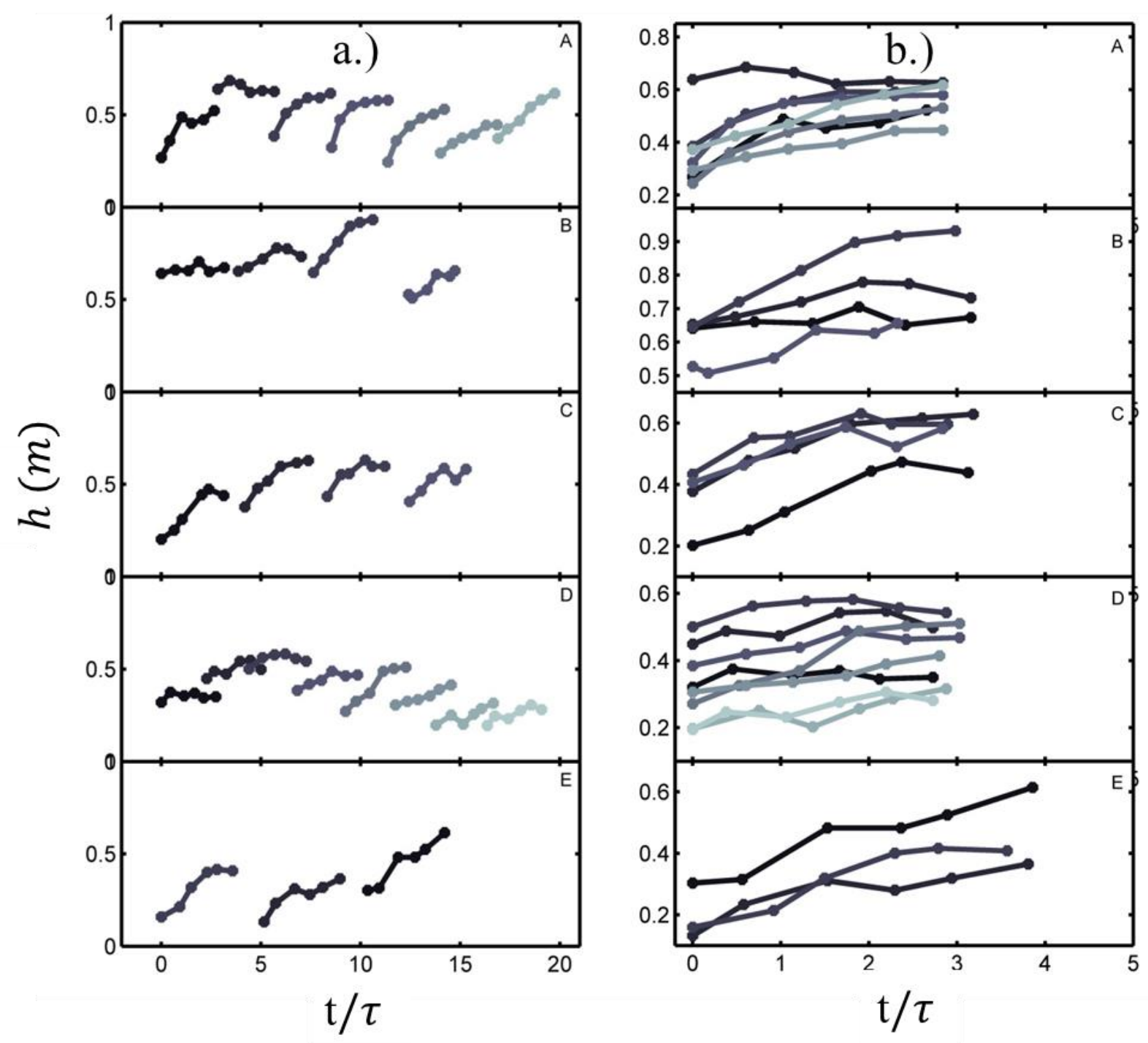

Figure 9. a.) Amplitude $h(m)$ vs. shear time scales $(t \backslash \tau)$ for all the stratified shear instabilities from Observations A through E during the December 2012 experiment in the Connecticut River. b.) same data as in a.) but with all the curves starting at the same time and varying y-axis ranges for each Observation. Nearly all of the stratified shear instabilities are growing and most of them exhibit the arrested growth regime. On average the shear instabilities grow by a factor of 1.7 times their initial measured amplitude with a standard deviation of 0.4

The arrested growth phenomenon of shear instabilities is not clearly observed as often as their growth phase although many of the Observations indicate a stage in which the growth rate 
decreases. Of the remaining Observations, only Observation B contains a wave which maintains a constant or decreasing amplitude as it advects over the array like wave 2 from Observation A (Figure 9). It could be the result of two factors: the array resolves only a part of the waves' evolution, and the waves from Observations A and B could be reaching the array at just the right time for the array to detect the arrested growth phenomena. Despite only two cases of waves exhibiting clear arrested growth over the array, many of the observed waves have zero or negative growth for some fraction of their observed evolution (Figure 9). Additionally, many of the waves that continued to grow as they reach the end of the array were growing slower than when they first entered the view of the array. With a longer array, the arrested growth regime would perhaps be more frequently observed.

Thus far the array’s imaging capabilities have been used to extract information about the evolution of stratified shear instabilities. Now the volume backscatter strength will be analyzed to classify the scattering in the water column and quantify the intensity of the salinity microstructure.

The backscatter spectrum for Observation A is consistent with homogeneous isotropic stratified turbulence (Figure 10). The edge detection algorithm is used to select the data for spectral analysis to ensure that only backscatter from the waves is considered (Figure 10a). The slope of the resulting backscatter spectrum is 1.6 (Figure 10b), which is significantly different from the slope of other potential scattering mechanisms. The backscatter is likely from homogeneous isotropic stratified turbulence in the viscous convective sub range as given in equation 1 [Lavery et al., 2014]. Based on this information and the strong shear and stratification in the water column (Figure 5), it is assumed that the backscatter from all the waves in Observation A comes from homogeneous isotropic stratified turbulence. Using this assumption, 
the backscatter spectrum can be compared between transducers 1 and 6 . By further assuming a value of the mixing efficiency and estimating the average salinity gradient within the instability, $\varepsilon$ can be estimated using equation 2 [Lavery et al., 2013].

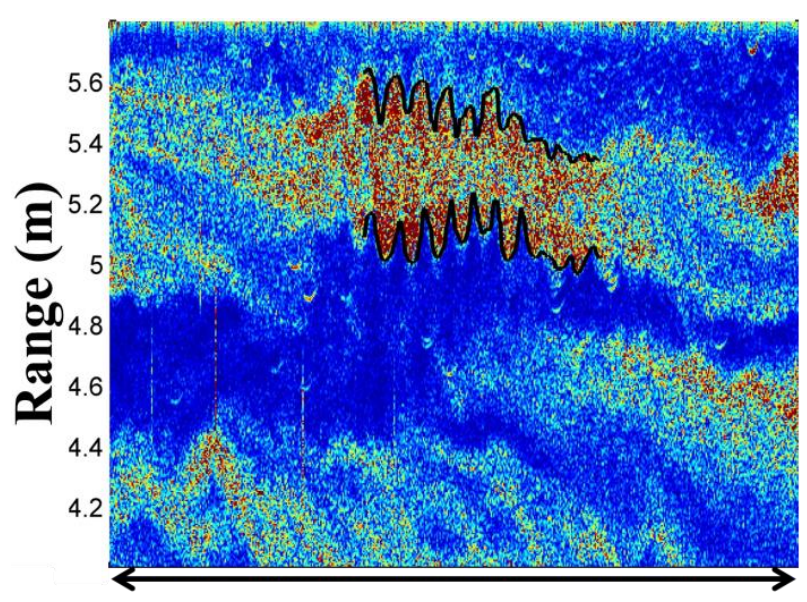

70 seconds

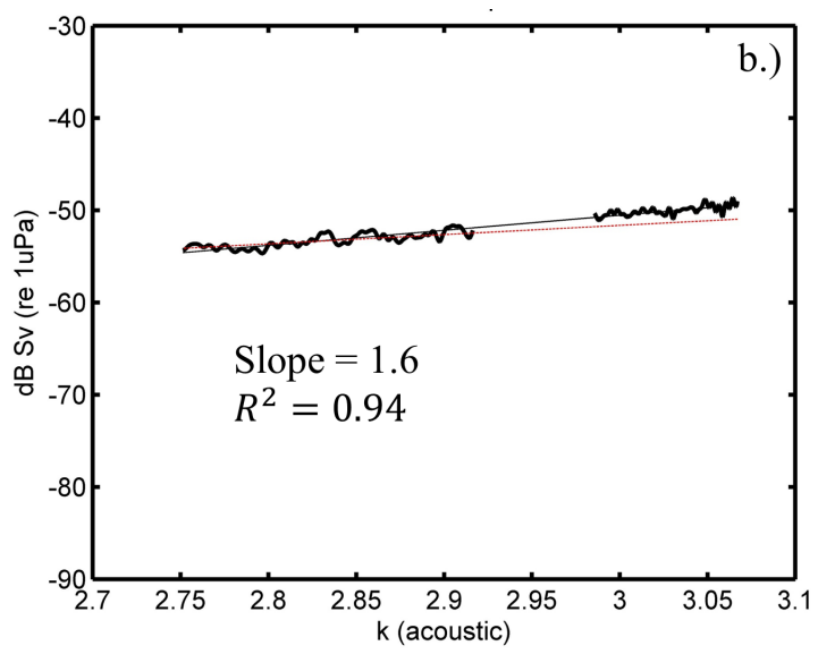

Figure 10. Spectral classification of the backscatter associated to the shear instabilities. a.) Acoustic image from transducer 4 with the black outline illustrating the section of data analyzed. b.) Result of the spectral analysis, the spectrum is consistent with backscatter from stratified turbulence, the dashed line is the theoretical slope for volume backscatter from stratified turbulence in the viscous convective sub-range, $S_{V}(\omega)=10 \log _{10}\left(s_{V}(\omega)\right)$. Transducers 3 and 4 were used to create this spectrum.

Comparing the backscatter spectrum at transducer 1 (upstream) with transducer 6 (downstream) shows the amplitude of the backscatter spectrum is larger at transducer 6 (Table 4). Figures 11a and 11b show the region of data selected for analysis from transducers 1 and 6 . The same waves are selected in each image. A factor of 2.3 change in the volume backscattering strength between transducer 1 and 6 is observed. Assuming all other terms in equation 3, like the mean salinity gradient, are constant, $\varepsilon$ increases from $4.8 \times 10^{-5} \mathrm{~m}^{2} / \mathrm{s}^{3}$ to $2.5 \times 10^{-4} \mathrm{~m}^{2} / \mathrm{s}^{3}$, which is a factor of 5 change in $\varepsilon$ (Table 4).

This analysis demonstrates that the turbulence in the braids increases as the growth rate of the waves decreases. The growth rate of waves from Observation A decreases as they advect past the array (Figure 7). Simultaneously $\varepsilon$ is increasing as deduced from the acoustic 
backscatter. These data did not show a correlation between growth rate and $\varepsilon$, however, such a correlation may be observed with a wider range of the two variables.

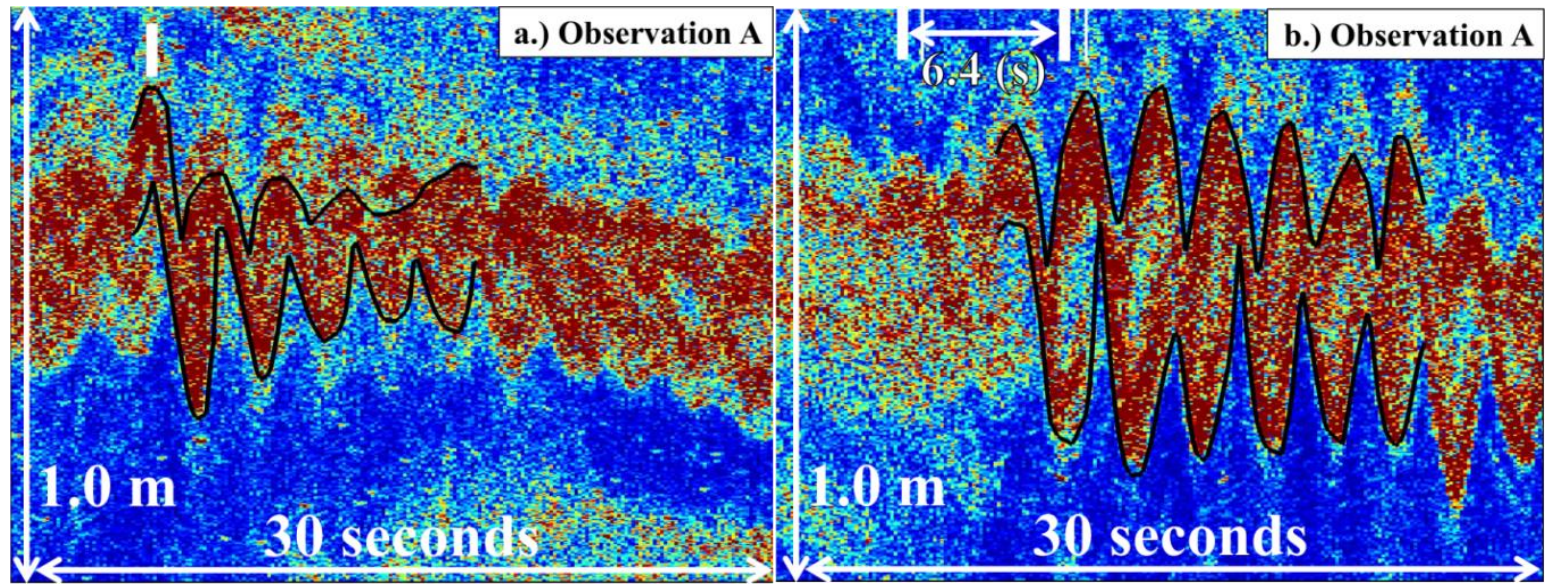

Figure 11. Data selected from transducer 1 and transducer 6 to make estimates of the change in backscatter strength. a) The section of data analyzed from transducer 1 outlined by the black line. b) The section of data analyzed from transducer 6 outlined by the black line

Table 4. Change in $\varepsilon$ from transducer 1 to transducer 6 for Observations A through E. The value highlighted in gray is associated with the change in backscatter measured in Figures 11a and 11b.

\begin{tabular}{|c|c|c|c|}
\hline Observation & $\boldsymbol{\varepsilon}$ transducer $\mathbf{1}$ & $\boldsymbol{\varepsilon}$ transducer $\mathbf{6}$ & Factor of increase in $\boldsymbol{\varepsilon}$ \\
\hline $\mathrm{A}$ & $4.8 \times 10^{-5}$ & $2.5 \times 10^{-4}$ & 5 \\
\hline $\mathrm{B}$ & $1.6 \times 10^{-5}$ & $3.9 \times 10^{-5}$ & 2 \\
\hline $\mathrm{C}$ & $4.9 \times 10^{-5}$ & $2.4 \times 10^{-4}$ & 5 \\
\hline $\mathrm{D}$ & $1.9 \times 10^{-4}$ & $3.5 \times 10^{-4}$ & 2 \\
\hline $\mathrm{E}$ & $9.3 \times 10^{-4}$ & $4.0 \times 10^{-3}$ & 5 \\
\hline
\end{tabular}

The backscatter from the waves in Observations B through E is also consistent with backscatter from homogeneous isotropic stratified turbulence in the viscous-convective subrange (see Appendix 1). Using the same assumptions and analysis techniques as in Observation A, it is found $\varepsilon$ increases from transducer 1 to transducer 6 for Observations B through E as well (Table 4). In other words $\varepsilon$ is always larger downstream in Observations B through E. Typically the waves from Observations B through E have stopped growing or are growing slower than they initially were once they reach transducer 6 (Figure 9). Therefore it can be concluded, $\varepsilon$ is increasing as the growth rate is decreasing for Observations B through E as well. 


\section{Discussion}

Estimates of $R i_{o}$

The profile data do not provide adequate resolution to obtain reliable estimates of $\mathrm{Ri}_{\mathrm{o}}$.

Moreover, the time-dependence of the gradients due to ambient perturbations as well as the shear instabilities themselves complicated the estimation of $\mathrm{Ri}_{\mathrm{o}}$. Therefore indirect methods were employed, based on the observed structure and growth rates of the instabilities, expectations from theoretical and numerical and laboratory studies in which $\mathrm{Ri}_{\mathrm{o}}$ was accurately known. The observations presented here offer two ways to estimate $R i_{o}$ based on the two-dimensional structure and evolution of the observed shear instabilities. Method one will be termed the growth rate method. The first method uses the relationship between $R i_{o}$ and the exponential growth rate of shear instabilities from linear stability theory. The growth rate of the waves is measured using the array enabling $R i_{o}$ to be estimated from linear stability theory (Hazel, 1972). The exponential growth rate is estimated by fitting an exponential curve to the first three points in the $h$ vs. $t / \tau$ curves in Figure 9.

The second method uses empirical results from numerical and laboratory studies to estimate $R i_{o}$ using the slope $\left(h_{\max } / \lambda\right)$ of the waves. The data from Figure 3 in Thorpe [1973] and Figure 13a in Wroblewski et al. [2007] is used to create a plot of $R i_{o}$ vs. $h_{\max } / \lambda$ (Figure 12). A line of best fit is calculated for this data, and $R i_{o}$ is estimated for the observational periods $\mathrm{A}$ through E using the relationship derived from the line of best fit (Figure 12).

Both methods result in similar estimates of $R i_{o}$ (Table 5). This suggests that these estimates may be representative of $R i_{o}$ for the shear instabilities in Observations A through E. However, the variability of the slopes and the growth rate are not captured in these estimates. The results are particularly encouraging because the estimates are consistent despite the use of 
different combinations of measured parameters, assumptions about the validity of linear theory for field observations and empirical results to make the estimates.

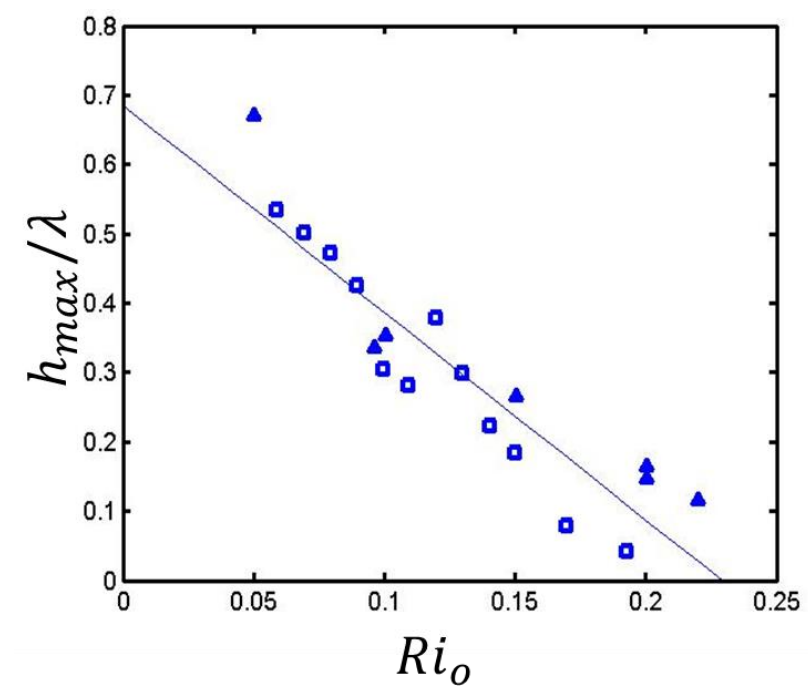

Figure 12. Relationship between wave slope and $R i_{o}$ from Thorpe [1973] and Wroblewski et al. [2007] and references therein. The solid line is the line of best fit. Squares are data from Thorpe [1973] and the triangles are data from Wroblewski et al. [2007].

Table 5. Results of $R i_{o}$ calculations from both methods for the five stratified shear instabilities observed.

\begin{tabular}{|c|c|c|c|c|c|}
\hline Observation & A & B & C & D & E \\
\hline Ri Growth Rate & 0.17 & 0.16 & 0.13 & 0.19 & 0.10 \\
\hline $\boldsymbol{R i}$ Slope & 0.15 & 0.17 & 0.12 & 0.17 & 0.14 \\
\hline
\end{tabular}

Large scale forcing of shear instabilities from Observation A

The forcing which leads to subcritical Ri and then shear instability is considered. On average $R i>0.25$ in the Connecticut River but perturbations in the flow lead to localized occurrence of $R i<0.25$, generating instability. Evidence suggests that the perturbation mechanism for Observation A (Figure 4) is likely a long internal wave propagating downstream. A long undulation of the pycnocline lasting many tens of minutes is observed in the acoustic images from the array (Figure 13), suggesting the presence of an internal wave. The fluid in the upper layer is moving downstream at $\sim 1.2 \mathrm{~m} / \mathrm{s}$. The phase speed of the internal wave is calculated to be $\sim 0.5 \mathrm{~m} / \mathrm{s}$ downstream by assuming a two layer, sheared and inviscid system 
[Sutherland, 2010]. The internal wave phase velocity induces a relative velocity of $\sim 0.7 \mathrm{~m} / \mathrm{s}$

between the internal wave and the fluid in the upper layer.

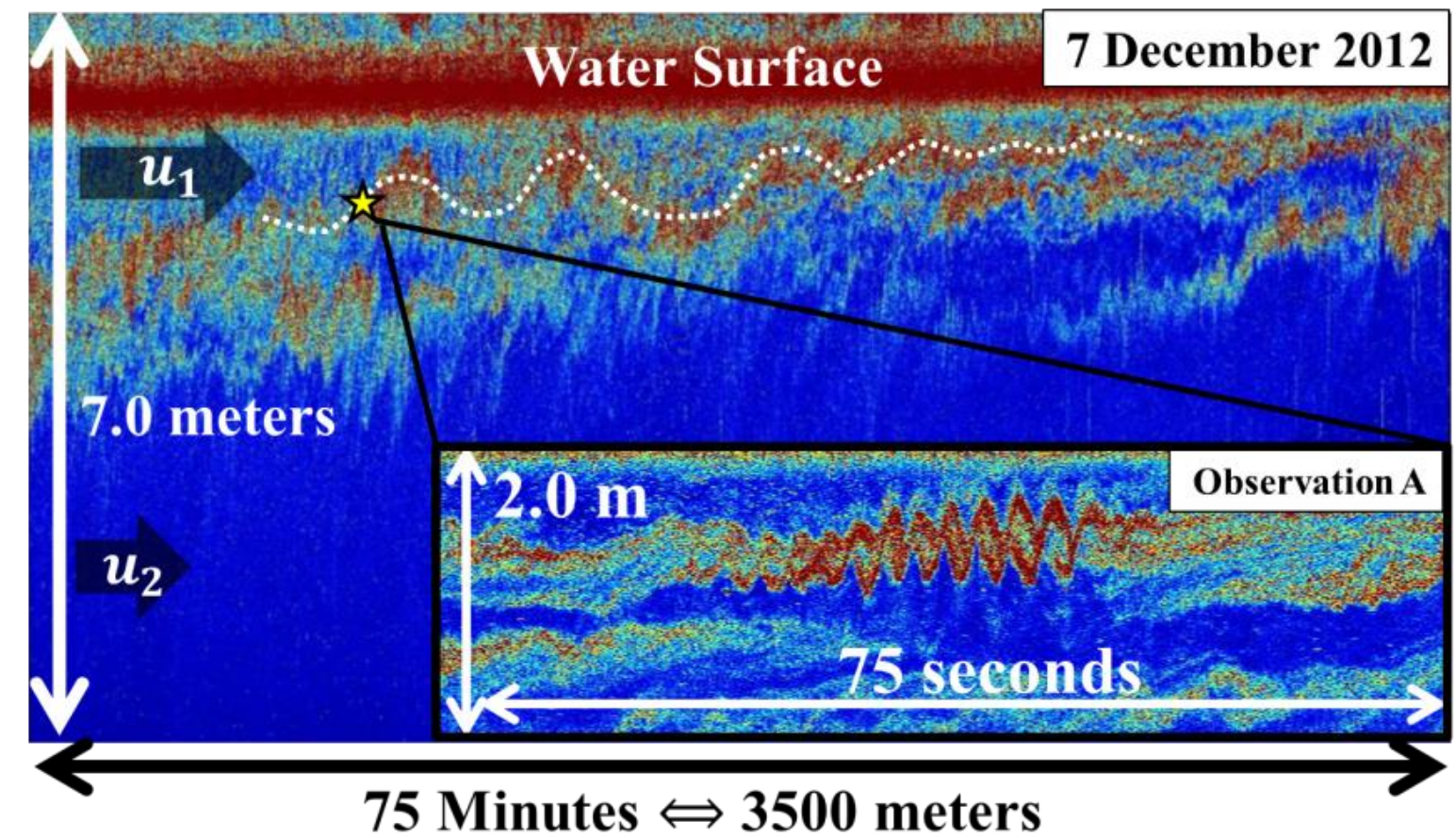

Figure 13. Acoustic image from transducer 1 on 7 December 2012. The white line is meant to illustrate the internal waves along the tilted pycnocline. Note if the $\mathrm{x}$ axis were transformed to space units it would $\sim 3 \mathrm{~km}$ so the interface appears much steeper than it really is. The star indicates the location of the observed stratified shear instability from Observation A.

The internal wave increases the shear across the pycnocline by constricting the fluid in the upper layer, causing an advective acceleration as the fluid in the upper layer passes by the internal wave due to the relative velocity. The increased shear likely decreases $R i$ below 0.25 and the flow becomes unstable. $R i$ is calculated as a function of distance from the trough of the internal wave (Figure 14). This calculation indicates it is possible that the internal wave provides enough of a constriction to decreases $R i$ (Figure 14). The location of the shear instability associated with Observation A within the internal wave (yellow star in Figure 13) is where one would expect the largest advective acceleration of the upper fluid. Additionally, there is an increase in the backscatter intensity at the crests of the internal wave, consistent with the 
hypothesis that internal waves are providing large scale forcing for mixing. This hypothesis cannot be completely validated with these observations because the ADCP could not sample the upper fluid layer as the wave crests of the long internal wave passed by the array due to interference from the surface. It appears that internal waves during this part of the ebb tide could be important in initiating mixing in the Connecticut River estuary.

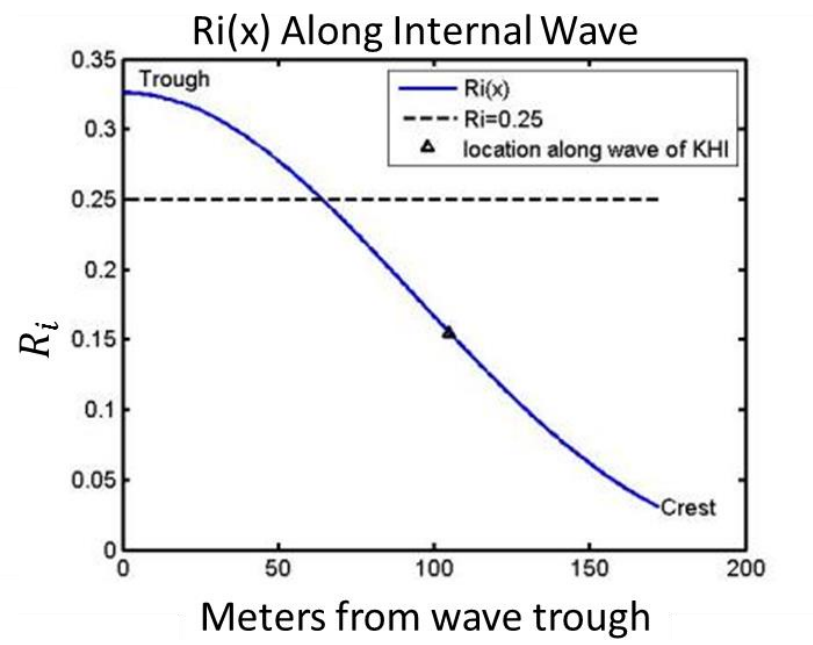

Figure 14. $R i$ as function of distance from the trough of an internal wave with amplitude and wavelength consistent with Observation A . It can be seen that a small constriction caused by the wave can result in a significant decrease in $R i$ in this system. The triangle indicates the location of the shear instability in Observation A. Note the model assumes an inviscid flow so in reality $R i$ probably reaches a minimum below 0.25 before the crest is reached and then increases as a result of turbulent mixing of mass and momentum. The model is meant to demonstrate the internal wave could provide enough of a constriction to decrease $R i$.

\section{Observations compared to Corcos and Sherman [1976]}

A comparison of the evolution of the shear instabilities from Observation A with the model developed by Corcos and Sherman [1976] shows the model and the observations are consistent with each other (Figure 15). The Corcos and Sherman [1976] model predicts the twodimensional evolution of shear instabilities at high $R e$. More specifically, the model predicts that the majority of mixing by the waves at high Reynolds number occurs in the braids of the instabilities and that the growth time can take on the order of 10 shear time scales. In Observations A through E, the strongest stratified turbulence is in the braids, consistent with the predictions of Corcos and Sherman [1976]. It can also be seen that the time scales of growth and 
final amplitude of waves from Observation A are broadly consistent with the model predictions, however, it appears it takes less time for the shear instabilities to arrest their growth in the field than predicted by the model (Figure 15). The most notable aspect of this figure is it shows the model predicts arrest of wave growth faster than what is seen in numerical and laboratory studies and similar to what is observed in Observation A.

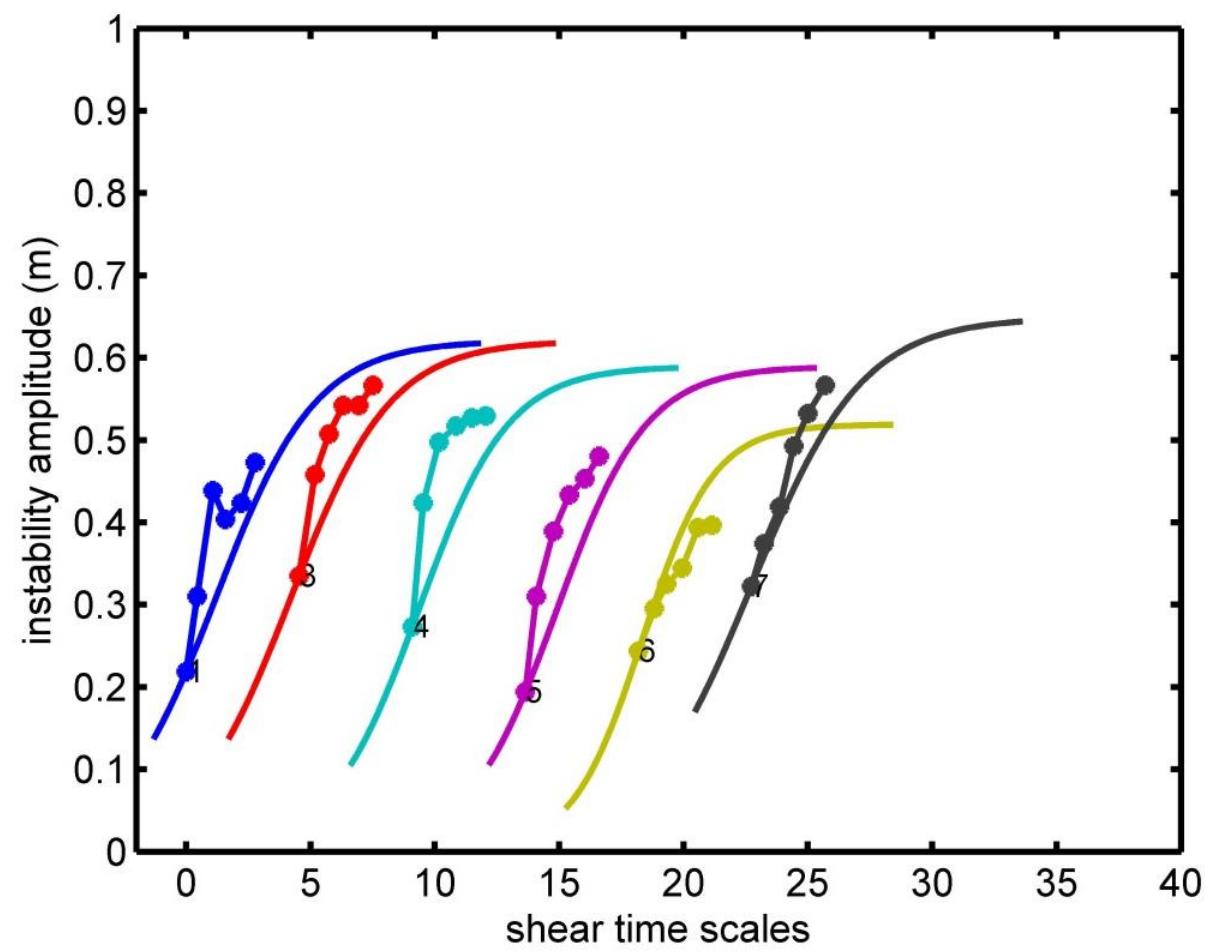

Figure 15. Model predictions of shear instability amplitude from Corcos and Sherman (1976) plotted with the amplitude of stratified shear instabilities from Observation A showing rough agreement in the time scale of the growth phase.

The Corcos and Sherman [1976] model also predicts that the growth of waves is arrested by the secondary shear instabilities on the braids. The consistency between the model and the observations presented here (Figure 15) suggests this prediction is accurate at high $R e$. The finding that $\varepsilon$ increases as the growth rate of the shear instabilities decreases additionally supports the prediction by Corcos and Sherman [1976] that secondary instabilities in the braids limits growth. 


\section{Evolution time scale using energy budget}

The entire life cycle of a shear instability is not observed; however, using a simple box model and the observations of $\varepsilon$, the life time of the waves is estimated and further insight into the plausibility of secondary instabilities arresting the growth of a wave is gained. The model uses prescribed initial and final linear velocity and density profiles (Figure 16). It is assumed that $R i_{o}$ is 0.15 based on the estimates presented in Table 4 and the final $R i$ is 0.3 based on empirical results from laboratory and field experiments [Thorpe, 1972; Koop and Browand, 1976; Geyer and Smith, 1987]. Inserting values of velocity and density from Observation A into the model results in a total energy loss of $2.6 \mathrm{Joules} / \mathrm{m}^{3}$. Using $\varepsilon=1.4 \times 10^{-4} \mathrm{~m}^{2} / \mathrm{s}^{3}$, measured at transducer 6 during Observation A, it is estimated it would take 8 shear time scales for turbulence to dissipate the energy and reach the final state in Figure 16.

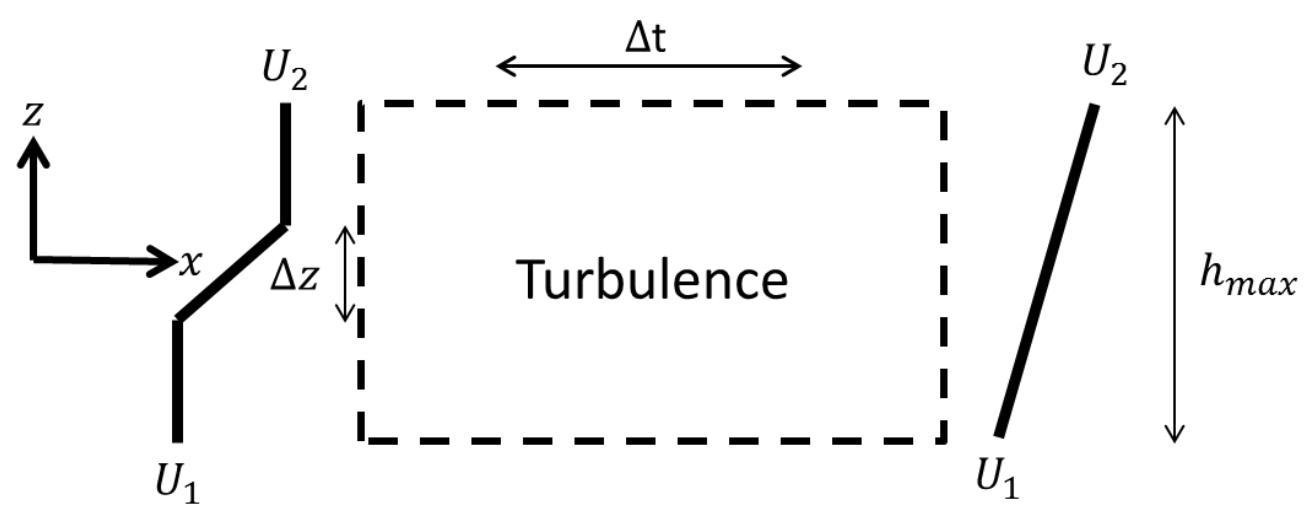

Figure 16. Schematic of the box model used to estimate total energy dissipation. The ambient velocities are $U_{1}$ and $U_{2}$ and $\Delta t$ is the time it takes for the profile on the left to become the profile on the right. It is assumed the velocity is constant above and below linear profile on the right hand side.

It is likely that 8 shear time scales is a reasonable estimate of the entire life of a shear instability and supports the idea that turbulence in the braids of the waves arrests their growth. The dissipation rate is estimated after approximately 5 shear time scales of evolution so some of the energy in the shear layer has already been extracted by the two dimensional part of the flow, 
lifting of fluid and dissipation. Therefore the estimated dissipation rate of $1.4 \times 10^{-4} \mathrm{~m}^{2} / \mathrm{s}^{3}$ in the braid could extract the remaining energy from the shear layer in fewer than 8 shear time scales which is consistent with the abrupt arrest of growth seen in Observations A through E (Figure 9).

The temporal mean of $\varepsilon$ throughout the lifecycle of shear instability is likely lower than $1.4 \times 10^{-4} \mathrm{~m}^{2} / \mathrm{s}^{3}$ in this case. A smaller $\varepsilon$ results in a larger estimate for the lifetime of a shear instability. Therefore, the lifetime of these shear instabilities is likely on the order of 10 shear time scales.

\section{Comparison with lab and numerical experiments}

Differences regarding the evolution and structure of shear instabilities are observed between observations in the Connecticut River estuary at high $R e$ and laboratory and numerical observations at lower $R e$. These differences are present in all five Observations (Observations A through E) and further indicate consistency between these observations and the Corcos and Sherman [1976] model. Further, regardless of the phase of tide, nearly all of the shear instabilities observed in the Connecticut River had the same two-dimensional structures illustrated by the waves from Observation periods A through E. The similarity in structures suggests that all the waves in the Connecticut River evolve in a similar manner to the waves in Observations A through E.

The most outstanding difference between lower $R e$ observations and higher $R e$ observations is the presence of turbulence and mixing in the braids of the waves at high $R e$ as predicted by Corcos and Sherman [1976] and first pointed out by Geyer et al. (2010). Stratified turbulence in the braids is seen in Observations A through E as well (Figures 6, 8 and 1). The location of the turbulence is significant because shear instabilities with highly turbulent cores are 
not observed. In contrast, shear instabilities with highly turbulent cores are a dominating feature in lower Re experiments [Thorpe, 1973; Koop and Browand, 1976; Mashayek and Peltier, 2012; Smyth et al, 2001]. Further, laboratory experiments demonstrate that shear instabilities "roll up" becoming convectively unstable, resulting in turbulent mixing in the cores of the waves. The large scale waves in the Connecticut River do not exhibit "roll up" and collapse to generate turbulent mixing.

Observations A through E also demonstrate that there is a difference in the time scale for the arrest of wave growth between high and low $R e$ observations. In Observations A through $\mathrm{E}$ the waves arrest their growth in 2-4 shear time scales (Figure 9) which is nearly 5-10 times faster than reported by numerical and laboratory experiments [Thorpe, 1973; Koop and Browand, 1979; Mashayek and Peltier, 2013]. The difference is likely a result of both $R e$ effects and the characteristics of the initial perturbation. In terms of $R e$ effects, the discrepancy in growth time appears to be due to shortening of the growth period by the non-linearity introduced by the generation of turbulence on the braids. The turbulence on the braid extracts energy from the two dimensional growth which quickly arrests the growth of the waves and therefore decreases the duration of the growth period.

It is also possible that the total growth period of shear instabilities is shorter at high $R e$ in addition to the time to arrest growth. Scaling analysis based on the observed wavelengths and results from Hazel [1972] show that the shear instabilities originated from and initial shear layer of $0.2 \mathrm{~m}-0.3 \mathrm{~m}$ thick. Many of the shear instabilities observed were initially detected with amplitudes close to these estimated shear layers thicknesses (Figure 9) suggesting the total growth period of the of the shear instabilities in Observations A through $\mathrm{E}$ is on the order of 5 shear time scales. Given that the maximum growth rate scales with the shear rate, this short time 
scale may be explained by the presence of finite amplitude perturbations, in contrast to infitesimal perturbations, which would greatly shorten the timescale for the instabilities to achieve maximum amplitude. Another explanation for the perceived short growth period is the finite length of the array is biasing the interpretation of the data. For example, it is possible only the last portion of the growth phase of the waves is captured with the array and the observations are missing large portions of the initial evolution. Nothing definitive about the total growth period can be deduced from these data, but the data suggest finite amplitude perturbations could be important.

The arrest growth regime seen in Observations A and B could be another difference between lab and numerical experiments. It is difficult to estimate how important this phase of the evolution is because the entire lifecycle of a single wave is not captured with the array. If the arrested growth regime persists for a sizable fraction of the evolution as it appears to in Observation A and B it would represent another large departure from the evolution of shear instabilities at lower $R e$. Based on the present observations it is hard to know if every wave enters this regime for similar amounts of time or if the waves in Observation A and B are the result of a particular condition which enhances the arrested growth regime. A clearer understanding of the arrested growth regime may be found by deploying a longer array of transducers. A longer array of transducers could enable quantification of the entire evolution of shear instabilities resulting in clear observations of the duration of each evolution phase compared to the total evolution time.

\section{Conclusions}

This study demonstrates that a six-channel, calibrated, high-frequency broadband acoustic backscatter system with six upward-looking broadband transducers evenly spaced 1.2 
meters apart along a six-meter long rigid, bottom-mounted steel frame can quantify the evolution of stratified shear instabilities in the field. The array is used to observe the spatial and temporal evolution of the two-dimensional structure of shear instabilities at high $R e$ as well as the turbulence within them.

It is found that the shear instabilities observed in the Connecticut River arrest their growth in 2-4 shear time scales (Figure 9) which is 5-10 times faster than reported by numerical and laboratory experiments [Thorpe, 1973; Koop and Browand, 1979; Mashayek and Peltier, 2013]. The turbulent dissipation rate observed in the braids of the shear instabilities is high enough to arrest wave growth in less than 10 shear time scales based on a simple model for the energy dissipated by shear instability and the resulting stratified turbulence, suggesting the turbulence in the braids arrests the growth of the waves.

The evolution time scale and the presence of turbulence in the braids of the observed shear instabilities are consistent with the model developed by Corcos and Sherman [1976]. Their model predicts that the majority of mixing by shear instabilities occurs in the braids of the shear instabilities, consistent with the strong turbulence detected in the braids of the waves in Geyer et al. [2010] and Observations A through E. The evolution time scales and location of the mixing predicted by the Corcos and Sherman [1976] model are consistent with the shear instabilities observed in this study (Figure 16).

The fast arrest of growth and presence of turbulence within the braids of the waves are attributes of evolution that are not observed in lower Re laboratory and numerical observations of shear instabilities. The lower $R e$ studies are characterized by laminar braids and convective instability in the cores which leads to irreversible mixing and growth arrest times on the order of 20-50 shear time scales [Thorpe, 1973; Koop and Browand, 1979; Mashayek and Peltier, 2013]. 
The existence of an arrested growth regime is observed, which occurs when shear instabilities do not collapse or grow for a period of time nearly equal to the observed growth time of other waves. The arrested growth regime is also consistent with the Corcos and Sherman [1976] model. Their model predicts that shear instability growth is arrested by the dissipation in the braid, which could result in the waves maintaining a coherent structure with little change in amplitude. Unfortunately observations of single shear instability could not be made for more than approximately 5 shear time scales so it is difficult to estimate the significance of the arrested growth regime.

Insights into $R i_{o}$ and the role of internal waves in generating shear instabilities are gained from these observations. In one instance a long internal wave appears to generate an enhanced shear across the pycnocline by creating a constriction between the surface of the river and the pycnocline. This mechanism is likely significant only during the early portions of the ebb tide when the amplitude of long internal waves is a significant fraction of the upper freshwater layer in the estuary.

Using information about the growth rate and two-dimensional structure of shear instabilities, $R i_{o}$ is estimated to be less than 0.2 in most cases and is likely between 0.1 and 0.2 for the shear instabilities observed. Two methods utilizing different assumptions and independent of observational data are used to estimate $R i_{o}$, and both methods agree fairly well. Unfortunately there is no way to validate the estimates due to the vertical resolution of the ADCP and the lack of co-location of the CTD and ADCP.

Further study of stratified shear instability is needed to address a number of first order questions. The extent of the cross flow coherence of stratified shear instabilities and what 
regulates that length scale are parameters that are not understood and could provide great insight into the "patchiness" of turbulence. A more detailed understanding of the initial conditions of stratified shear flow must be addressed as well. $R i_{o}$ was not directly estimated in this study. Nor were the measurements adequate to determine whether the shear layer is turbulent or laminar or if finite amplitude or infinitesimal perturbations initiate the growth of shear instabilities. The potential range of initial conditions and their effects on mixing should be quantified. In terms of making observational progress in some of these areas in the ocean, utilizing acoustic techniques in combination with traditional in-situ measurements is likely to be a fruitful approach. Acoustic techniques are promising because they provide rapid, pervasive and high resolution observations of stratified turbulence parameters in addition to flow visualization. 


\section{Bibliography}

Batchelor, G. K. (1959) "Small-scale Variation of Convected Quantities like Temperature in Turbulent Fluid Part 1. General Discussion and the Case of Small Conductivity." Journal of Fluid Mechanics 5.01: 113.

Carpenter, J. R., Tedford, E. W., Heifetz, E. \& Lawrence, G. A. (2013) "Instability in stratified shear flow: Review of a physical interpretation based on interacting waves" Appl. Mech. Rev. 64(6), 060801-17.

Corcos, G. M., and F. S. Sherman (1976) "Vorticity concentration and the dynamics of unstable free shear layers” J. Fluid Mech., 73, 241-264,doi:10.1017/S0022112076001365.

Chu, D., and T. K. Stanton (1998) "Application of pulse compression techniques to broadband acoustic scattering by live individual zooplankton” J. Acoust. Soc. Am. 104, 39-55.

Fernando, H. J. (1991) “Turbulent mixing in stratified fluids.” Annual review of fluid mechanics, 23(1), 455-493.

Fritts, D. C., G. Baumgarten, K. Wan, J. Werne, and T. Lund (2014) “Quantifying KelvinHelmholtz instability dynamics observed in noctilucent clouds: 2.Modeling and interpretation of observations” J. Geophys. Res. Atmos., 119, 9359-9375.

Geyer, W. R. and D. K. Ralston, (2011) "The dynamics of strongly stratified estuaries". In: Treatise on Estuarine and Coastal Science, E. Wolanski and DS McLusky, Eds., vol. 2:37-51. Waltham: Academic Press.

Geyer, W. R., A. C. Lavery, M. E. Scully, and J. H. Trowbridge. (2010) "Mixing by Shear Instability at High Re." Geophysical Research Letters 37.22.

Hazel, P. (1972) “ Numerical studies of the stability of inviscid stratified shear flows" Journal of Fluid Mechanics, 51, pp 39-61.

Ishimaru, A. (1978) "Wave Propagation and Scattering in Random Media" (Academic, New York), Vol. 2.

Koop, C. G., and F. K. Browand (1979) "Instability and turbulence in a stratified fluid with shear" J. Fluid Mech., 93, 135-159.

Kundu, P. K., I. M. Cohen, D. R. Dowling (2012). "Fluid Mechanics" (Academic Press, Waltham, MA), $5^{\text {th }}$ edition.

Lavery, A. C., W. R. Geyer, and M. E. Scully (2013) "Broadband acoustic quantification of stratified turbulence“ J. Acoust. Soc. Am. 134, 40-54. 
Lavery, A. C., D. Chu, and J. N. Moum (2010) "Measurements of acoustic scattering from zooplankton and oceanic microstructure using a broadband echo sounder” ICES J. Mar. Sci., 67, 379-394.

Lavery, A. C., P. H. Wiebe, S. K. Stanton, G. L Lawson, M. C. Benfield N. Copley (2007) "Determining dominant scatterers of sound in mixed zooplankton populations" J. Acoust. Soc. Am. 122, 3304-3326.

Lavery, A.C., R.W. Schmitt, and T.K. Stanton (2003) "High-frequency acoustic scattering from turbulent oceanic microstructure: The importance of density fluctuations" J. Acoust. Soc. Am., 114, 2685-2697.

Mashayek, A. and W. R. Peltier (2013) "Shear-induced mixing in geophysical flows: does the route to turbulence matter to its efficiency?”J. Fluid Mech,725, 216-261.

Mashayek, A., and W.R. Peltier (2012) 'The 'zoo' of secondary instabilities precursory to stratified shear flow transition. Part 1 Shear aligned convection, pairing, and braid instabilities" J. Fluid Mech., vol. 708, pp. 5-44.

Miles, J. W. (1961) “On the stability of heterogeneous shear flows” J. Fluid Mech. 10, 496-508

Morse, P. M., and K. U. Ingard (1968). “Theoretical Acoustics” (Princeton U. P., Princeton, NJ).

Moum, J. N., D. M. Farmer, W. D. Smyth, L. Armi, and S. Vagle (2003)."Structure and generation of turbulence at interfaces strained by internal solitary waves propagation shoreward over the continental shelf," J. Phys.Oceanogr. 33, 2093-2122.

Osborn, T. R. (1980) "Estimates of the Local Rate of Vertical Diffusion from Dissipation" Measurements,” J. Phys. Oceanogr. 10, 83-89.

Seim, H. E., M. C. Gregg (1994) “Detailed observations of a naturally occurring shear instability“ J. Geophys. Res. 99, 10049-10073.

Smyth, W. D. (2003), Secondary Kelvin-Helmholtz instability in weakly stratified shear flow, J. Fluid Mech., 497, 67-98.

Sreenivasan, K. R. (1995) "On the unviversality of the Kolomogorov constant" Phys. Fluids. 7, 2778-2784.

Sutherland B. R. (2010) “Internal Gravity Waves” (Cambridge: Cambridge UP)

Tedford, E. W., J. R. Carpenter, R. Pawlowicz, R. Pieters, and G. A. Lawrence (2009)

"Observation and analysis of shear instability in the Fraser River estuary" J. Geophys. Res., 114, C11006.

Thorpe, S. A. (1973) "Experiments on instability and turbulence in a stratified shear flow" J. Fluid Mech., 61, 731-751. 
Wroblewski E. D., Coté R. O., Hacker M. J., Dobosy J. R. (2006) “Cliff-Ramp Patterns and Kelvin-Helmholtz Billows in Stably Stratified Shear Flow in the Upper Troposphere: Analysis of Aircraft Measurements" American Meteorological Society , 54, 2521-2539.

Wunsch, C., and R. Ferrari "Vertical Mixing, Energy, And The General Circulation Of The Oceans." Annual Review of Fluid Mechanics 36.1 (2004): 281-314. 


\section{Appendix 1: Additional figures}

Additional figures that contain the data from Observations B through E are presented.

The figures are included here to document the details of each packet of shear instabilities. All the Observations come from a similar portion of the ebb tide (Figure 1A1) as discussed previously.

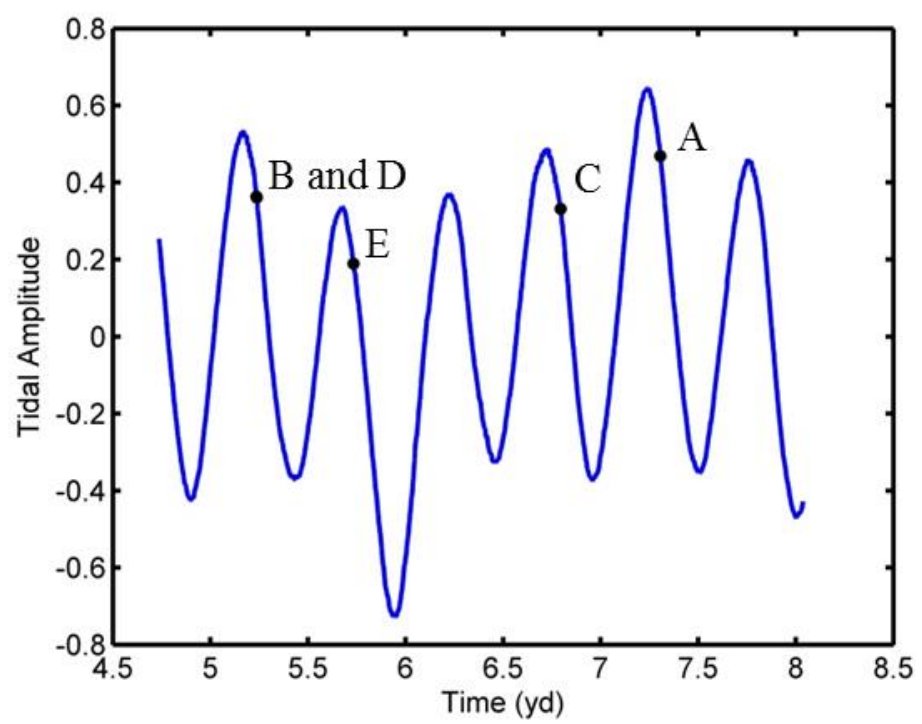

Figure 1A1. Tidal amplitude with the times of Observations A-E marked with dots. All the observations came from the beginning of the ebb tide.

\section{Observation B}

Observation B consists of a packet of four consecutive shear instabilities (Figure 3A1), which occurred on 5 December 2012 at 05:41. A density profile was measured 6.6 minutes before the shear instabilities arrived and a velocity profile was measured $6 \mathrm{~s}$ before the shear instabilities reached the array. The profiles show the environment is sheared and stratified (Figure 2A1). Estimates of $R i$ from the profiles show that a minimum $R i$ occurs in the upper water column between 3 and $5 \mathrm{~m}$ at the same range as the shear instabilities from Observation B (Figure 2A1 (highlighted by the gray box), Figure 3A2). 


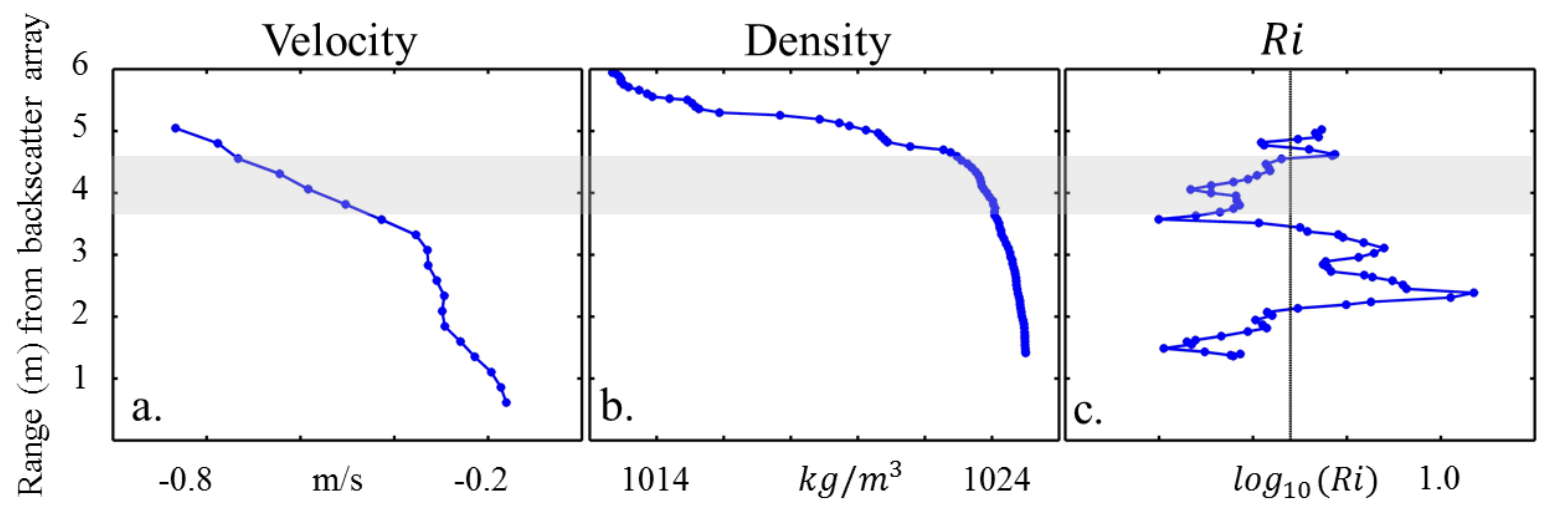

Figure 2A1. Velocity, $R i$, and density profiles for the stratified shear instabilities from Observation B (Figure 3A1). a.) velocity profile obtained from the ADCP on the array, b.) Density profile, inferred from the CTD data. c.) Estimates of $R i$ using the profiles from a.) and b.). The gray line indicates the location of the shear instabilities.

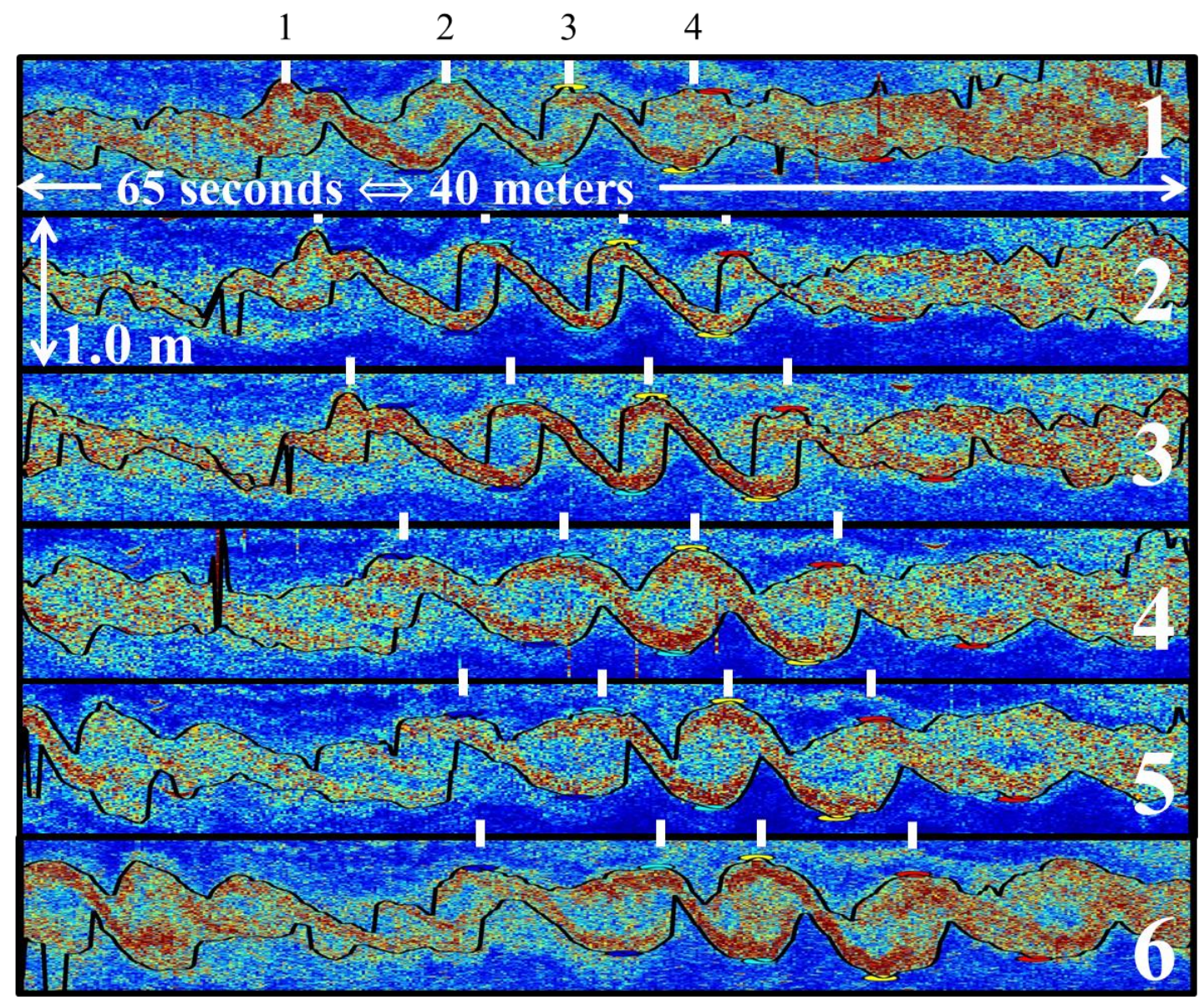

Figure 3A1. Acoustic images from all six transducers on the array for Observation B. The vertical range for each record is $1.0 \mathrm{~m}$ and the horizontal axis is $65 \mathrm{~s}$ in time which corresponds to approximately $40 \mathrm{~m}$ in space using the velocity from the ADCP. The individual stratified shear instabilities are labeled 1-7 at the top of the figure. Most of the shear instabilities grow as they pass from transducer 1 to 6 (labeled on right hand side). Flow is from right to left and the faster fresher water overlies slower, saltier, denser water at the bottom of the images. The black lines are the output of the edge detection algorithm. 
The amplitude of the shear instabilities increases from transducer 1(the most upstream) to transducer 6 (the most downstream) (Figure 3A1). Each shear instability takes approximately 11 $\mathrm{s}$ or 3 shear time scales to advect over the array and reach a final amplitude of $0.75 \mathrm{~m}$ (Figure 3A1). The wavelength of the shear instabilities is $3.3 \mathrm{~m}$ (Table 2).

The backscatter spectrum estimated for Observation B is consistent with homogeneous isotropic stratified turbulence (Figure 4cA1), with a slope of 1.5 (Figure 4cA1), which is broadly consistent with backscatter from homogeneous isotropic stratified turbulence in the viscous convective sub range. It is assumed that the backscatter from all the stratified shear instabilities in Observation B is from homogeneous isotropic stratified turbulence.

a.)

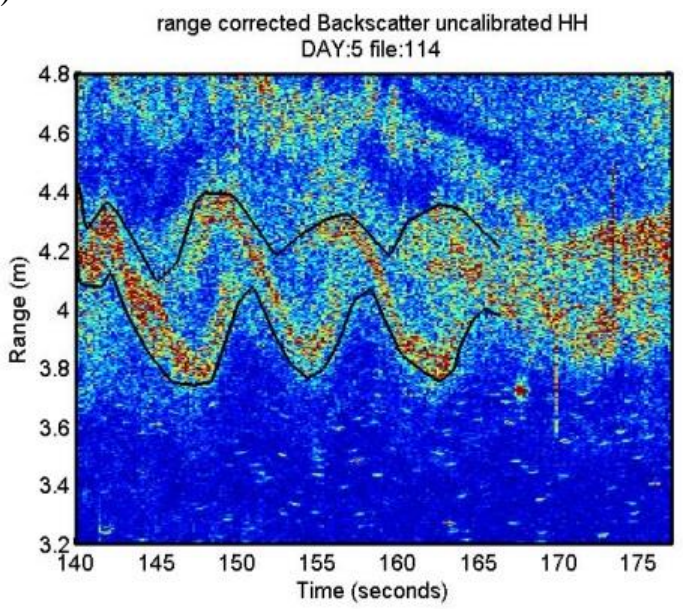

c.)

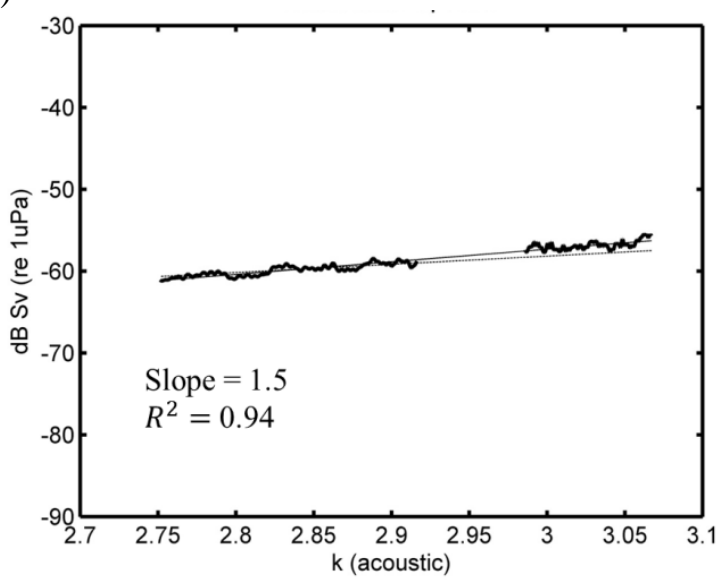

b.)

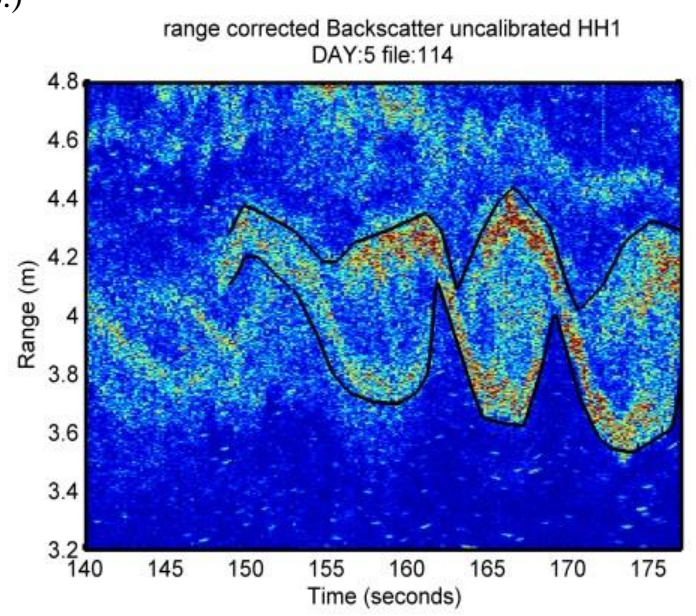

Figure 4A1. Data selected from transducer 1and transducer 6 to make estimates in the change backscatter strength and the backscatter spectrum estimated from transducers 3 and 4. a.) The section of data analyzed from transducer 1 outlined by the black line. b.) The section of data analyzed from transducer 6 outlined by the black line. c.) Result of the spectral analysis: The spectrum is consistent with backscatter from stratified turbulence. Transducers 3 and 4 were used to create this spectrum. 
Figures 4aA1 and 4bA1 show the region of data selected for analysis from transducers 1

and 6. The same stratified shear instabilities are selected in each image. Assuming all other terms in equation 2 are constant, $\varepsilon$ increases by a factor of 5 (Table 3 ).

\section{Observation $C$}

Observation $\mathrm{C}$ consists of a packet of four consecutive shear instabilities (Figure 6A1), which occurred on 6 December 2012 at 19:04. A density profiles was measured 2 minutes before the shear instabilities arrived and a velocity profile was measured $6 \mathrm{~s}$ after the shear instabilities reached the array. The profiles show the environment is sheared and stratified (Figure 5A1). Estimates of $R i$ from the profiles show that a minimum of $R i$ occurs in the upper water column between 3 and $5 \mathrm{~m}$ at the same range as the shear instabilities from Observation $\mathrm{C}$ (Figure 5A1 (highlighted by the gray box), Figure 6A1).

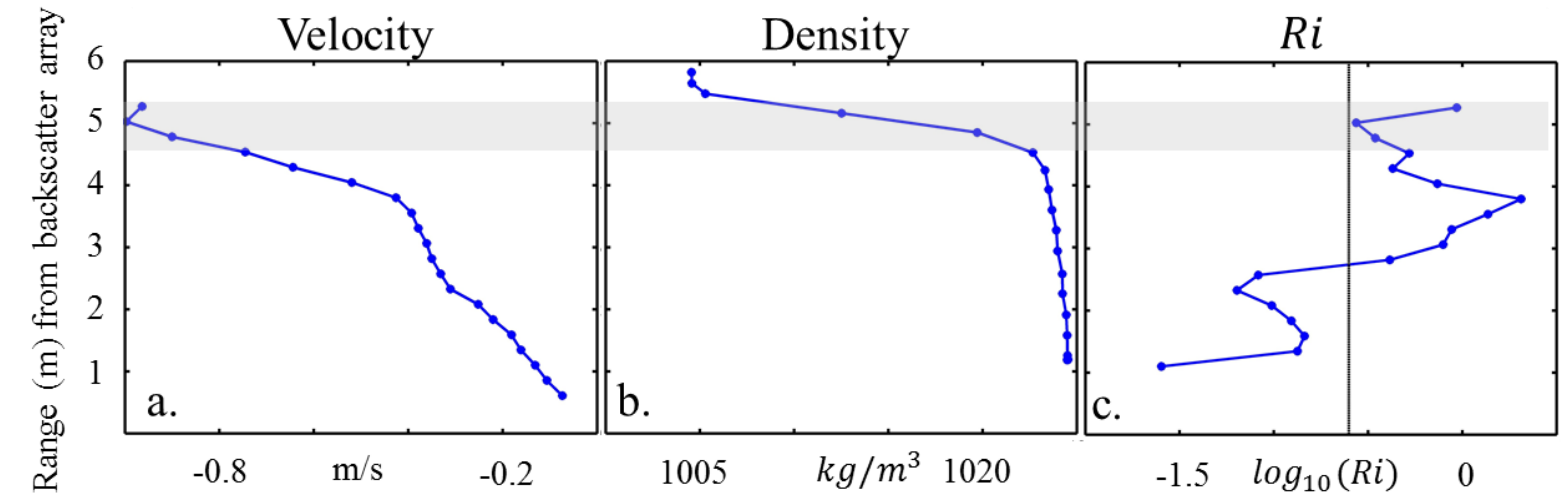

Figure 5A1. Velocity, $R i$, and density profiles for the stratified shear instabilities from Observation C (Figure 6A1). a.) velocity profile obtained from the ADCP on the array, b.) Density profile, inferred from the CTD data. c.) Estimates of $R i$ using the profiles from a.) and b.). The gray line indicates the location of the shear instabilities.

The amplitude of the shear instabilities increases from transducer 1(the most upstream) to transducer 6 (the most downstream) (Figure 6A1). Each shear instability takes approximately $7 \mathrm{~s}$ or 3 shear time scales to advect over the array and reach a final amplitude of $0.6 \mathrm{~m}$ (Figure 2A1). The wavelength of the shear instabilities is $1.7 \mathrm{~m}$ (Table 2). 
The backscatter spectrum is estimated for Observation $\mathrm{C}$ and it demonstrates that the

backscatter is consistent with homogeneous isotropic stratified turbulence (Figure 7cA1). The

slope of the resulting backscatter spectrum is 1.5 (Figure 7cA1). It is assumed that the

backscatter from all the stratified shear instabilities in Observation $\mathrm{C}$ is from homogeneous

isotropic stratified turbulence.

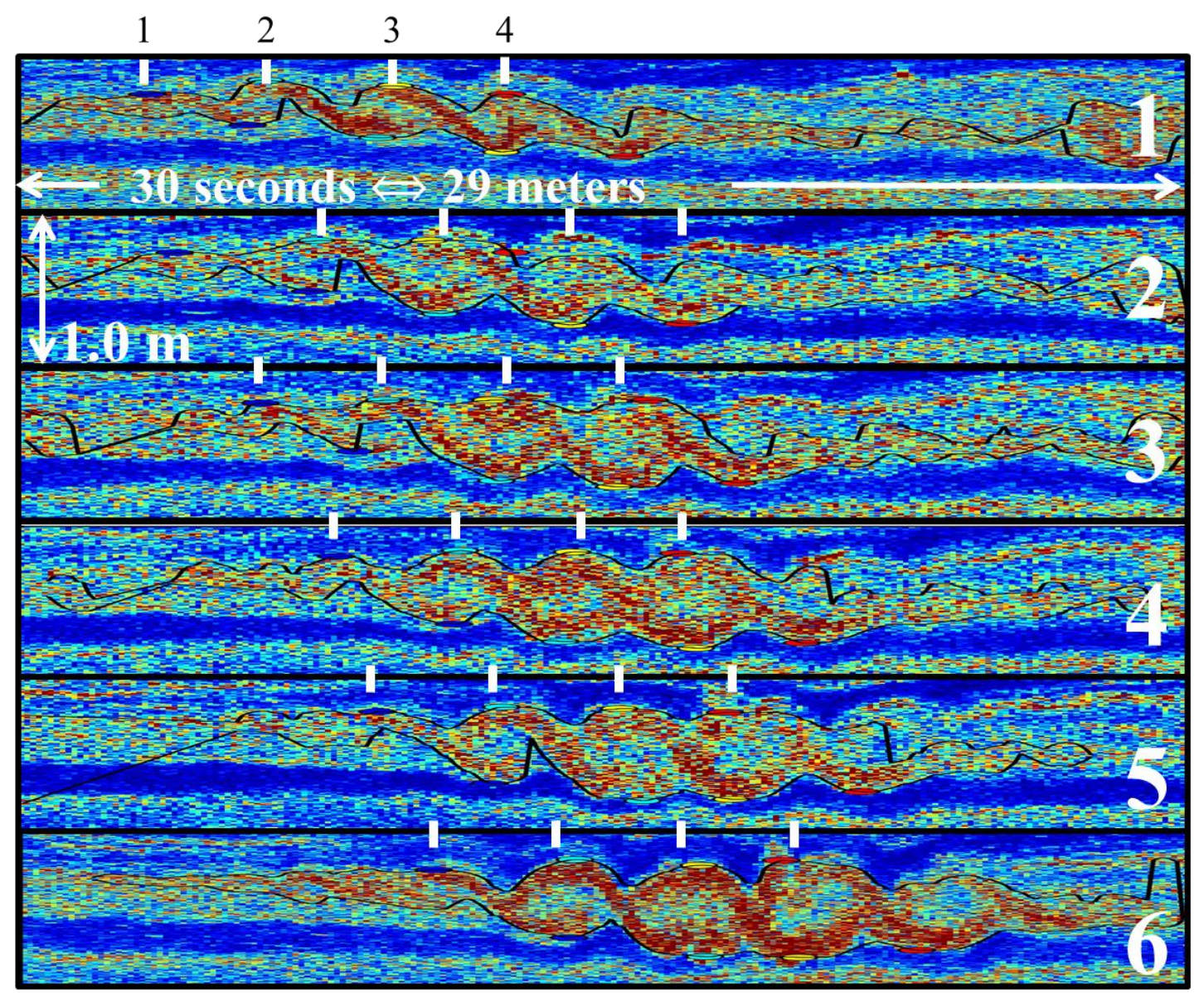

Figure 6A1. Acoustic images from all six transducers on the array from Observation $\mathrm{C}$. The vertical range for each record is $1.0 \mathrm{~m}$ and the horizontal axis is $30 \mathrm{~s}$ in time which corresponds to approximately $29 \mathrm{~m}$ in space using the velocity from the ADCP. The individual stratified shear instabilities are labeled 1-7 at the top of the figure. Most of the shear instabilities grow as they pass from transducer 1 to 6 (labeled on right hand side). Flow is from right to left and the faster fresher water overlies slower, saltier, denser water at the bottom of the images. The black lines are the output of the edge detection algorithm.

Figures $7 \mathrm{aA} 1$ and $7 \mathrm{bA} 1$ show the region of data selected for analysis from transducers 1

and 6. The same stratified shear instabilities are selected in each image. Assuming all other terms in equation 2 are constant, $\varepsilon$ increases by a factor of 5 (Table 3 ). 
a.)

CTRiver_12062012_468 range corrected Backscatter uncalibrated HL DAY:7 end time:19:8:6.5374 EDT yd: 342

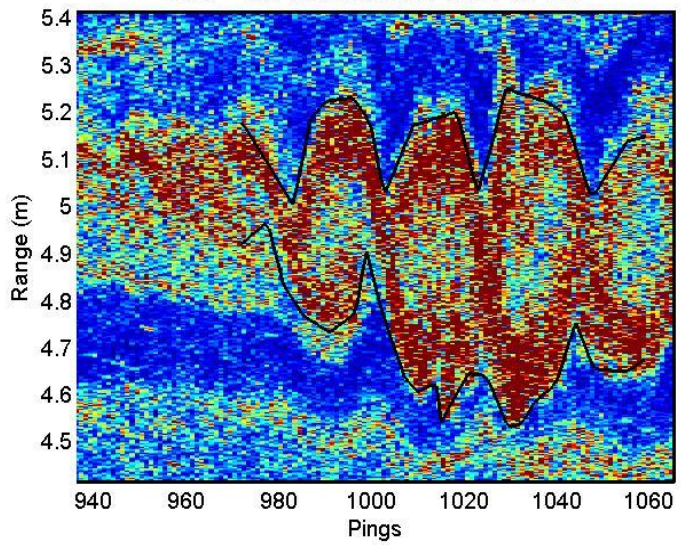

c.)

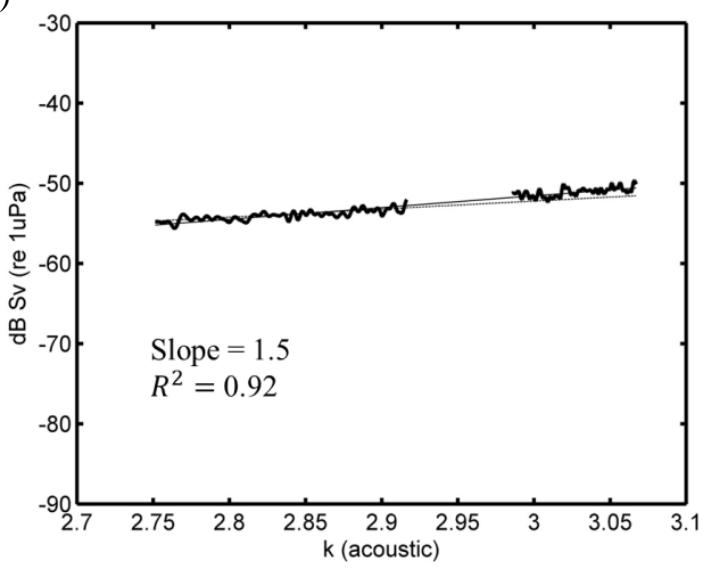

b.)

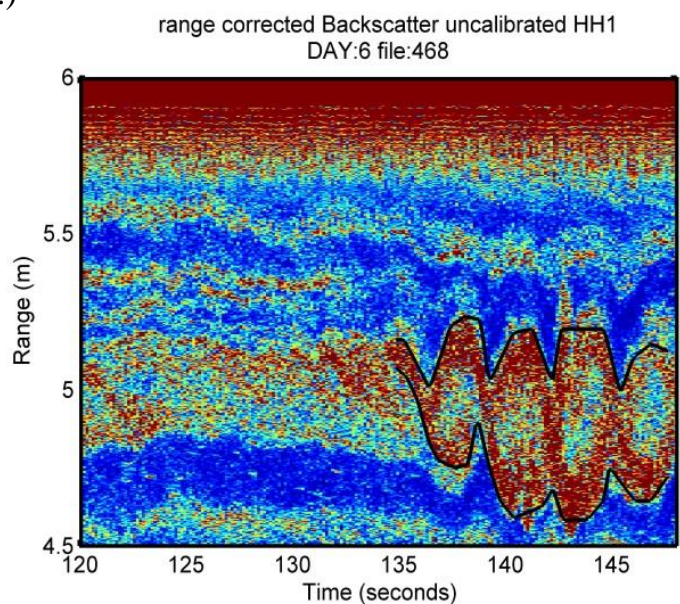

Figure 7A1. Data selected from transducer 1and transducer 6 to make estimates in the change backscatter strength and the backscatter spectrum estimated from transducers 3 and 4. a.) The section of data analyzed from transducer 1 outlined by the black line. b.) The section of data analyzed from transducer 6 outlined by the black line. c.) Result of the spectral analysis: The spectrum is consistent with backscatter from stratified turbulence. Transducers 3 and 4 were used to create this spectrum.

\section{Observation D}

Observation D consists of a packet of four consecutive shear instabilities (Figure 9A1), which occurred on 5 December 2012 at 05:41. A density profiles was measured 7 minutes before the shear instabilities arrived and a velocity profile was measured $3.4 \mathrm{~s}$ before the shear instabilities reached the array. The profiles show the environment is sheared and stratified (Figure 8A1). Estimates of $R i$ from the profiles show that a low value of $R i$ occurs in the top of the water column between 5 and $5.5 \mathrm{~m}$ at the same range as the shear instabilities from Observation D (Figure 8A1 (highlighted by the gray box), Figure 9A1). 


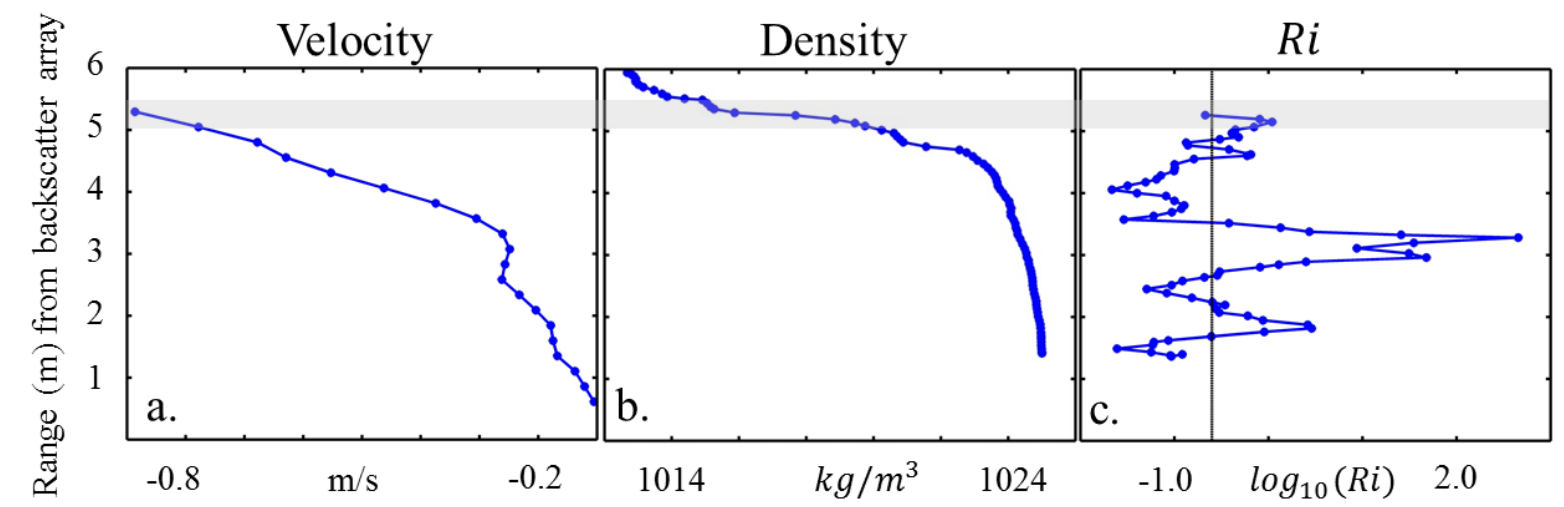

Figure 8A1. Velocity, $R i$, and density profiles for the stratified shear instabilities from Observation D (Figure 9A1). a.) velocity profile obtained from the ADCP on the array, b.) Density profile, inferred from the CTD data. c.) Estimates of $R i$ using the profiles from a.) and b.). The gray line indicates the location of the shear instabilities.

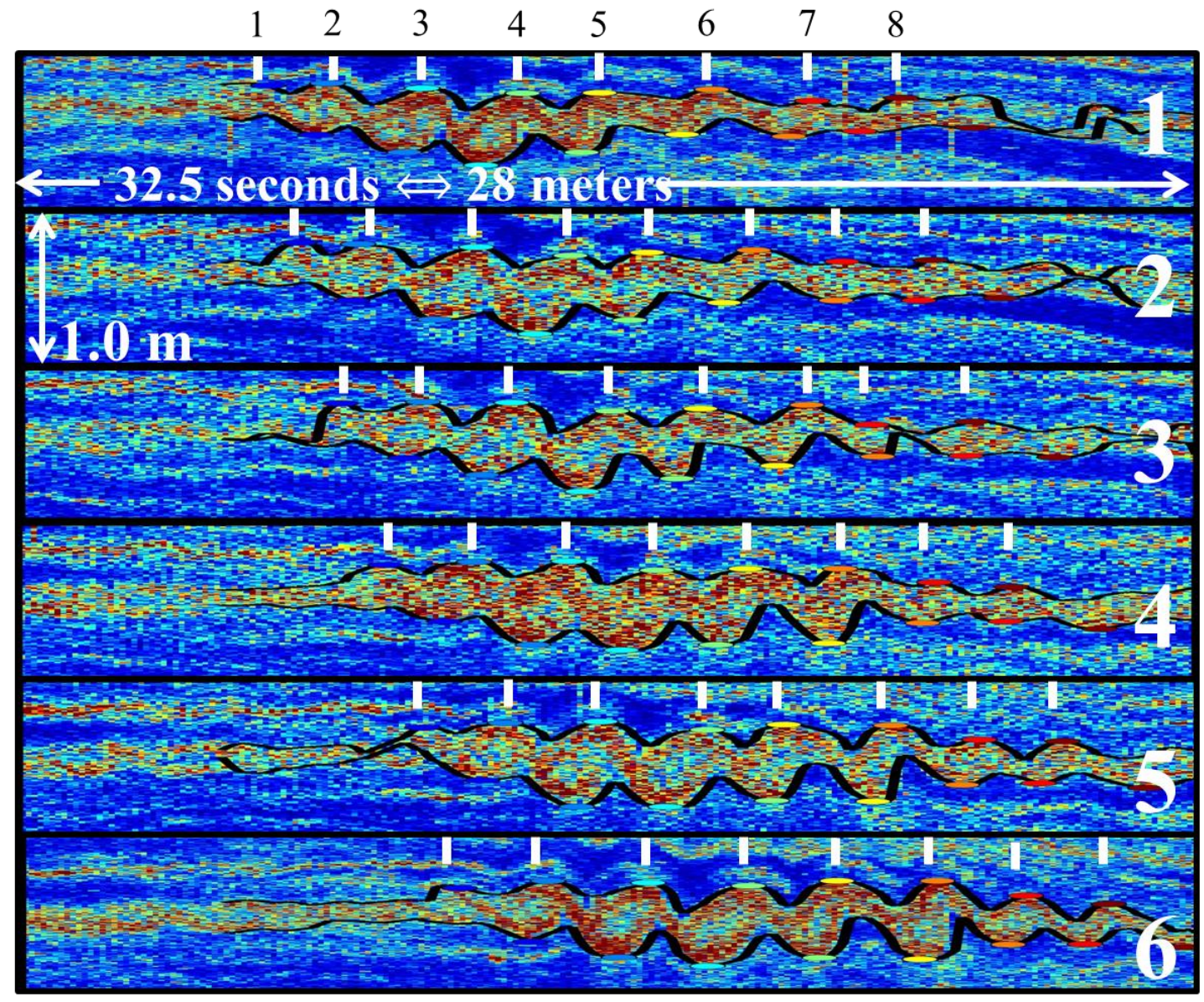

Figure 9A1. Acoustic images from all six transducers on the array from Observation D. The vertical range for each record is $1.0 \mathrm{~m}$ and the horizontal axis is $32.5 \mathrm{~s}$ in time which corresponds to approximately $28 \mathrm{~m}$ in space using the velocity from the ADCP. The individual stratified shear instabilities are labeled 1-7 at the top of the figure. Most of the shear instabilities grow as they pass from transducer 1 to 6 (labeled on right hand side). Flow is from right to left and the faster fresher water overlies slower, saltier, denser water at the bottom of the images. The black lines are the output of the edge detection algorithm. 
The amplitude of the shear instabilities increases from transducer 1(the most upstream) to transducer 6 (the most downstream) (Figure 6). Each shear instability takes approximately $6 \mathrm{~s}$ or 3 shear time scales to advect over the array and reach a final amplitude of 0.6m (Figure 9A1).

The wavelength of the shear instabilities is $2.4 \mathrm{~m}$ (Table 2).

a.)

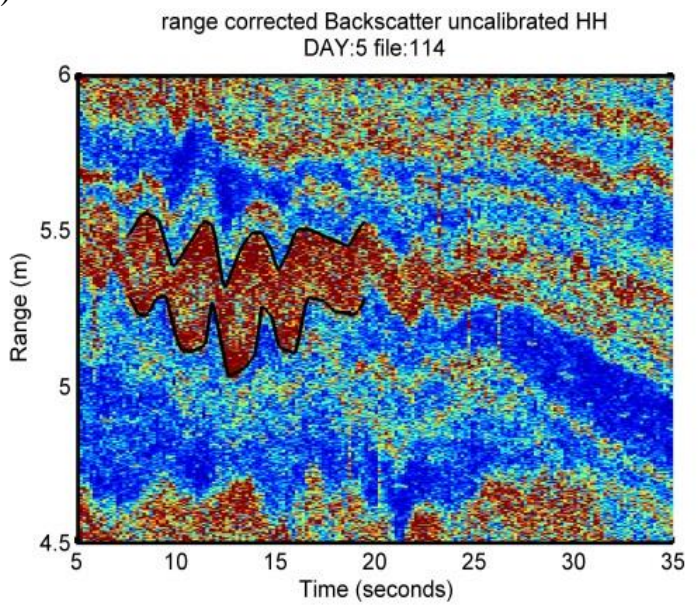

c.)

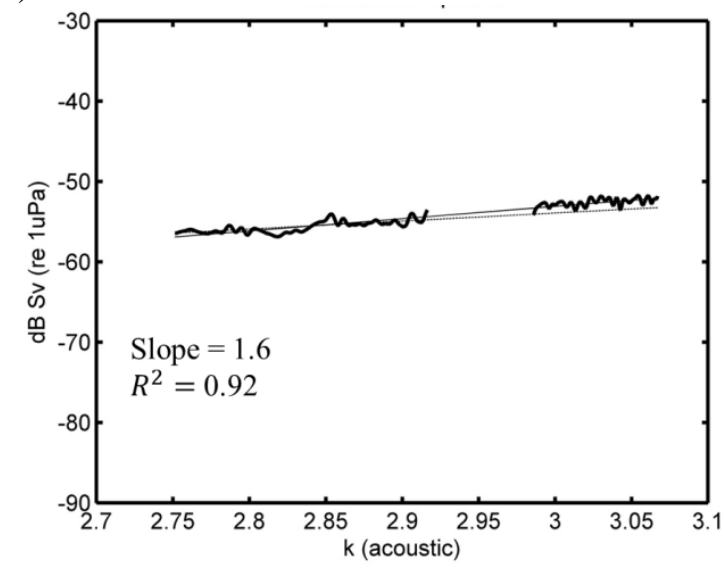

b.)

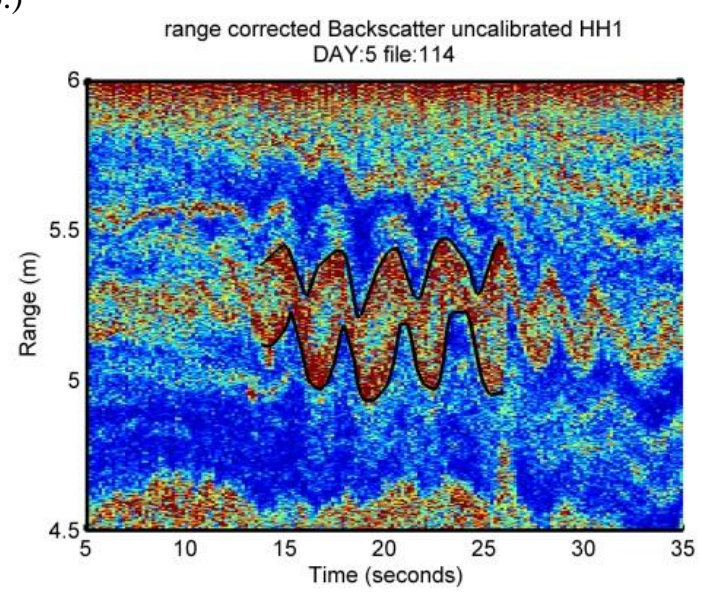

Figure 10A1. Data selected from transducer 1and transducer 6 to make estimates in the change backscatter strength and the backscatter spectrum estimated from transducers 3 and 4. a.) The section of data analyzed from transducer 1 outlined by the black line. b.) The section of data analyzed from transducer 6 outlined by the black line. c.) Result of the spectral analysis: The spectrum is consistent with backscatter from stratified turbulence. Transducers 3 and 4 were used to create this spectrum.

The backscatter spectrum is estimated for Observation D and it demonstrates that the backscatter is consistent with homogeneous isotropic stratified turbulence (Figure 10cA1). The slope of the resulting backscatter spectrum is 1.6 (Figure 10cA1), which is broadly consistent with backscatter from homogeneous isotropic stratified turbulence in the viscous convective sub 
range. It is assumed that the backscatter from all the stratified shear instabilities in Observation D is from homogeneous isotropic stratified turbulence.

Figures 10aA1 and 10bA1 show the region of data selected for analysis from transducers 1 and 6. The same stratified shear instabilities are selected in each image. Assuming all other terms in equation 2 are constant, $\varepsilon$ increases by a factor of 2 (Table 3 ).

\section{Observation E}

Observation E consists of a packet of four consecutive shear instabilities (Figure 12A1), which occurred on 5 December 2012 at 17:35. A density profiles was measured 4.3 minutes before the shear instabilities arrived and a velocity profile was measured $1.8 \mathrm{~s}$ after the shear instabilities reached the array. The profiles show the environment is sheared and stratified (Figure 11A1). Estimates of $R i$ from the profiles show that $R i$ is low throughout water column during Observation E (Figure 11A1 (highlighted by the gray box), Figure 12A1).

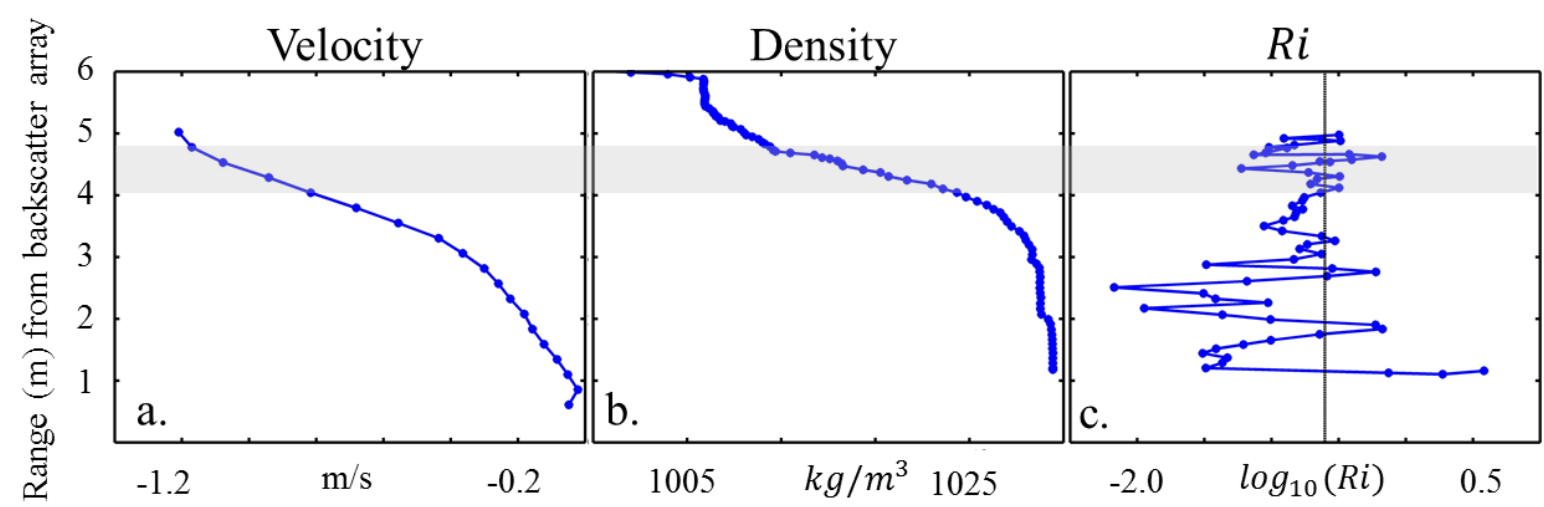

Figure 11A1. Velocity, $R i$, and density profiles for the stratified shear instabilities from Observation E (Figure 12A1). a.) velocity profile obtained from the ADCP on the array, b.) Density profile, inferred from the CTD data. c.) Estimates of $R i$ using the profiles from a.) and b.). The gray line indicates the location of the shear instabilities.

The amplitude of the shear instabilities increases from transducer 1(the most upstream) to transducer 6 (the most downstream) (Figure 6). Each shear instability takes approximately $6 \mathrm{~s}$ or 3 shear time scales to advect over the array and reach a final amplitudes between 0.5 and $0.6 \mathrm{~m}$ (Figure 12A1). The wavelength of the shear instabilities is $1.5 \mathrm{~m}$ (Table 2). 


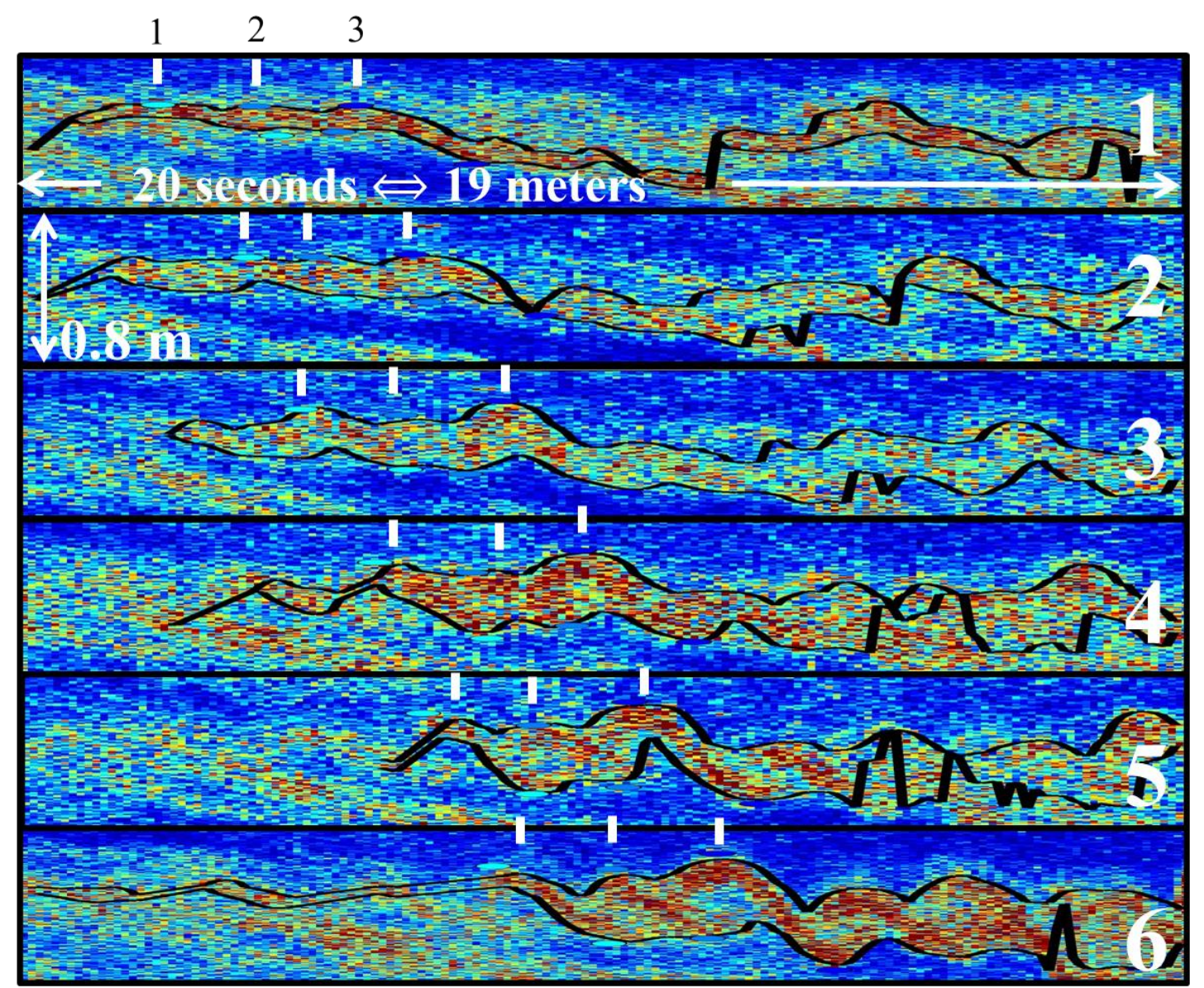

Figure 12A1. Acoustic images from all six transducers on the array from Observation $E$. The vertical range for each record is $0.8 \mathrm{~m}$ and the horizontal axis is $20 \mathrm{~s}$ in time which corresponds to approximately $19 \mathrm{~m}$ in space using the velocity from the ADCP. The individual stratified shear instabilities are labeled 1-7 at the top of the figure. Most of the shear instabilities grow as they pass from transducer 1 to 6 (labeled on right hand side). Flow is from right to left and the faster fresher water overlies slower, saltier, denser water at the bottom of the images. The black lines are the output of the edge detection algorithm.

The backscatter spectrum is estimated for Observation E and it demonstrates that the backscatter is consistent with homogeneous isotropic stratified turbulence (Figure 13cA1). The slope of the resulting backscatter spectrum is 1.8 (Figure 13cA1). It is assumed that the backscatter from all the stratified shear instabilities in Observation $\mathrm{E}$ is from homogeneous isotropic stratified turbulence. 
Figures $13 \mathrm{aA} 1$ and $13 \mathrm{bA} 1$ show the region of data selected for analysis from transducers

1 and 6. The same stratified shear instabilities are selected in each image. Assuming all other terms in equation 2 are constant, $\varepsilon$ increases by a factor of 5 (Table 3 ).

a.)

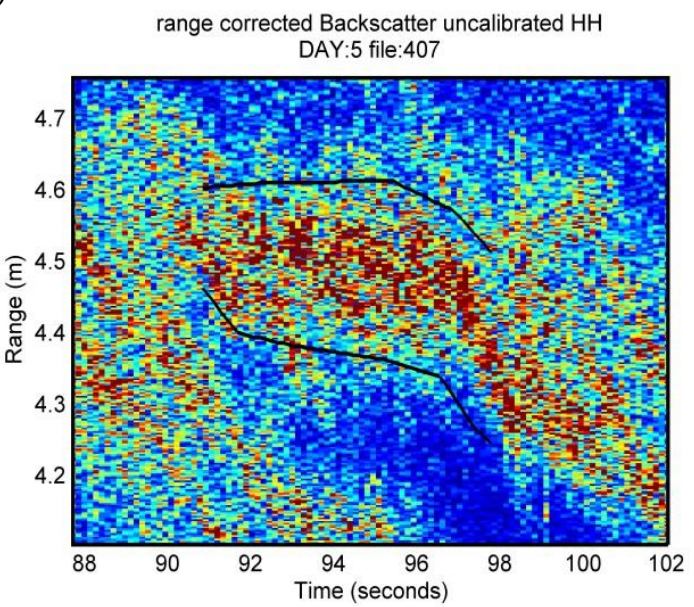

c.)

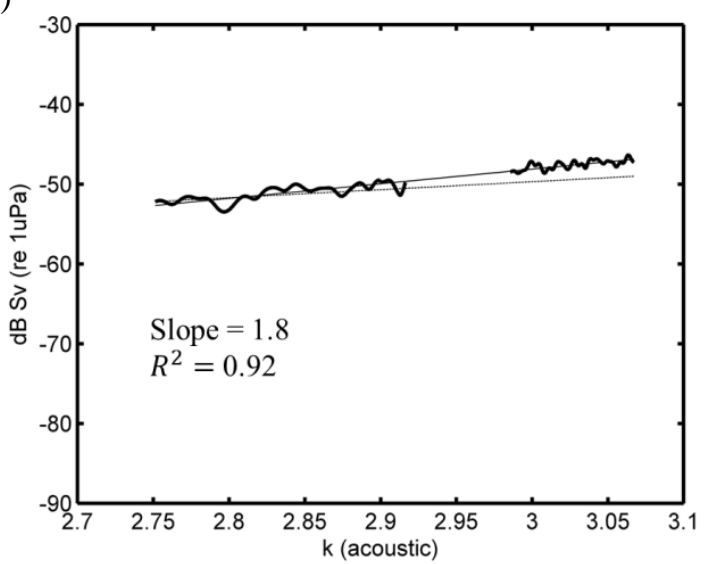

b.)

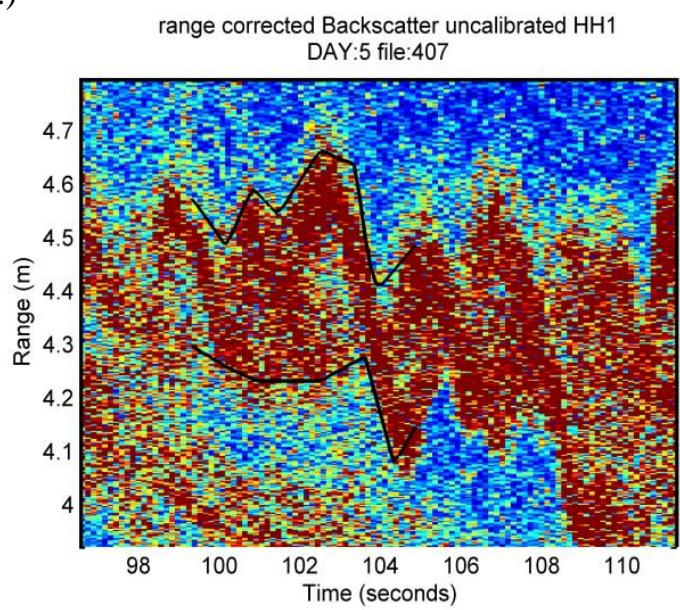

Figure 13A1. Data selected from transducer 1and transducer 6 to make estimates in the change backscatter strength and the backscatter spectrum estimated from transducers 3 and 4. a.) The section of data analyzed from transducer 1 outlined by the black line. b.) The section of data analyzed from transducer 6 outlined by the black line. c.) Result of the spectral analysis: The spectrum is consistent with backscatter from stratified turbulence. Transducers 3 and 4 were used to create this spectrum.

Array alignment with the flow

During the ebb tide the array was typically aligned within 10 degrees of the flow (Figure 14A1). A 10 degree misalignment is fairly small when considering the beam widths of the transducers and ranges of interest in this study. For a 10 degree miss alignment a parcel of water passing through the center point of the array at $5 \mathrm{~m}$ range will be ensonified by all the transducers on the array. Figure 14A1 shows the median alignment of the array with the flow from each velocity profile. Figure $15 \mathrm{~A} 1$ shows the range dependent alignment of the array. It can 
be seen during ebb tides the alignment through the water column is fairly uniform except for very close to the array. During the flood tides the alignment is not as good and can reach as high as 20 degrees out of alignment (Figure 15A1). It can also be seen that the alignment is consistently poor at the location of the velocity maxima during the flood tide (Figure 15A2).

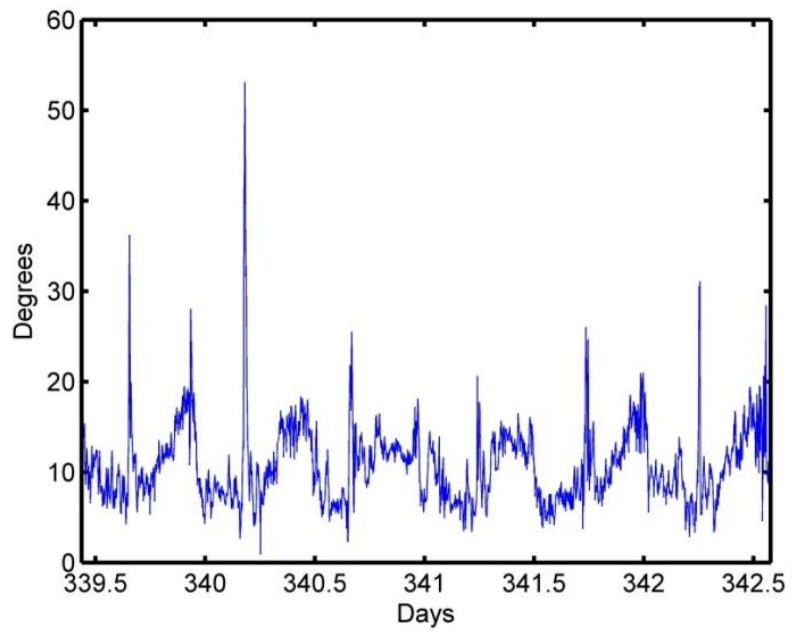

Figure 14A1. Median alignment through the water column of the backscatter array. The array is typically aligned within 10 degrees of the flow during ebb tides and within 15 degrees during flood tides.

a.)

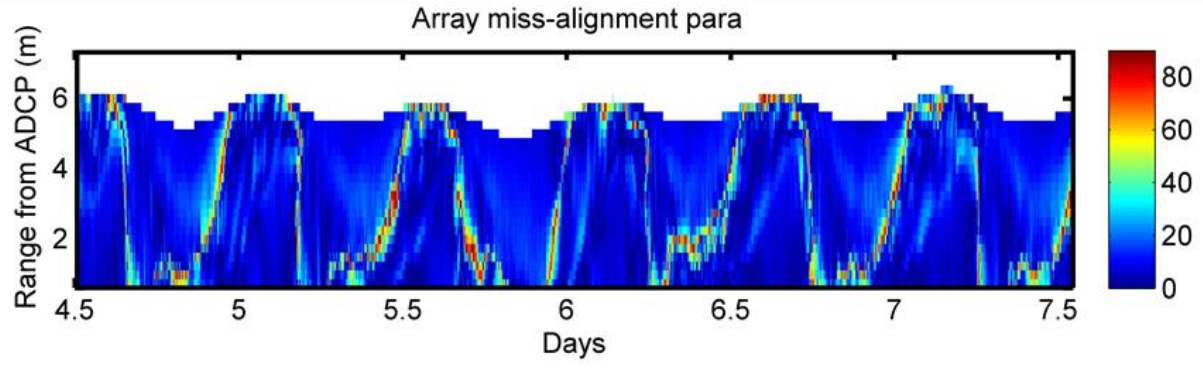

b.)

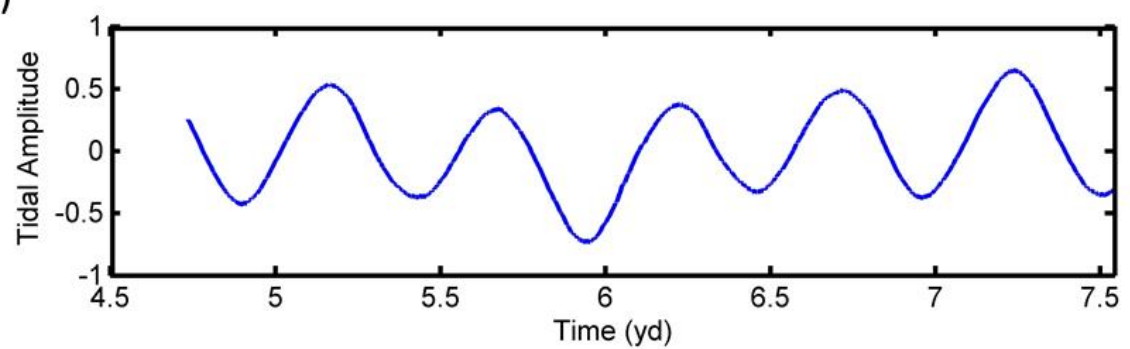

Figure 15A1: a) Array alignment throughout the water column as a function of time and. b) The tidal amplitude during the deployment. a) The alignment of the array is consistent through the water column during ebb tides and substantial miss alignment is detected at the velocity maxima during flood tides. The color axis is in degrees of miss alignment. b) The tidal amplitude demonstrating the connection between array alignment with the flow and the phase of the tide. 


\section{Appendix 2: Additional observations}

The purpose of this section is to briefly characterize the Connecticut River estuary during the experiment for ebb and flood tides using the time series measurements of velocity and density profiles and acoustic backscatter measured at the deployment location (Figure 1). This section is also used to disclose any other interesting observations made during the deployment that could not be further pursued within the scope of this thesis. As mentioned previously velocity profiles from the ADCP were measured every $30 \mathrm{~s}$ and CTD casts were done for 5 minutes four times an hour. The ADCP was mounted on the array and the CTD casts were done from the R/V Connecticut which was situated approximately $200 \mathrm{~m}$ to the west of the array.

\section{ADCP Measurements}

The array was oriented in two different positions during the experiment. The ADCP records for the portions of the deployment where the array was parallel and perpendicular to the flow are presented separately. The array was aligned parallel with the flow for the majority of the five day deployment. During the last day of the experiment the array was aligned perpendicular to the flow to investigate cross-channel variations in the backscatter. The ADCP and CTD data recorded while the array was parallel to the flow will be discussed in detail; the remaining data is shown but not discussed.

\section{The Connecticut River: Ebb tide}

During ebb tides the velocity near the bed of the river is zero or nearly zero and the surface velocity is approximately $1.0 \mathrm{~m} / \mathrm{s}$ (Figure 1A2, Figure 2A2). The velocity at the bed remains nearly zero until the salt wedge and therefore stratification is entirely mixed out of the estuary or the flood tide starts pushing the wedge upriver. If the wedge is expelled from the estuary the flow becomes a homogeneous channel flow. Whether or not the wedge is mixed out 
of the estuary depends on the river discharge, tidal amplitude and bottom friction [Geyer and Ralston, 2011].

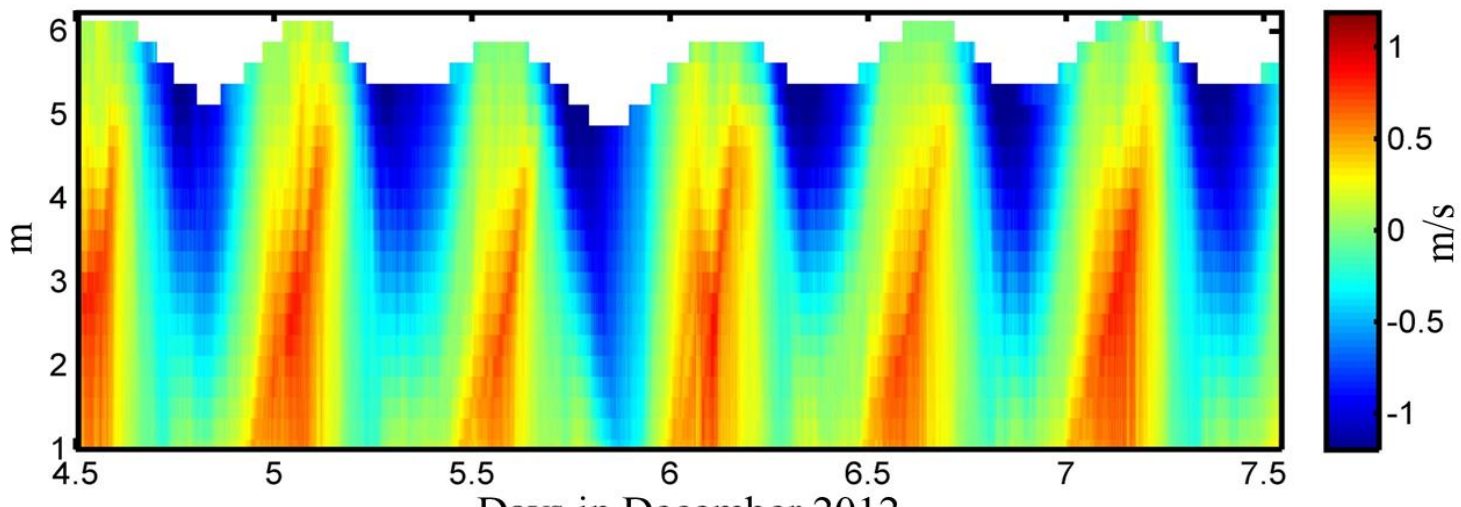

Figure 1A2. Velocity profiles for the along array (along channel) direction during the portion of the deployment where the array was parallel to the flow. The ebb tides are characterized by broadening of the shear layer as the ebb progresses. The flood tide is characterized by a velocity maxima that propagates up through the water column as the flood tide progresses. The color bar is in $\mathrm{m} / \mathrm{s}$ and positive values are upriver.

At the start of the ebb the strongest shear is at the top of the water column which is set up by the arrested advance of the salt wedge and the fresh river water above the wedge flowing out of the estuary. The shear is significant enough to overcome the stable stratification and initiate mixing via shear instability. The observations of shear instabilities presented in this thesis primarily come from the portion of the ebb tide when the shear is in the upper water column. Both density and momentum is mixed by the shear instabilities which results in broadening of the pycnocline (i.e. erosion of the salt wedge) and shear layer. A result of the broadening pycnocline and shear layer is the wavelength and often amplitude of the shear instabilities increases (Figure 4A2). The process of shear layer and pycnocline broadening continues until mid-ebb where the velocity and density profiles become nearly linear through the water column (Figure 1A2, Figure 3A2). At this point in the ebb the mixing becomes most intense [Lavery et $a l, .2013$ ] and shear instabilities reach amplitudes of nearly three meters, occupying nearly $50 \%$ of the water column (Figure 4A2). The mixing continues until either the stratification is gone 
and the flow becomes homogeneous or the tide starts flooding which begins the advance of the salt wedge back up the estuary.

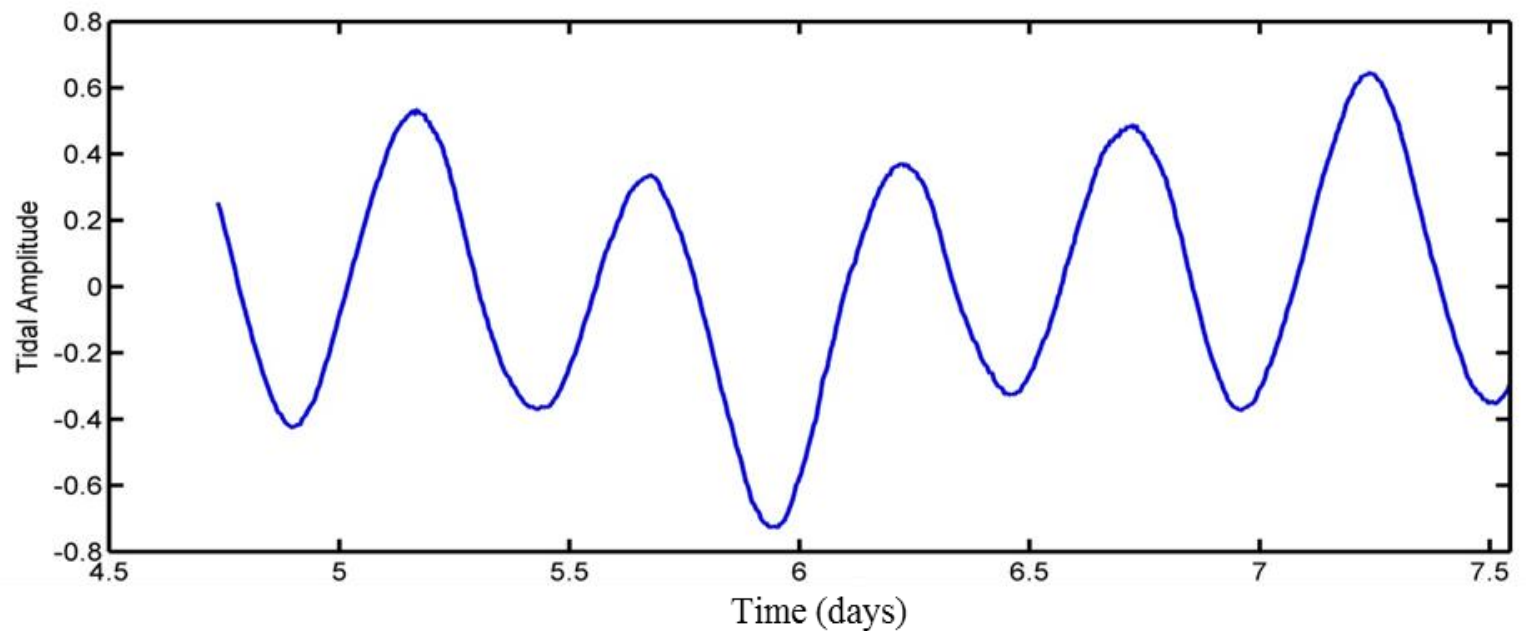

Figure 2A2. Tidal amplitude recorded from the CTD fixed to the array. Note the amplitude of the tidal range varies and cycles with a twelve hour period.

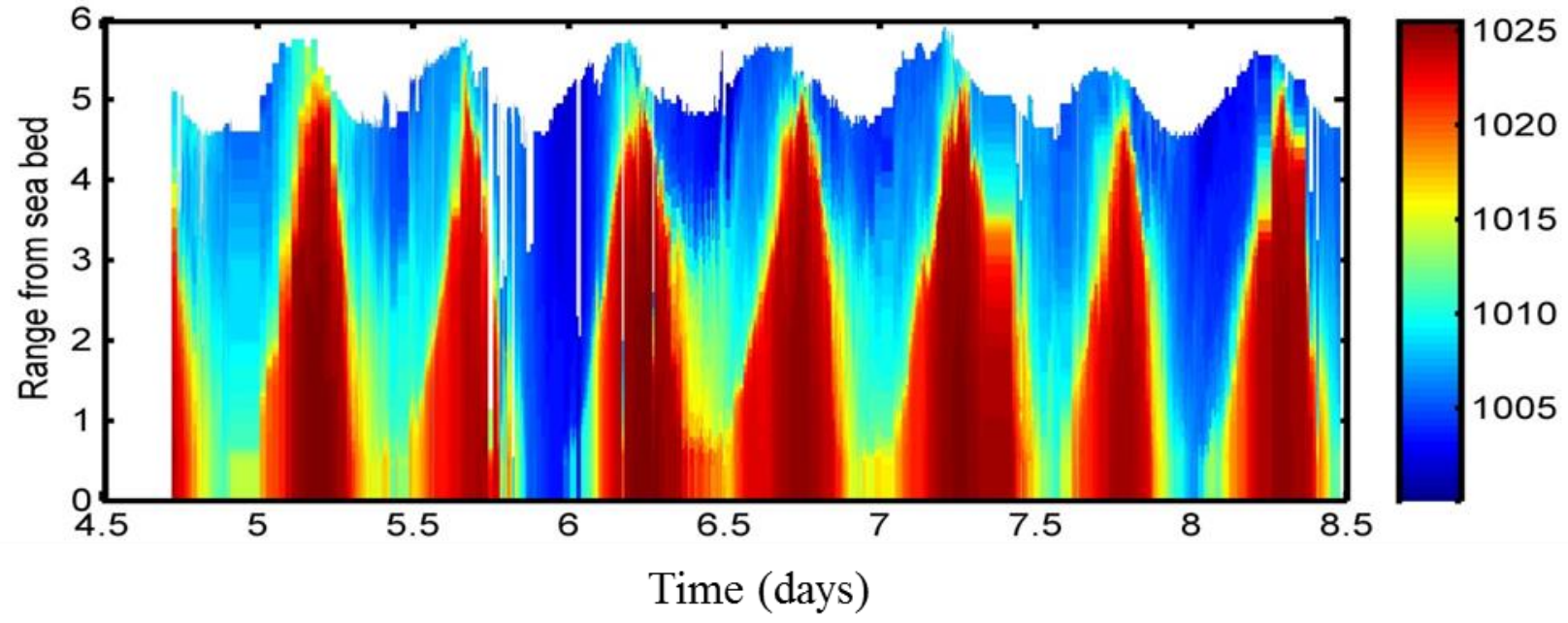

Figure 3A2. Density profiles that were obtained by completing CTD casts for five minutes four times an hour during the experiment. The erosion of the salt wedge during ebb tides and advection back up the river can be seen. Asymmetries in the location of the pycnocline as a function of time between the ebb and flood tides are present. These asymmetries are seen in the velocity profiles in Figure 1A2 as well. The color scale is in $\mathrm{kg} / \mathrm{m}^{3}$.

During the flood tide the velocity near the bed is in the upriver direction as the salt wedge advects up the estuary due to tidal forcing. Nearly an hour into the flood a velocity maxima in the upriver direction develops at the center of the pycnocline as a result of river flow opposing the encroaching salt wedge (Figure 1-3A2). Identifiable shear instabilities occur almost exclusively on the upper edge of the velocity maxima (results not shown). The velocity maxima 
moves higher and higher into the water column as the flood continues until the ebb process begins again as described above (Figure 1A2).

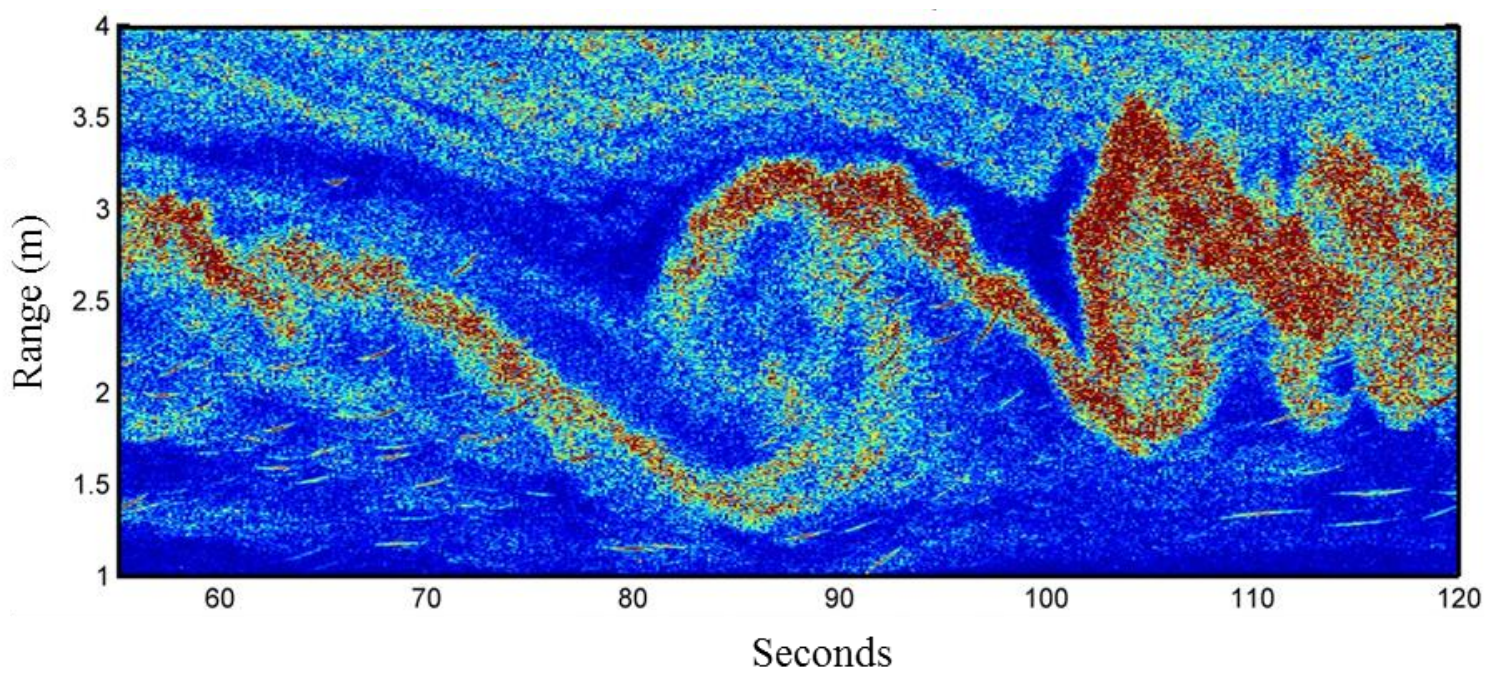

Figure 4A2. Acoustic image from Transducer $4(\mathrm{M})$ at 08:36 on 7 December during the middle of the ebb tide. The $2 \mathrm{~m}$ peak to peak amplitude shear instability is much larger the shear instabilities seen in Observations A through E. It is likely the increase in amplitude due to the broadening of the shear layer. The streaks in the image are most likely fish.

An important aspect of the flow dynamics in Connecticut River, which can be deduced from the velocity profiles is that the shear instabilities are almost always advecting up or down stream depending on the phase of the tide as expected from linear theory [Miles, 1961]. In the Connecticut River the mean velocity of the shear layer is typically non-zero and directed downriver during ebb tides and upriver during flood tides. Therefore, shear instabilities in the Connecticut River translate as they evolve due to the non-zero mean velocity in the shear layer. Thus, measuring the evolution of shear instability in the Connecticut River requires a measurement system capable of tracking shear instabilities, including their amplitude, wavelength and turbulent dissipation as they advect and evolve downstream. 
ADCP measurements: Array perpendicular to flow
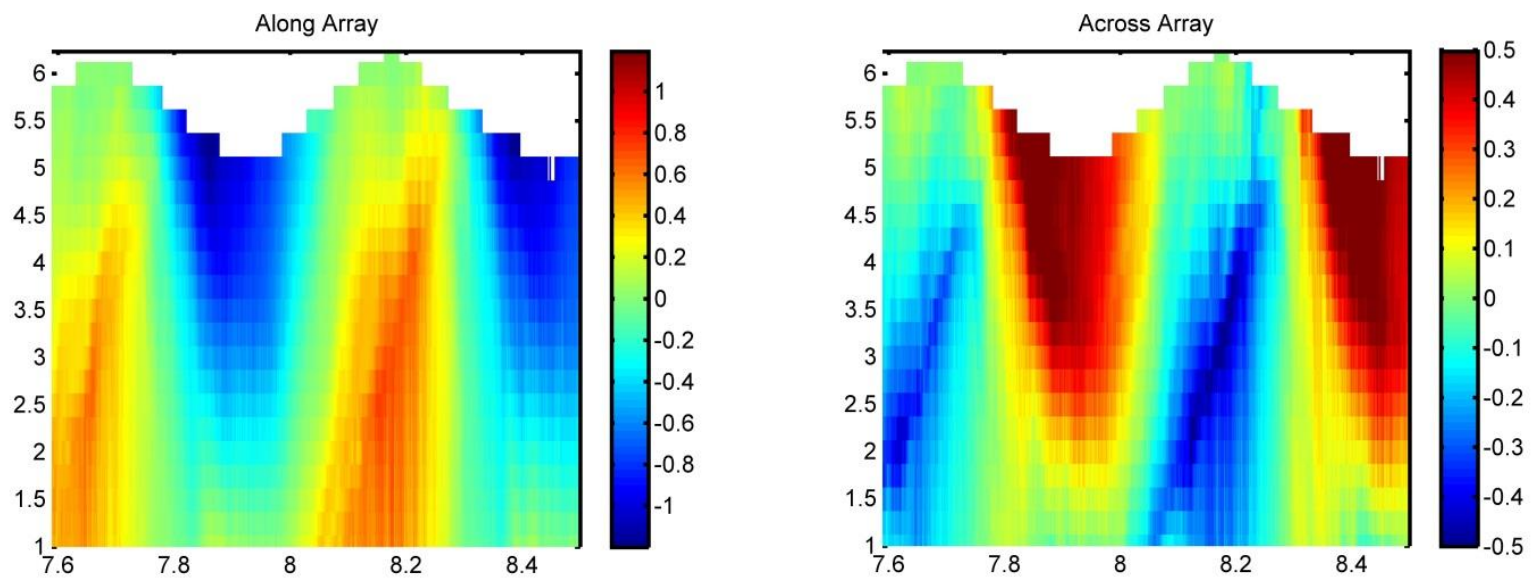

Figure 5A2. ADCP time series collected while the array was perpendicular to the flow. The array was not perfectly aligned so there are velocity components of similar magnitude in the along and cross array directions. Color scale in $\mathrm{m} / \mathrm{s}$.

\section{Contribution of shear instability to mixing}

As part of the analysis of the data set from 5-8 December 2012 the backscatter spectrum and amplitude were calculated throughout the water column for an entire tidal cycle using similar methodology outlined in Lavery et al. [2013]. Instead of using patches of data which consist of $30 \mathrm{~s}$ ping averages averaged over $20 \mathrm{~cm}$ in space as was done in Lavery et al 2013, $1 \mathrm{~s}$ ping averages averaged over $5 \mathrm{~cm}$ in space were used. The shorter spatial and temporal averaging enables estimates of turbulence parameters to be obtained at high resolution compared to more traditional measurement methods. As a result, it is possible to ascertain if turbulence and mixing occur at higher rates when shear instabilities are present. Answering this question could help determine how important shear instabilities are to mixing.

An example calculation is shown from Observation A (Figures 6A2 and 7A2) which suggests turbulence and mixing is significantly higher when shear instabilities are present. A more detailed analysis of this data needs to be done in order to draw any further conclusions, however, these results suggests significant understanding of the role of shear inabilities in the 
Connecticut River could be deduced from a more rigorous and detailed analysis of the acoustic

backscatter data.
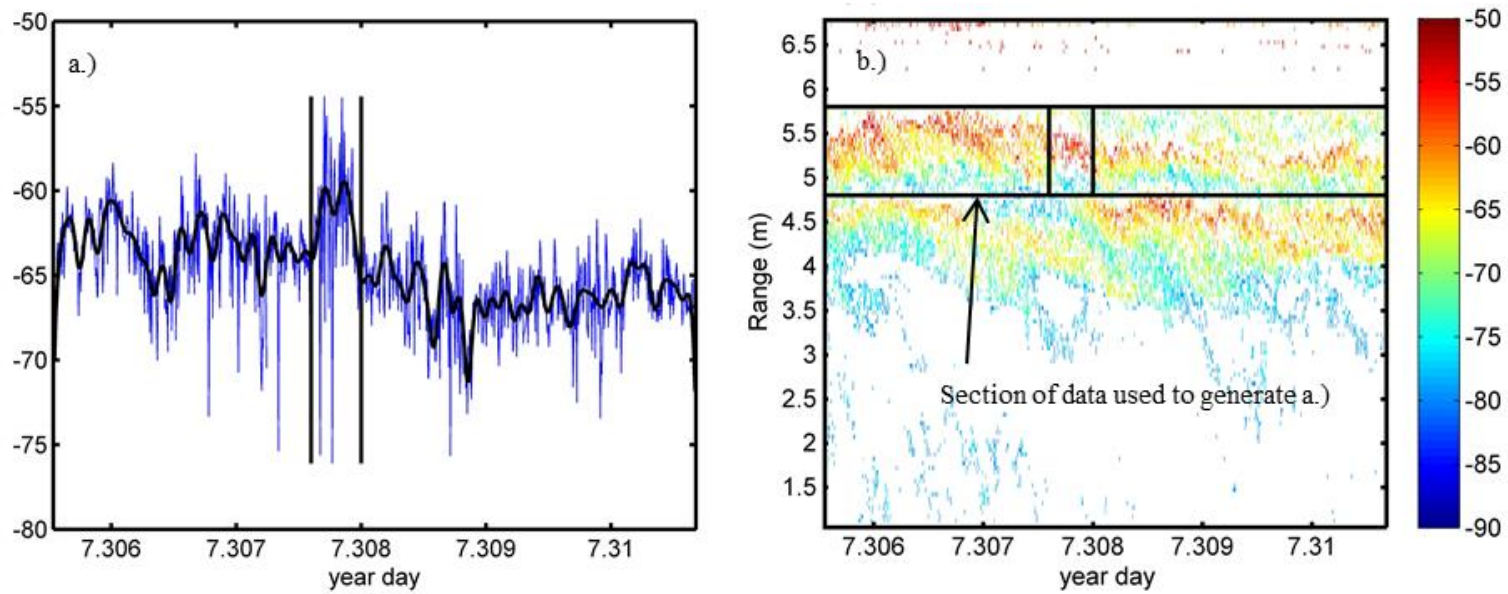

Figure 6A2. a.) Mean volume backscatter in $\mathrm{dB}$ re $1 \mu \mathrm{Pa}$ for a 15 minute section of data estimated from transducers 3 and 4 . Note the large increase in backscatter marked by the vertical black lines. The blue line is the mean volume backscatter through the water column and the black line is the low-passed version of the same data. The vertical black lines are drawn the exact same time in figures 6A2 and7A2 showing that the increase in backscatter is due to the shear instabilities passing by at that time. b) Locations within the water column of scattering consistent with stratified turbulence in the viscous convective sub-range. White regions indicate no turbulence; warm colored regions indicate turbulence and its intensity. The color scale is in $\mathrm{dB}$ re $1 \mu P a$.

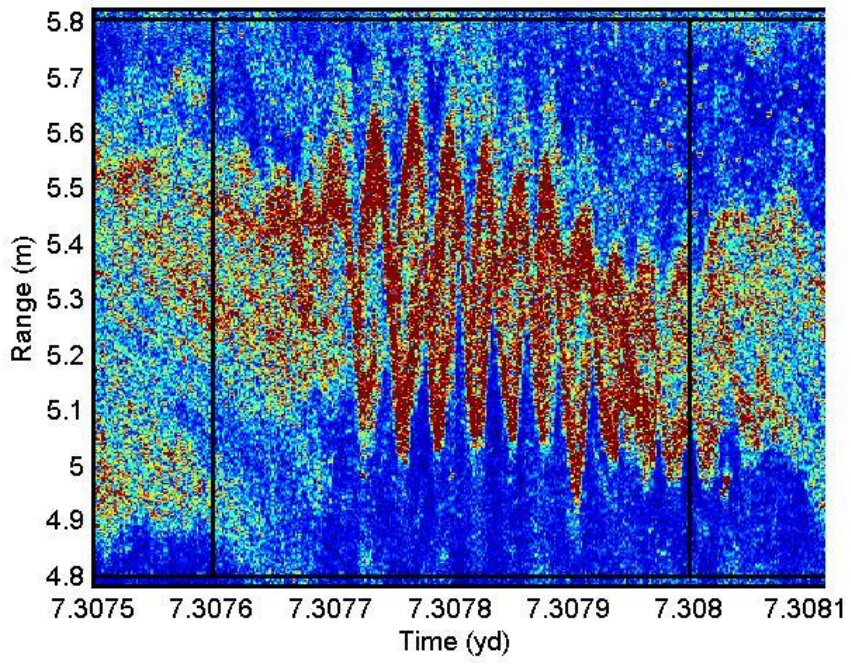

Figure 7A2. Acoustic image from transducer 6 during Observation A. The black lines are drawn at the identical times as in Figure 6A2 a.) and b.), further demonstrating the connection between strong backscatter consistent with stratified turbulence and shear instability. 


\section{Appendix 3: Acoustics theory}

\section{Wave equation for an inhomogeneous medium}

Scattering of sound occurs when an acoustic wave encounters an inhomogeneity in the propagation medium that redirects the incident acoustic waves in many directions. The equation governing the propagation and scattering of acoustic waves is the wave equation,

$$
\nabla \cdot\left(\frac{1}{\rho_{o}} \nabla p\right)-\kappa \frac{\partial^{2} p}{\partial t^{2}}=S
$$

where $\rho_{o}$ is the density of the medium, $p$ is the pressure field, $\kappa$ is the compressibility (related to sound speed and density) and $S$ is a source term. Gradients in density and sound speed directly influence the behavior of an acoustic wave. In the ocean some typical sources of density and sound speed gradients, depending on the frequency of the acoustic waves, are stratified turbulence, gradients of temperature and density associated with mesoscale water masses, fish, zooplankton, sediment and bubbles. The intensity of the acoustic scattering from these sources of inhomogeneity is dependent on the scale of the acoustic wavelength to the scale of the inhomogeneity and the strength of the gradients.

A simple scaling exercise intuitively reveals the frequency dependence of scattering. A typical parameter used to compare the acoustic wavelength (which is related to frequency) to the

scale of the inhomogeneity is $k a$, where $k$ is the acoustic wave number defined as $\frac{2 \pi}{\lambda}$ where $\lambda$ is the acoustic wavelength. $a$ is the characteristic length scale of the inhomogeneity. For $k a \ll 1$ (Figure 1A3) the geometry of a particular inhomogeneity is typically unimportant and the inhomogeneity can be modeled as a point scatterer [Morse and Ingard, 1968]. Additionally, if $k a$ is sufficiently small the scattering from the inhomogeneity may be considered negligible for all practical purposes. For $k a \gg 1$ (Figure 1A3) the geometry of the inhomogeneity becomes 
important as the wavelength of the acoustic waves is on the order of or smaller than the scales of the inhomogeneity [Morse and Ingard, 1968].

$k a \ll 1$

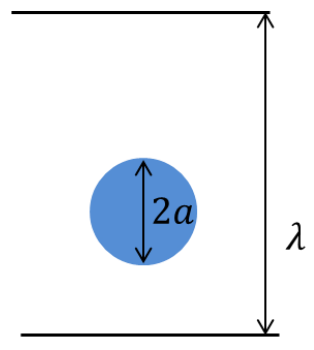

$k a \gg 1$

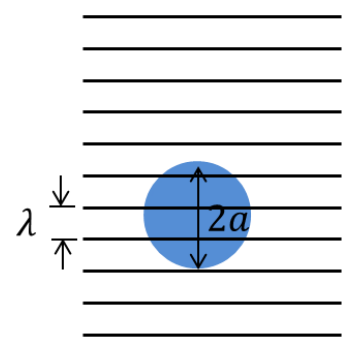

Figure 1A3. Depiction of different values of $k a$ for an arbitrary inhomogeneity. On the left it can be seen the wavelength is much larger than inhomogeneity and on the right the wavelength is much smaller than the inhomogeneity. The inhomogeneity can be thought of as a region of density or sound speed or both that is different from the ambient. Note the scattered fields are not included in this figure.

\section{Broadband Classification}

Using broadband signals, signals which contain a continuous range of frequencies, provides the opportunity to investigate a scatterer or group of scatterers as a function of frequency. The frequency dependence of scattering from inhomogeneities can be used as a means of discriminating between and quantifying scatterers (Figure 2A3). The different spectra that result from different scattering processes can be thought of as acoustic signatures which can be used to identify and quantify the scattering processes (Figure 2A3). Typically ancillary in-situ measurements are needed to aid in this interpretation. For example, in this thesis the CTD and ADCP measurements aid in identify scattering from stratified turbulence. In this thesis frequencies between $160 \mathrm{kHz}$ and $590 \mathrm{kHz}$ are used. Therefore scattering from inhomogeneities on the scales of centimeters to millimeters are of interest like those shown in Figure 2A3.

Broadband techniques for classification of scattering mechanisms in the ocean depend on the development of accurate scattering models. Scattering from the ocean interior can often be assumed to be weak with exception of bubbles and sediment, which can be dominant scattering 
sources depending on location and frequency used. Weak scattering occurs when the amplitude of the scattered acoustic field is much less than the amplitude of the incident acoustic field. When these conditions are met, the Born approximation can be used to predict the scattering strength of a single or multiple scatterers [Lavery et al., 2003].

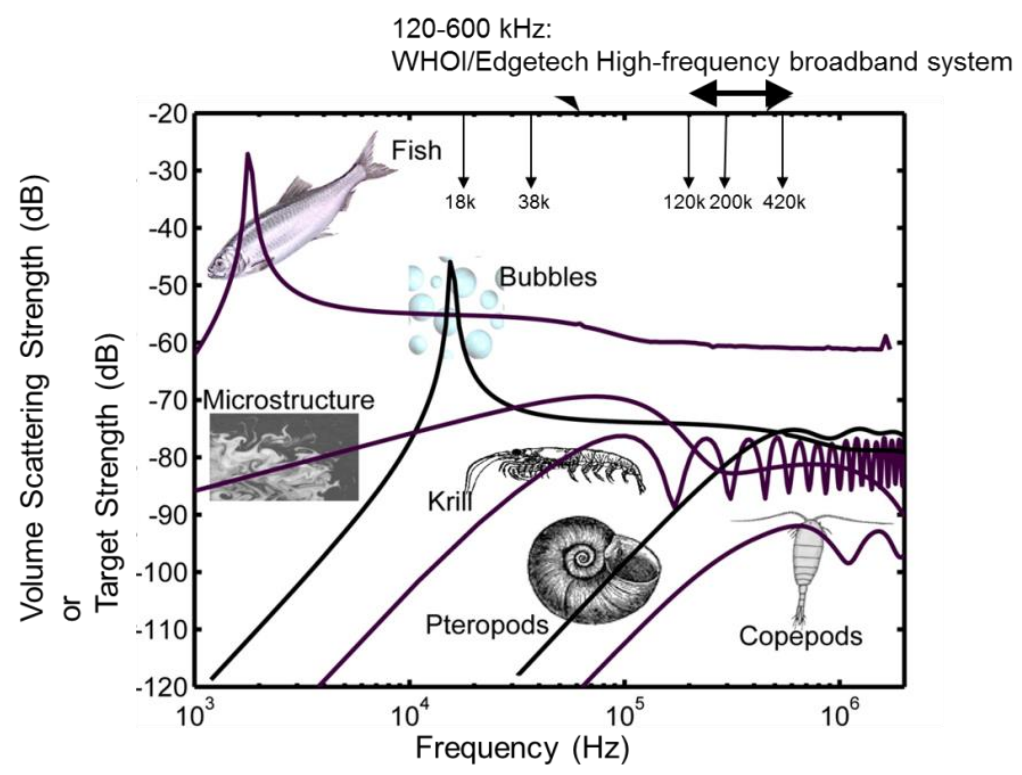

Figure 2A3. Theoretical volume backscatter strength for selected scattering targets in the ocean. Credit: Andone Lavery, Woods Hole Oceanographic Institution, Based on Figure 1 from Lavery et al. [2007].

The Born approximation assumes the incident acoustic wave does not change as it propagates through an inhomogeneity. In general, assuming the solution to the inhomogeneous wave equation is a complex exponential function, the scattered field from any inhomogeneity in the far field due to an incident plane wave is given as

$$
P_{S}=P_{o} \frac{e^{j k r}}{r} \mathrm{f}
$$

where $P_{S}$ is the scattered pressure field, $P_{o}$ is the incident field, $\mathrm{r}$ is the range from the transducer to the scatterer and $\mathrm{f}$ is the scattering efficiency of the inhomogeneity [Ishimaru, 1978]. In the case of stratified turbulence measured using piston transducers, the scattering per unit volume or the volume backscatter cross-section is the parameter that is measured in the field. Therefore to 
classify and quantify stratified turbulence the volume backscatter cross-section must be modeled. Scattering per unit volume is defined as

$$
\sigma_{v}=\frac{r^{2}\left\langle P_{s} P_{s}^{*}\right\rangle}{V P_{o}^{2}}
$$

where $\mathrm{V}$ is the volume of the inhomogeneity and $P_{S}^{*}$ is the complex conjugate of the scattered field [Ishimaru, 1978]. This expression can be further simplified to $\sigma_{v}=\frac{1}{V}\left\langle f f^{*}\right\rangle$ using the Born approximation and using the definition of $\mathrm{f}$

$$
f=\frac{(\pi / 2) k^{4}}{(2 \pi)^{3}} \int_{V} B_{y}(\boldsymbol{r}) e^{-i \boldsymbol{K} \cdot \boldsymbol{r}} d \boldsymbol{r}
$$

where $B_{y}(\boldsymbol{r})$ describes the spatial fluctuations of compressibility and density within the medium, $K$ is the Bragg wave number $\left(=2 k \sin \left(\frac{\theta}{2}\right)\right.$ where $\theta$ is the angle of incidence) and $\boldsymbol{r}$ is the unit vector normal to the surface of the volume [Ishimaru, 1978]. Seawater compressibility and density fluctuations are related to density and sound speed fluctuations in the ocean which are in tern related to temperature and salinity. By assuming $B_{y}(\boldsymbol{r})$ for stratified turbulence in the ocean can be modeled by a one dimensional Bachelor spectrum for salinity and temperature, an analytical expression for $\sigma_{V}$ can be obtained. For the frequency ranges used, the viscous convective sub range of the spectrum is used (see Appendix 4 for more details about the Batchelor spectrum). Using these assumptions the analytical expression for $\sigma_{V}^{v c}$ becomes

$$
\sigma_{V}^{v c}=-\frac{k^{4}}{K}\left(A^{2} \frac{d \phi_{T}(K)}{d k}+B^{2} \frac{d \phi_{S}(K)}{d k}+2 A B \frac{d \phi_{T-S}(K)}{d k}\right)
$$

Where $\phi_{S}$ and $\phi_{T}$ are the one dimensional Batchelor spectrum for salinity and temperature respectively, $\phi_{T-S}$ is the co-spectrum of salinity and temperature, A and B are defined as follows [Lavery et al., 2013 ; Bachelor, 1959]. 


$$
\begin{aligned}
& \mathrm{A}=\frac{1}{C} \frac{\partial C}{\partial T}-\alpha \\
& \mathrm{B}=\frac{1}{\rho} \frac{\partial \rho}{\partial S}-\beta
\end{aligned}
$$

Eq. 7A3

where $\beta$ is the coefficient of saline contraction and $\alpha$ is thermal expansion coefficient [Lavery et al., 2013]. By further assuming temperature and salinity are linearly related and diffuse at the same rate, $\chi_{T}$, the temperature variance can be related to $\chi_{S}$ the salinity variance through the expression $\chi_{T}=\chi_{S}\left(\frac{\partial \bar{T}}{\partial \bar{S}}\right)^{2}$. The purpose for making this assumption is to reduce the number of unknowns to two. This assumption is typically valid in the Connecticut River [Lavery et al., 2013] and this term is also usually quite small in the Connecticut River as there is very little temperature variation in the river in the winter. Using the previously stated assumptions Eq. 5A3, for stratified turbulence in the viscous convective sub-range can be written as

$$
\sigma_{V}^{v c}=2^{-3} \Psi^{a c} q \chi_{s}\left(\frac{\varepsilon}{v}\right)^{-1 / 2} k
$$

where $\Psi^{a c}=A^{2}\left(\frac{\partial \bar{T}}{\partial \bar{S}}\right)^{2}+B^{2}+2 A B\left(\frac{\partial \bar{T}}{\partial \bar{S}}\right)[$ Lavery et al., 2013].

\section{Pulse compression}

The pulse compression or the matched filter is a widely used signal processing technique in radar and acoustic applications. Chu and Stanton [1998] first applied pulse compression methods to oceanographic high frequency broadband acoustic backscatter. In the context of high frequency broadband acoustic backscatter techniques pulse compression is used to enhance the vertical range resolution of the acoustic backscatter recorded within a ping.

Pulse compression is the convolution of the transmitted signal from the transducer with the recorded data from a ping. The wide-band transmit signals result in backscatter signal which 
contains nearly identical bandwidth. The convolution of the transmitted and the received signal results in enhanced range resolution in space due to the nearly identical bandwidth of the received and transmitted signal (Figure 3A3).

a.)

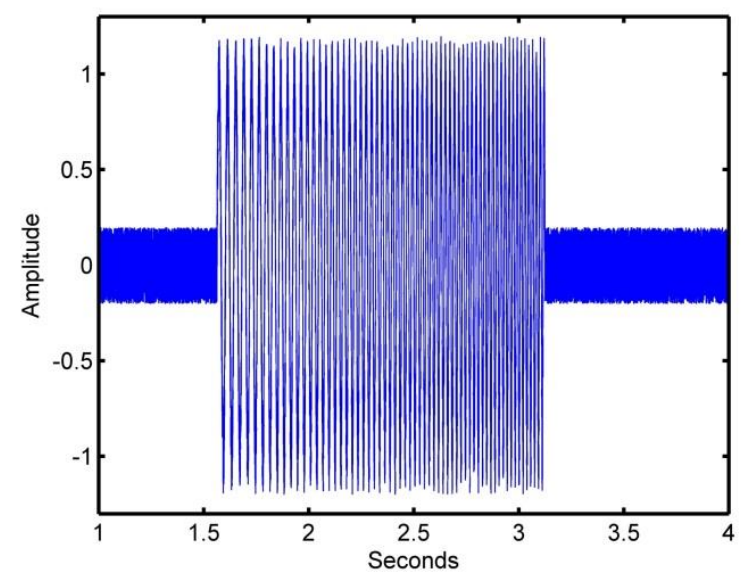

c.)

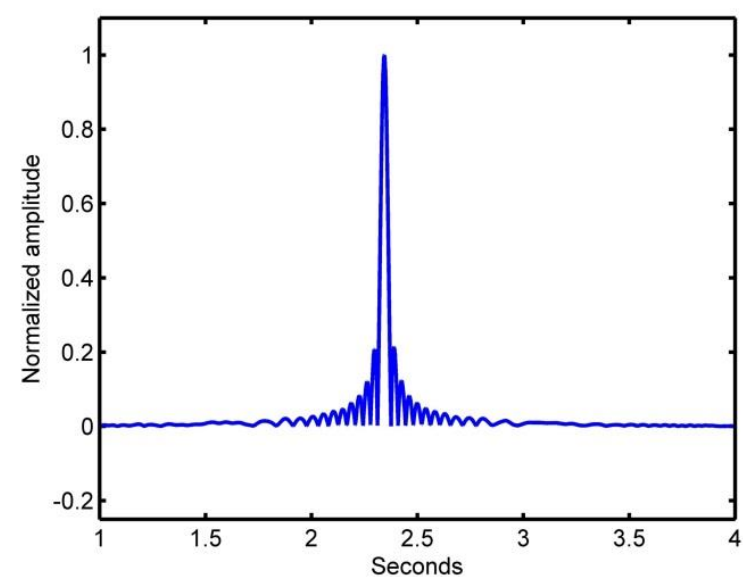

b.

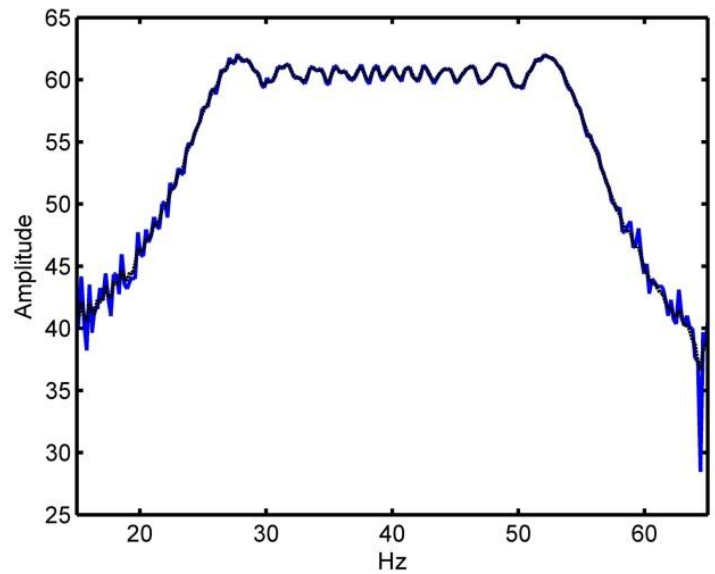

Figure 3A3. a) Chirp signal in the time domain. b.) Discrete time Fourier Transform of the chirp signal (blue) and the pulse compressed chirp signal (blue). c.) pulse compressed chirp signal. Note that figures a and $\mathrm{c}$ are on the same time axis. The range resolution of the signal in figure $\mathrm{c}$ is the inverse bandwidth of the original chirp signal.

The pulse compression operation can be expressed as

$$
P C_{\text {output }}=V_{\text {rcc }}(\mathrm{t}) \otimes V_{\text {chirp }}(\mathrm{t})
$$

where $P C_{\text {output }}$ is the pulse compression output, $V_{\text {rec }}(\mathrm{t})$ is the received signal and $V_{\text {chirp }}(\mathrm{t})$ is the ideal transmit signal and $\otimes$ is the convolution operation. It can be shown analytically that for two signals of identical bandwidth $P C_{\text {output }}$ is equal to a sinc function with a width equal to the inverse bandwidth of the signals [Chu and Stanton, 1998]. 


\section{Calibration}

A requirement for remotely quantifying acoustic backscatter processes in the ocean using spectral methods is having a calibrated acoustic backscatter system. An acoustic system consists of an electronics can that contains the hardware to transmit and digitally record signals on multiple channels. Each channel has a dedicated transducer. Each transducer and channel pair must be individually calibrated to make quantitative estimates of volume backscatter strength.

The calibration procedure employed here broadly followed Lavery et al. [2010]. Each transducer and channel pair on the system are calibrated by placing the entire system in a tank and recording a number of pings with a tungsten carbide sphere (a ball bearing) in the center of the transducer beam for each transducer and channel pair. The tank was of sufficient size such that that neither the ball bearing nor the transducers are in the near field and the backscattered signals did not saturate the digital to analog converter for the channel. Additionally, it is critical that the sphere is in the center of the transducer's beam. Saturation of the scattered signal from or partial ensonification of the calibration sphere will result in significant calibration errors. The tungsten carbide sphere is used for calibration because the exact solution for scattering from an elastic sphere with uniform material properties, such as the calibration sphere, is known. Therefore a ping which contains the backscatter signal from a calibration sphere that is located in the center of the transducer beam can be used to characterize the channel and transducer pair response. The response is obtained by correcting a range gated part of the calibration ping, which contains the backscatter signal from the calibration sphere, for attenuation, beam volume and range effects. The difference between the measured backscatter and the theoretical curve accounts for the system response. Applying the calibrations to field data to obtain calibrated volume backscatter $s_{v}(\omega)$ is done by using the flowing equation from Lavery et al. [2010]. 


$$
S_{v}(\omega)=\frac{\left\langle\left|V_{v o l}^{F F T}(\omega)\right|^{2}\right\rangle}{\left\langle\left|V_{c a l}^{F F T}(\omega)\right|^{2}\right\rangle}\left|\frac{L_{c a l}(\omega)}{L_{v o l}(\omega)}\right|^{2} \frac{\sigma_{b s}^{W C 20}(\omega)}{V(\omega)}
$$

where the following terms are described below.

$$
\begin{array}{cl}
\left\langle\left|V_{v o l}^{F F T}(\omega)\right|^{2}\right\rangle & \text { incoherent average of FFT of field data } \\
\left\langle\left|V_{c a l}^{F F T}(\omega)\right|^{2}\right\rangle & \text { incoherent average of FFT of backscatter from sphere } \\
\left|\frac{L_{c a l}(\omega)}{L_{v o l}(\omega)}\right|^{2}=\frac{r_{v o l}^{2}}{r_{c a l}^{2}} 10^{2 \alpha\left(r_{v o l}-r_{c a l}\right) / 20} & \begin{array}{l}
\text { Correction for range to target, range to calibration sphere and } \\
\text { attenuation }
\end{array} \\
V(\omega)=\frac{1}{2} c T r_{v o l}^{2} \psi_{D}(\omega) & \text { Correction for transducer beam volume } \\
\sigma_{b s}^{W C 20}(\omega) & \text { Theoretical backscattering cross section of the calibration sphere }
\end{array}
$$

Note: $\alpha$ is attenuation in $\mathrm{dB} / \mathrm{m}, r$ is range in $\mathrm{m}, c$ is soundspeed in $\mathrm{m} / \mathrm{s}, T$ is the length of the time window selected from a ping in seconds and $\psi_{D}(\omega)$ is the frequency dependent beam width of the transducer used Lavery et al. (2010). 


\title{
Appendix 4: Stratified flow and turbulence theory
}

\author{
Stratified shear instability
}

Stratified shear instability within a stably stratified environment (Figure 1A4) occur when the shear becomes sufficiently strong compared to the stratification in the flow. This balance is parameterized by the Richardson number $(R i)$. It has been theoretically proven [Miles, 1961] and empirically confirmed that $R i<0.25$ is a sufficient condition for instability to occur and has been subsequently validated in field and laboratory experiments [Thorpe, 1973; Koop and Browand; 1979; Geyer and Smith, 1987]. The theoretical prediction first made by Miles (1961) was done using the Taylor-Goldstein equation.

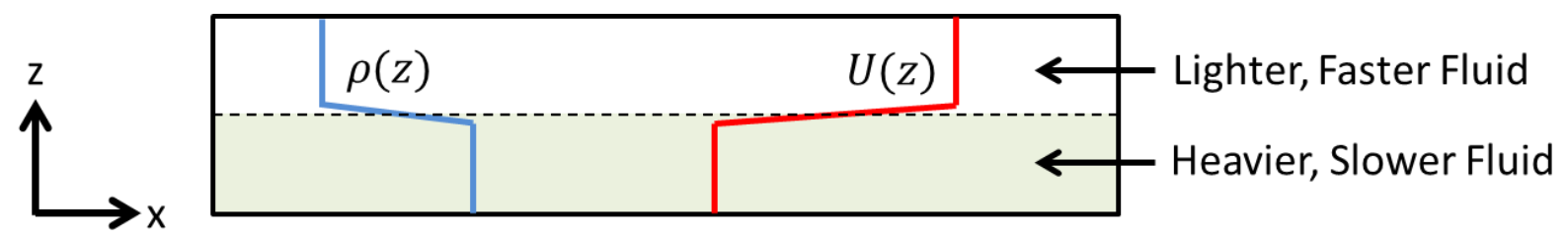

Figure 1A4. A stably stratified and sheared environment illustrating the basic flow characteristics in the Connecticut River. When the shear is large enough compared to the stratification the heavier fluid can be lifted on top of the lighter fluid via a growing wave, initiating turbulent mixing.

The Taylor-Goldstein equation is a linearized, 2D equation derived from Navier-Stokes equations, with viscous and many non-linear terms thrown out through scaling arguments. The equation is used to calculate theoretical stability constraints and growth rates of shear instabilities [Miles, 1961; Hazel, 1972]. The equation, as given by Hazel (1972) is

$$
\frac{d^{2} w}{d z^{2}}+\left[\frac{N^{2}(z)}{(U(z)-C)^{2}}-\frac{d^{2} U / d z^{2}}{U(z)-C}-k^{2}\right]=0
$$

where $w$ is the vertical perturbation velocity, $\mathrm{U}$ is the horizontal mean background flow, $C$ is the phase speed of the perturbation, $\mathrm{k}$ is the wave number of the perturbation and, $N^{2}=$ $\frac{-g}{\rho} \frac{\partial \rho}{\partial z}$. Solutions to this differential equation with a prescribed velocity and density profile is 
found by solving an eigenvalue problem for $C$. Un-stable solutions involve values of $C$ that have real and imaginary parts. A solution that contains a phase speed with an imaginary part is unstable. The imaginary part of $C$ is the exponential growth rate of a particular wavelength mode that satisfies the Taylor-Goldstein equation.

Hazel (1972) numerically investigated the growth rates for different idealized velocity and density profiles. The results of Hazel's 1972 study has allowed some simple scaling relationships to be established between the shear layer thickness $(\Delta z)$ and the wavelength $(\lambda)$ of the wave which grows the fastest from the instability from idealized profiles. Hazel (1972) found that the fastest growing mode for hyperbolic tangent density and velocity profiles with identical thicknesses has a non-dimensional wavelength $(\alpha)$ of approximately 0.48 where

$$
\alpha=k \Delta z / 2
$$

It can now be seen the interface thickness of a given wavelength wave that has gone unstable is

$$
\Delta z=2 * 0.48 / k
$$

This scaling relationship is useful for estimating the expected wavelengths of waves growing from shear instabilities in a given environment.

\section{Energy and scalar variance spectrums in stratified and turbulent flow}

The velocity and scalar fields within a turbulent flow are commonly described in terms of statistical distributions which can be expressed in terms of a spectrum. Statistical descriptions are used because turbulent flows contain many scales of motion making it difficult to gain analytical insight into the flows any other way. In the context of this thesis, analytical models that describe the turbulence and passive scalars within the turbulent flows are needed to acoustically invert for turbulence parameters such as $\varepsilon$. It should be noted that backscatter is only sensitive to sound 
speed and density gradients not to fluid velocity fluctuations. However, the knowledge of velocity fluctuations are needed to model density and sound speed variations within a turbulent medium. In terms of describing the energy or velocity fluctuations in a turbulent flow, the Kolmogorov spectrum predicts the rate of turbulent kinetic energy dissipation for homogeneous and isotropic turbulence. In terms of describing the passive scalars within a turbulent flow, the Batchelor spectrum is used, which also assumes homogeneous and isotropic turbulence.

The Kolmogorov spectrum describes the energy dissipation for the range of scales that occupy the inertial subrange. The inertial subrange is defined as the range of wave numbers ( $k=2 \pi / l$, where 1 is a length scale) where homogeneous isotropic turbulence is expected to exist. More specifically, the inertial subrange contains the scales smaller than the largest possible eddy and larger than the scales where viscous diffusion smears out velocity gradients. The range of wave numbers is defined as $\mathrm{K}<<\mathrm{k}<<2 \pi \eta^{-1}$ where

$$
\begin{array}{ll}
\eta=\left(v^{3} / \varepsilon\right)^{1 / 4} & \text { Eq. } 4 \mathrm{~A} 4 \\
K=2 \pi / L_{o} & \text { Eq. } 5 \mathrm{~A} 4
\end{array}
$$

and $v$ is the kinematic viscosity of the fluid, $\varepsilon$ is the turbulent kinetic energy dissipation rate and $L_{o}$ represents the largest scale eddy in the flow. $\eta$ is often referred to the Kolmogorov microscale and is the scale at which viscosity begins to dissipate energy in the flow and relax velocity gradients. $K$ represents the largest scale turbulent eddies in the flow. The energy spectrum in the inertial subrange is given by

$$
E(k)=c_{0} \varepsilon^{2 / 3} k^{-5 / 3}
$$

where is $c_{0}$ the Kolmogorov constant and has a value of approximately 0.5 [Sreenivasan, 1995]. The range of wave numbers the inertial subrange spans is a function of the Reynolds number. The larger the Reynolds number the greater number of wave numbers the inertial subrange spans 
within the flow. Further, increasing the Reynolds number typically pushes the end of the inertial sub-range to smaller rather than larger scales.

In the case of stratified turbulence $L_{o}$ is the scale at which gravity no longer effects the turbulent eddies. This scale is given by the Ozmidov scale, $L_{O}=\sqrt{\varepsilon / N^{3}}$ where $N=\sqrt{\frac{g}{\bar{\rho}} \frac{\Delta \rho}{\Delta z}}$ [Kundu, 2012]. Therefore, the inertial subrange in stratified flows will only exist for eddies on scales less than $L_{O}$.

The Batchelor spectrum expresses the spectrum of a given passive scalar within in a stratified and turbulent flow. The Batchelor spectrum is valid for turbulence which is homogeneous and isotropic and is directly related to the inertial subrange from the Kolmogorov spectrum. Further, the Prandlt number $\left(\operatorname{Pr}=\frac{v}{\kappa}\right)$ plays an essential role in the structure of the spectrum. There are two sub-ranges of the Batchelor spectrum, the inertial convective and viscous convective subranges (Figure 2A4). For any turbulent flow with $\operatorname{Pr}>1$ for which an inertial subrange exists the inertial convective subrange also exists. The inertial convective subrange is given by

$$
S(k)=c_{0} \chi \varepsilon^{-1 / 3} k^{-5 / 3}
$$

where $\chi$ is the rate of variance dissipation of a passive scalar in the flow in units $^{2} / s$ where units could be ${ }^{\circ} \mathrm{C}$ for example [Batchelor, 1959].

If $\operatorname{Pr} \gg 1$ and $R e$ is sufficiently large then there also exists what is called the viscous convective subrange. The viscous convective subrange includes the scales where the viscosity controls the flow of the fluid, i.e. $k>\eta^{-1}$, but diffusion of the scalar being mixed is still slow 
compared to its advection time and therefore the passive scalar is not yet getting smoothed out by diffusion [Kundu, 2012]. The spectrum is given by

$$
S(k)=c_{0} \chi(\varepsilon / v)^{-1 / 2} k^{-1}
$$

in the viscous convective subrange[Batchelor, 1959].

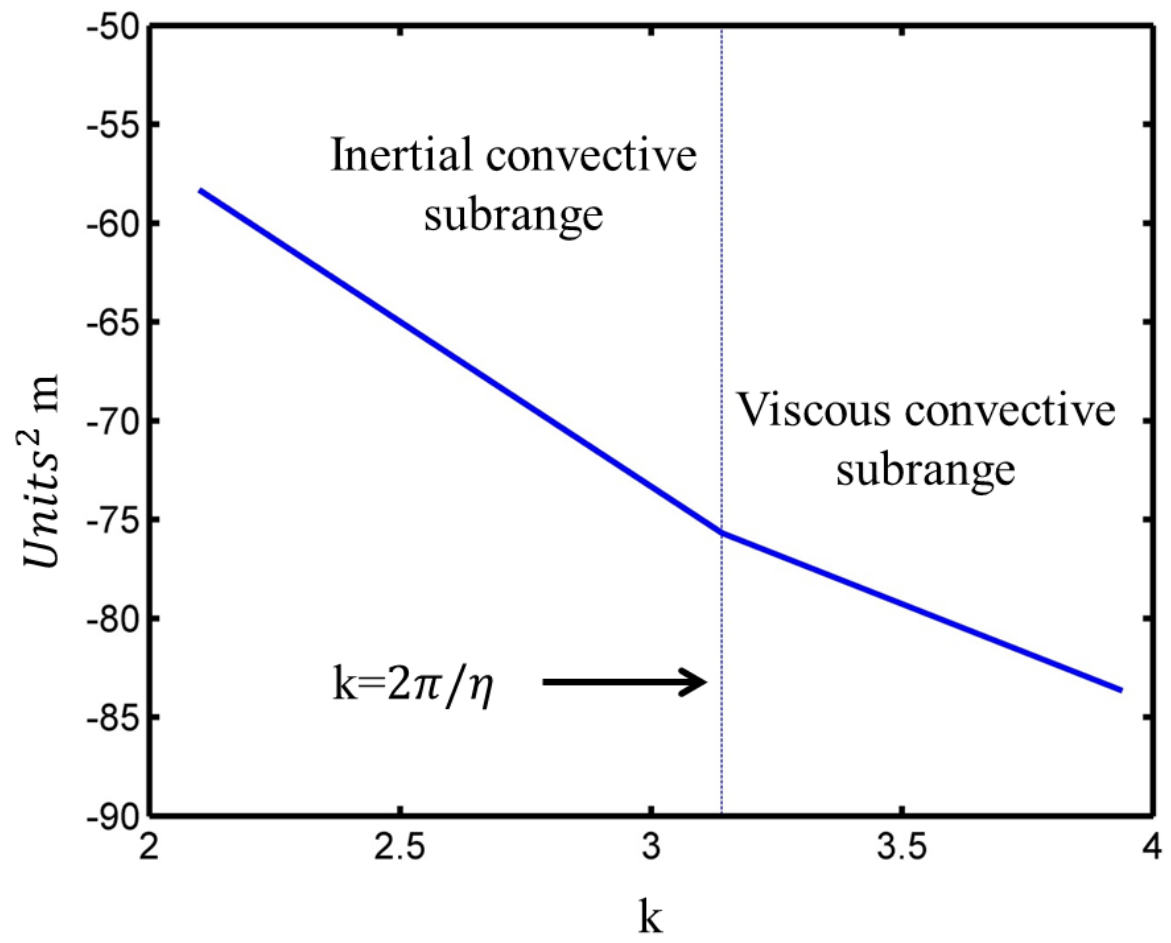

Figure 2A4. A theoretical Batchelor spectrum for a passive tracer such as salinity or temperature in a stratified and turbulent flow. Note the change in slope of the spectrum at the Kolmogorov micro-scale which demarks the transition between the inertial and viscous convective subranges. 


\section{Appendix 5: Array design}

The array was designed with one primary scientific goal and numerous significant engineering constraints. Scientifically the goal was to image the largest range of shear instability wave lengths possible using the six available transducers. The array had to be bottom mounted because the other ships involved with the experiment were unable to carry additional instrumentation or sample in the area of interest. The most significant engineering constraints that affected the science goals consisted of the following:

1. The array needed to fit on the deck of the $60 \mathrm{ft}$. R/V Tioga which was used to deploy the array along with other instrumentation needed for the deployment

2. The array needed to be streamlined and heavy enough such that the flow would not dislodge it from its deployment location

3. The array needed to be tall enough so it would not get buried if a sand wave migrated over it and low enough not to interfere with the flow

4. The acoustic beams from the transducers could not overlap

The R/V Tioga could only carry an array that was $6 \mathrm{~m}$ long so the array length was set by this constraint. In terms of weight and streamlining the array was made out of channel iron (Figure 1A5) which is heavy and thin. Additionally, lead weights were added to the feet as an extra precaution. The array was made $0.75 \mathrm{~m}$ tall as a compromise between it obstructing the flow and keeping it safe from burial (Figure 2A5). From previous observations it was known the largest sand waves in the area were on the order of one meter. To avoid beam width overlap the transducers could not be spaced any closer than $1 \mathrm{~m}$ apart based on the largest transducer full beam width of $10^{\circ}$. 
The impact of the engineering constraints on the scientific goals was significant. Using scaling from Hazel (1972) and previous observations of shear instability in the Connecticut River it was determined waves with wavelengths from 2-20 m were expected to occur during the deployment. It was assumed at least one e-folding time scale was needed to capture a significant portion of shear instability evolution. Assuming the waves would advect with the mean speed of the shear layer they originated from it was estimated 20-30 m long array was needed. In terms of spacing, half wavelength transducer spacing would allow for the most accurate tracking of the shear instabilities downstream. Such spacing would require 20-30 transducers over the length of the desired array. Clearly the ideal array design was not possible given the number of transducers available and the length restriction set by the R/V Tioga. As a result the array could only be used to capture the evolution of the smallest wave length waves growing from shear instabilities in the Connecticut River.

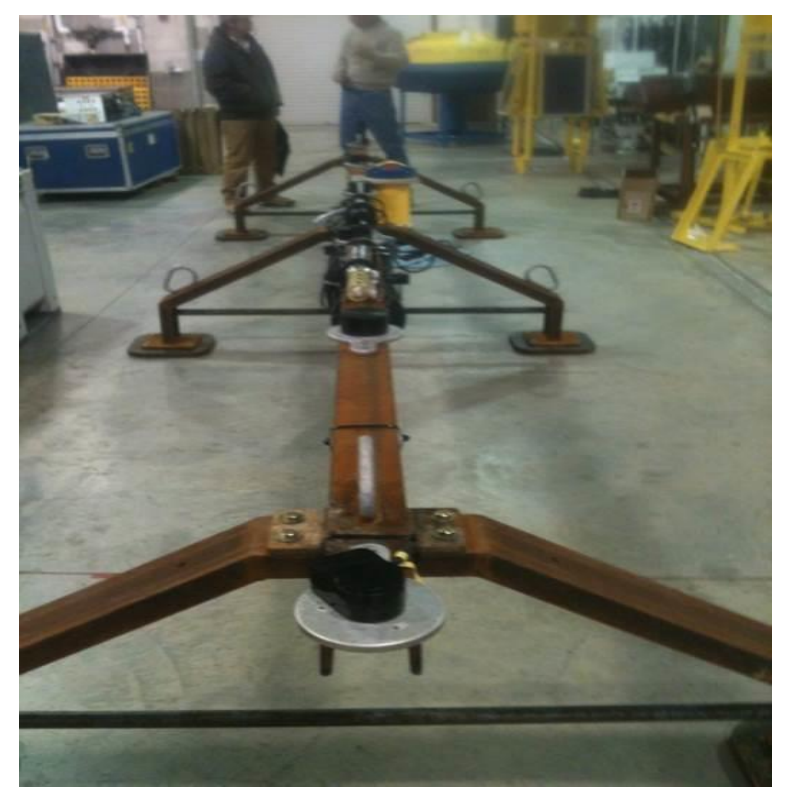

Figure 1A5. The high frequency broadband acoustic backscatter array. Transducers were rigidly secured to the channel iron frame and lead weights were attached to its feet. Photograph credit: Andone Lavery. 


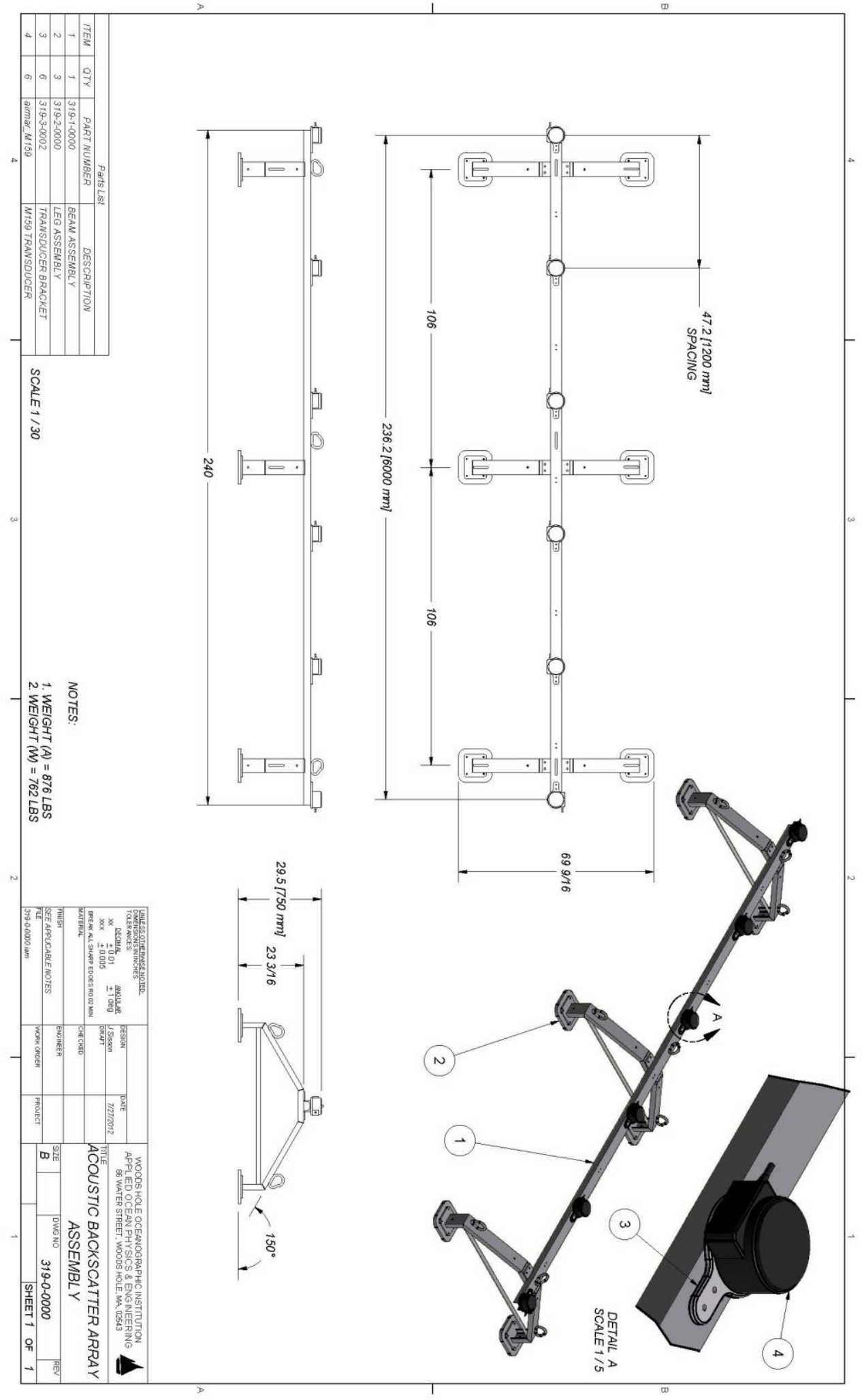

Figure 2A5. The high frequency broadband acoustic backscatter array dimensions. Credit: Jay Sisson, Woods Hole Oceanographic, Senior Engineering Assistant II. 